\title{
Non-Isomorphic Product Systems
}

\author{
Boris Tsirelson
}

\begin{abstract}
Uncountably many mutually non-isomorphic product systems (that is, continuous tensor products of Hilbert spaces) of types $I I_{0}$ and $I I I$ are constructed by probabilistic means (random sets and off-white noises), answering four questions of W. Arveson.
\end{abstract}

\section{Contents}

Introduction 2

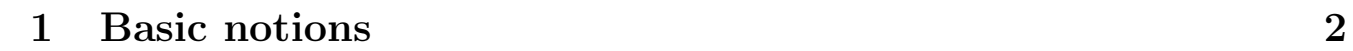

$\begin{array}{lll}2 & \text { Some invariants } & 11\end{array}$

\begin{tabular}{|lll}
3 & Continuous products of measure classes & 19
\end{tabular}

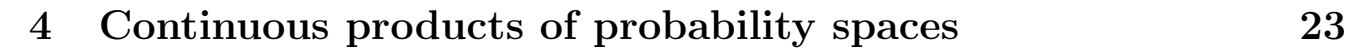

$\begin{array}{lll}5 & \text { Random sets, and type } I I_{0} & 24\end{array}$

$\begin{array}{lll}6 & \text { Constructing random sets } & 27\end{array}$

$\begin{array}{lll}7 & \text { Time reversal } & 36\end{array}$

8 FHS space: logarithm of a Hilbert space 39

9 Continuous sums and off-white noises 45

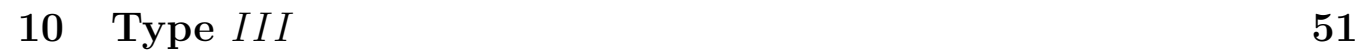

11 The invariant via the logarithm 53

\begin{tabular}{|lll}
\hline 12 & Ensuring asymptotic orthogonality & 58
\end{tabular}

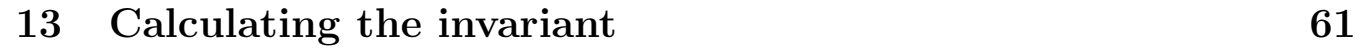

\begin{tabular}{lc}
\hline References & 68
\end{tabular}

\begin{tabular}{lc}
\hline Index & 69
\end{tabular}

\footnotetext{
${ }^{1}$ Supported by the ISRAEL SCIENCE FOUNDATION founded by the Israel Academy of Sciences and Humanities.
} 


\section{Introduction}

It is very difficult to construct examples of types $I I$ and $I I I$.

W. Arveson A99, p. 166]

Product systems appeared in Arveson A89 as a tool for investigating quantum dynamics (namely, $E_{0}$-semigroups). Fruitful interplay between continuous (tensor) products of various spaces, started in Tsirelson and Vershik TV98, is crucial for the present work. For Hilbert spaces, the idea goes back to Araki and Woods [AW66]; for probability spaces — to Feldman [Fe71]; measure classes? appeared in [TV98. Also, the word 'continuous' in 'continuous product' may refer to various bases, not just $[0, \infty)$. Especially, complete Boolean algebras are used in AW66 and [Fe71]. Relations between product systems and continuous products are clarified in Sect. 1.

Main results: Existence of a continuum of mutually non-isomorphic product systems of type $I I_{0}$ (Theorem 6.13), both symmetric and asymmetric (Theorems 7.1, 7.3), and of type III (Theorem 13.11). Four questions asked by Arveson [A89], [A96], [A97], A99 are thus answered.

Main ingredients: Anatoly Vershik's idea of a continuous product of measure classes; an idea of Jonathan Warren (private communication, Nov. 1999) of constructing a continuous product of measure classes out of a given random set; and the author's ideas about appropriate invariants (Sect. 2), random sets (Sect. 5-7), FHS spaces and off-white noises (sect. 8-13).

\section{AbBreviations}

CP Continuous product... HS ... of Hilbert spaces

CPs Continuous products... MC ... of measure classes

HCP Homogeneous continuous product... PS ... of probability spaces

Thus, 'HCPs HS' means: homogeneous continuous products of Hilbert spaces.

\section{Basic notions}

An oversimplified caricature of our objects is the Hilbert space $H=$ $L_{2}\left(\{-1,+1\}^{\mathbb{Z}}\right)$ of all square integrable measurable functions on the probability space $\{-1,+1\}^{\mathbb{Z}}$ of all two-sided sequences of \pm 1 , the product of $\mathbb{Z}$ copies of $\{-1,+1\}$, both -1 and +1 being of probability $1 / 2$. Relevant structures on $H$ are the shift operation $\theta: H \rightarrow H$ and decompositions $H=H_{-\infty, \infty}=H_{-\infty, t-1} \otimes H_{t, \infty}$, where $H_{s, t}=L_{2}\left(\{-1,+1\}^{\mathbb{Z} \cap[s, t]}\right)$. Shifts $\theta^{u}$

\footnotetext{
${ }^{2}$ Called 'measure-type spaces'; the term 'measure class' is borrowed from Arveson.
} 
tie together these decompositions by sending $H_{-\infty, t-1}$ to $H_{-\infty, t+u-1}$ and $H_{t, \infty}$ to $H_{t+u, \infty}$. The caricature uses discrete time $(\mathbb{Z})$, while our true objects use continuous time $(\mathbb{R})$, which leads to a deeper theory.

Throughout, either by assumption or by construction, all Hilbert spaces will be separable. []

We consider a one-parameter group of unitary operators on the tensor product of two Hilbert spaces $H_{-}$and $H_{+}$(denoted also $H_{-\infty, 0}$ and $H_{0, \infty}$ ) and corresponding automorphisms $\alpha_{t}$ of the operator algebra $\mathcal{B}\left(H_{-} \otimes H_{+}\right)$,

$$
\alpha_{t}(A)=e^{i t X} A e^{-i t X}, \quad A \in \mathcal{B}\left(H_{-} \otimes H_{+}\right), \quad t \in \mathbb{R},
$$

satisfying two equivalent conditions:

$$
\begin{array}{ll}
\alpha_{t}\left(\mathcal{B}\left(H_{-}\right) \otimes \mathbf{1}\right) \subset \mathcal{B}\left(H_{-}\right) \otimes \mathbf{1} & \text { for } t \leq 0 ; \\
\alpha_{t}\left(\mathbf{1} \otimes \mathcal{B}\left(H_{+}\right)\right) \subset \mathbf{1} \otimes \mathcal{B}\left(H_{+}\right) & \text {for } t \geq 0 ;
\end{array}
$$

here $\mathcal{B}\left(H_{-}\right) \otimes \mathbf{1}$ is the algebra (in fact, type $I_{\infty}$ factor) of all operators $A \otimes \mathbf{1}$ on $H_{-} \otimes H_{+}$, where $A \in \mathcal{B}\left(H_{-}\right)$and $\mathbf{1}$ is the identity operator on $H_{+}$.

For each $t$,

$$
\mathcal{A}_{-\infty, \infty}=\mathcal{A}_{-\infty, t} \otimes \mathcal{A}_{t, \infty}
$$

where

$$
\begin{gathered}
\mathcal{A}_{-\infty, t}=\alpha_{t}\left(\mathcal{B}\left(H_{-}\right) \otimes \mathbf{1}\right), \quad \mathcal{A}_{t, \infty}=\alpha_{t}\left(\mathbf{1} \otimes \mathcal{B}\left(H_{-}\right)\right) \\
\mathcal{A}_{-\infty, s} \subset \mathcal{A}_{-\infty, t} \text { and } \mathcal{A}_{s, \infty} \supset \mathcal{A}_{t, \infty} \text { for } s \leq t
\end{gathered}
$$

by (1.4) we mean that $\mathcal{A}_{-\infty, t}$ and $\mathcal{A}_{t, \infty}$ mutually commute and generate $\mathcal{A}_{-\infty, \infty}$ as a von Neumann algebra. Moreover, introducing

$$
\mathcal{A}_{s, t}=\mathcal{A}_{-\infty, t} \cap \mathcal{A}_{s, \infty}
$$

we get

$$
\mathcal{A}_{r, t}=\mathcal{A}_{r, s} \otimes \mathcal{A}_{s, t} \quad \text { for }-\infty<r<s<t<\infty,
$$

see $[\mathrm{A},(1.2)]$. If, in addition, both intersections $\cap_{t} \mathcal{A}_{-\infty, t}$ and $\cap_{t} \mathcal{A}_{t, \infty}$ are trivial (that is, equal $\mathbb{C} \cdot \mathbf{1})$, then the pair $\left(\left(e^{i t X}\right)_{t \in \mathbb{R}}, \mathcal{B}\left(H_{-}\right) \otimes \mathbf{1}\right)$ is a history as defined by Arveson [A, Sect. 1.2].

\footnotetext{
${ }^{3}$ For now, they are complex (that is, over $\mathbb{C}$ ), but in Sect. 2 we will return to the point.
} 
Endomorphisms $\alpha_{t}^{-}: \mathcal{B}\left(H_{-}\right) \rightarrow \mathcal{B}\left(H_{-}\right), \alpha_{t}^{+}: \mathcal{B}\left(H_{+}\right) \rightarrow \mathcal{B}\left(H_{+}\right)$defined for $t \geq 0$ by

$$
\begin{gathered}
\alpha_{-t}(A \otimes \mathbf{1})=\alpha_{t}^{-}(A) \otimes \mathbf{1} \quad \text { for } A \in \mathcal{B}\left(H_{-}\right), \\
\alpha_{t}(\mathbf{1} \otimes A)=\mathbf{1} \otimes \alpha_{t}^{+}(A) \quad \text { for } A \in \mathcal{B}\left(H_{+}\right),
\end{gathered}
$$

form so-called $E_{0}$-semigroups $\alpha^{-}, \alpha^{+}$, see [A], Sect. 1.2], irrespective of the additional condition of tail triviality (the latter means that the $E_{0}$-semigroups are pure).

It is well-known (see $\mathrm{A}$, Sect. 3.1]) that each $E_{0}$-semigroup (pure or not) leads to a product system (of Hilbert spaces). Namely, for $t>0$, the set $\mathcal{E}(t)=\left\{T \in \mathcal{B}\left(H_{+}\right): \forall A \in \mathcal{B}\left(H_{+}\right) \alpha_{t}^{+}(A) T=T A\right\}$ is actually a Hilbert space, and multiplication acts like tensoring in the sense that $S T=$ $W_{s, t}(S \otimes T)$ for some (unique) unitary operator $W_{s, t}: \mathcal{E}(s) \otimes \mathcal{E}(t) \rightarrow \mathcal{E}(s+t)$.

On the other hand, our objects are a bit more structured than just histories; the decomposition of our Hilbert space into $H_{-} \otimes H_{+}$is only an example. The general decomposition is, roughly speaking,

$$
H_{-\infty, \infty}=H_{-\infty, t_{1}} \otimes H_{t_{1}, t_{2}} \otimes \ldots \otimes H_{t_{n-1}, t_{n}} \otimes H_{t_{n}, \infty}
$$

for $-\infty<t_{1}<\cdots<t_{n}<\infty$. Here is a rigorous definition.

1.5 Definition. (a) A continuous product of Hilbert spaces ('CP HS', for short $)$ is $\left(\left(H_{s, t}\right)_{-\infty \leq s<t \leq \infty},\left(W_{r, s, t}\right)_{-\infty \leq r<s<t \leq \infty}\right)$ where each $H_{s, t}$ is a Hilbert space, $\operatorname{dim} H_{s, t}>0$, and each $W_{r, s, t}: H_{r, s} \otimes H_{s, t} \rightarrow H_{r, t}$ is a unitary operator, satisfying the condition

(a1) [associativity] the diagram

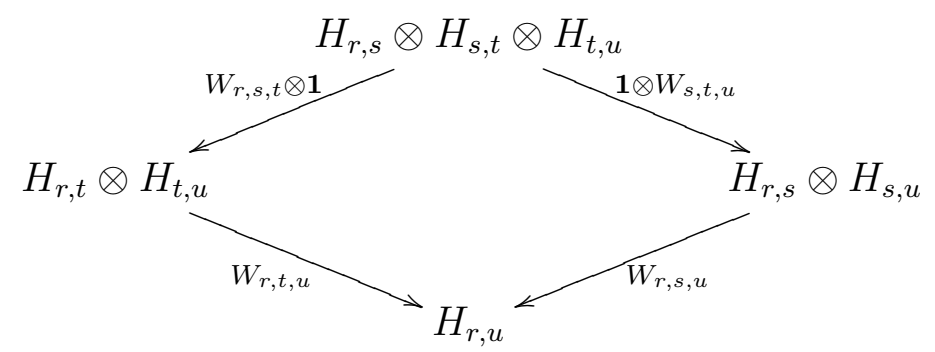

is commutative whenever $-\infty \leq r<s<t<u \leq \infty$.

The CP HS is tail trivial, if it satisfies the condition (a2) [tail triviality]:

$$
\bigcap_{t \in \mathbb{R}} \mathcal{A}_{-\infty, t}=\mathbb{C} \cdot \mathbf{1}=\bigcap_{t \in \mathbb{R}} \mathcal{A}_{t, \infty},
$$


where $\mathcal{A}_{-\infty, t}=\left\{W_{-\infty, t, \infty}(A \otimes \mathbf{1}) W_{-\infty, t, \infty}^{-1}: A \in \mathcal{B}\left(H_{-\infty, t}\right)\right\}, \mathcal{A}_{t, \infty}=$ $\left\{W_{-\infty, t, \infty}(\mathbf{1} \otimes A) W_{-\infty, t, \infty}^{-1}: A \in \mathcal{B}\left(H_{t, \infty}\right)\right\}$.

(b) An isomorphism of one CP HS $\left(\left(H_{s, t}\right)_{s<t},\left(W_{r, s, t}\right)_{r<s<t}\right)$ to another CP HS $\left(\left(H_{s, t}^{\prime}\right)_{s<t},\left(W_{r, s, t}^{\prime}\right)_{r<s<t}\right)$ is a family $\left(U_{s, t}\right)_{-\infty \leq s<t \leq \infty}$ of unitary operators $U_{s, t}: H_{s, t} \rightarrow H_{s, t}^{\prime}$ such that the diagram

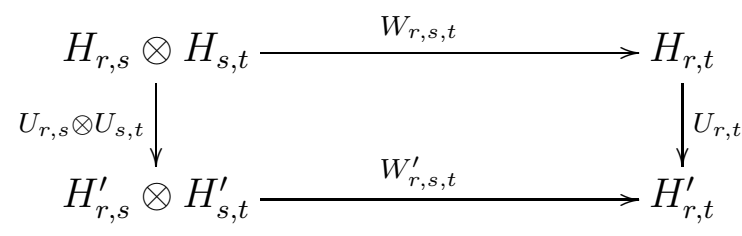

is commutative whenever $-\infty \leq r<s<t \leq \infty$.

Given a CP HS $\left(\left(H_{s, t}\right)_{s<t},\left(W_{r, s, t}\right)_{r<s<t}\right)$ and $u \in \mathbb{R}$, the object $\left(\left(H_{s+u, t+u}\right)_{s<t},\left(W_{r+u, s+u, t+u}\right)_{r<s<t}\right)$ is also a CP HS; an isomorphism $\theta^{u}$ from the former to the latter may be called a shift (by $u$ ) of the given CP HS. A one-parameter group of shifts turns a CP HS into a homogeneous CP HS, as defined below.

1.6 Definition. (a) A homogeneous continuous product of Hilbert spaces ('HCP HS', for short) is $\left(\left(H_{s, t}\right)_{-\infty \leq s<t \leq \infty},\left(W_{r, s, t}\right)_{-\infty \leq r<s<t \leq \infty}\right.$, $\left.\left(\theta_{s, t}^{u}\right)_{u \in \mathbb{R},-\infty \leq s<t \leq \infty}\right)$ where $\left(\left(H_{s, t}\right)_{s<t},\left(W_{r, s, t}\right)_{r<s<t}\right)$ is a CP HS, and $\left(\theta_{s, t}^{u}\right)_{u ; s<t}$ is a family of unitary operators

$$
\theta_{s, t}^{u}: H_{s, t} \rightarrow H_{s+u, t+u}
$$

such that $\theta_{s, t}^{0}=\mathbf{1}_{s, t}$, and

(a1) the diagram

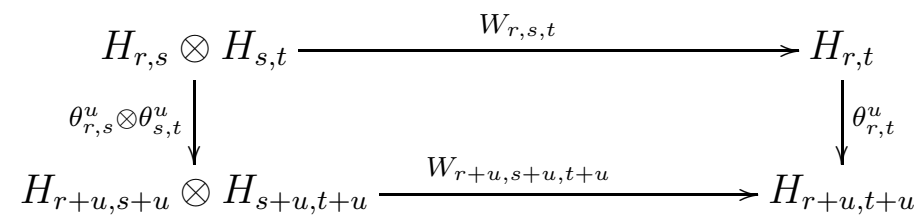

is commutative whenever $u \in \mathbb{R},-\infty \leq r<s<t \leq \infty$;

(a2) the diagram

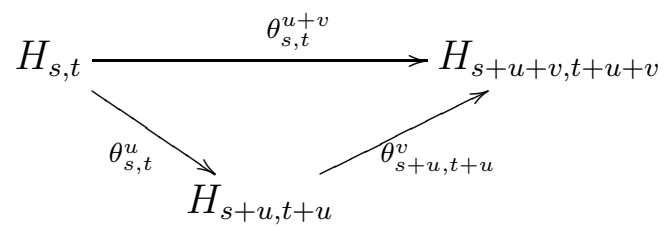


is commutative whenever $u, v \in \mathbb{R},-\infty \leq s<t \leq \infty$;

(a3) operators $\theta_{-\infty, \infty}^{u}$ are a strongly continuous unitary group; that is, $\theta_{-\infty, \infty}^{u}=e^{i u X}$ for some self-adjoint operator $X$ on $H_{-\infty, \infty}$.

The HCP HS is called tail trivial, if the underlying CP HS is tail trivial.

(b) An isomorphism of one HCP HS $\left(\left(H_{s, t}\right)_{s<t},\left(W_{r, s, t}\right)_{r<s<t},\left(\theta_{s, t}^{u}\right)_{u ; s<t}\right)$ to another HCP HS $\left(\left(H_{s, t}^{\prime}\right)_{s<t},\left(W_{r, s, t}^{\prime}\right)_{r<s<t},\left(\theta_{s, t}^{\prime u}\right)_{u ; s<t}\right)$ is an isomorphism $\left(U_{s, t}\right)_{s<t}$ of the CP HS $\left(\left(H_{s, t}\right)_{s<t},\left(W_{r, s, t}\right)_{r<s<t}\right)$ to the CP HS $\left(\left(H_{s, t}^{\prime}\right)_{s<t},\left(W_{r, s, t}^{\prime}\right)_{r<s<t}\right)$ (as defined by 1.5(b)) such that the diagram

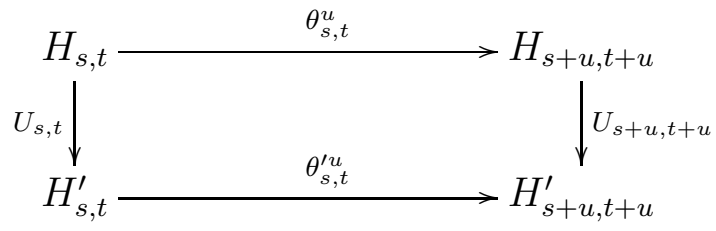

is commutative whenever $u \in \mathbb{R},-\infty \leq s<t \leq \infty$.

Given an HCP HS, we may take $H_{-}=H_{-\infty, 0}, H_{+}=H_{0, \infty}$, and consider the unitary group $\left(W_{-\infty, 0, \infty}^{-1} e^{i u X} W_{-\infty, 0, \infty}\right)_{u \in \mathbb{R}}$ on $H_{-} \otimes H_{+}$, then (1.1) and (1.2) are satisfied; indeed, (1.2) follows from the lemma below.

\begin{tabular}{|c|c|}
\hline & $\alpha_{u}^{+}(B)$ \\
\hline$\widehat{W_{0, u, \infty} \uparrow}$ & $\sqrt{W_{0, u, \infty}^{-1}}$ \\
\hline $\mathbf{1}_{0, u}$ & $\theta_{0, \infty}^{u} B \theta_{u, \infty}^{-u}$ \\
\hline 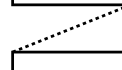 & $\theta_{u, \infty}^{-u}>\bar{\theta}_{0, \infty}^{u}$ \\
\hline
\end{tabular}

1.7 Lemma. For every HCP HS $\left(\left(H_{s, t}\right)_{s<t},\left(W_{r, s, t}\right)_{r<s<t},\left(\theta_{s, t}^{u}\right)_{u ; s<t}\right)$, every $u \geq 0, A \in \mathcal{B}\left(H_{-\infty, 0}\right), B \in \mathcal{B}\left(H_{0, \infty}\right)$,

$$
\alpha_{-u}(A \otimes \mathbf{1})=\alpha_{u}^{-}(A) \otimes \mathbf{1}, \quad \alpha_{u}(\mathbf{1} \otimes B)=\mathbf{1} \otimes \alpha_{u}^{+}(B),
$$

where

$$
\begin{gathered}
\alpha_{u}^{-}(A)=W_{-\infty, u, 0}\left(\theta_{-\infty, 0}^{u} A \theta_{-\infty, u}^{-u} \otimes \mathbf{1}_{u, 0}\right) W_{-\infty, u, 0}^{-1}, \\
\alpha_{u}^{+}(B)=W_{0, u, \infty}\left(\mathbf{1}_{0, u} \otimes \theta_{0, \infty}^{u} B \theta_{u, \infty}^{-u}\right) W_{0, u, \infty}^{-1} .
\end{gathered}
$$

Proof. Only the formula for $\alpha^{+}$will be proven; the formula for $\alpha^{-}$is similar. We have

$$
\begin{gathered}
\alpha_{u}(\mathbf{1} \otimes B)=W_{-\infty, 0, \infty}^{-1} e^{i u X} W_{-\infty, 0, \infty}(\mathbf{1} \otimes B) W_{-\infty, 0, \infty}^{-1} e^{-i u X} W_{-\infty, 0, \infty}, \\
e^{i u X} W_{-\infty, 0, \infty}=\theta_{-\infty, \infty}^{u} W_{-\infty, 0, \infty}=W_{-\infty, 0, \infty}\left(\theta_{-\infty, 0}^{u} \otimes \theta_{0, \infty}^{u}\right)
\end{gathered}
$$


by $1.6(\mathrm{c}, \mathrm{a})$;

$$
W_{-\infty, 0, \infty}\left(\mathbf{1}_{-\infty, 0} \otimes W_{0, u, \infty}\right)=W_{-\infty, u, \infty}\left(W_{-\infty, 0, u} \otimes \mathbf{1}_{u, \infty}\right)
$$

by $1.5(a 1) ;$ thus,

$$
\begin{aligned}
& W_{-\infty, 0, \infty}^{-1} e^{i u X} W_{-\infty, 0, \infty}=W_{-\infty, 0, \infty}^{-1} W_{-\infty, u, \infty}\left(\theta_{-\infty, 0}^{u} \otimes \theta_{0, \infty}^{u}\right)= \\
& =\left(\mathbf{1}_{-\infty, 0} \otimes W_{0, u, \infty}\right)\left(W_{-\infty, 0, u} \otimes \mathbf{1}_{u, \infty}\right)^{-1}\left(\theta_{-\infty, 0}^{u} \otimes \theta_{0, \infty}^{u}\right) .
\end{aligned}
$$

On the other hand,

$$
\left(\theta_{-\infty, 0}^{u} \otimes \theta_{0, \infty}^{u}\right)\left(\mathbf{1}_{-\infty, 0} \otimes B\right)\left(\theta_{-\infty, 0}^{u} \otimes \theta_{0, \infty}^{u}\right)^{-1}=\mathbf{1}_{-\infty, u} \otimes\left(\theta_{0, \infty}^{u} B\left(\theta_{0, \infty}^{u}\right)^{-1}\right) ;
$$

thus

$$
\begin{gathered}
\left(W_{-\infty, 0, u} \otimes \mathbf{1}_{u, \infty}\right)^{-1}\left(\theta_{-\infty, 0}^{u} \otimes \theta_{0, \infty}^{u}\right)\left(\mathbf{1}_{-\infty, 0} \otimes B\right)\left(\theta_{-\infty, 0}^{u} \otimes \theta_{0, \infty}^{u}\right)^{-1}\left(W_{-\infty, 0, u} \otimes \mathbf{1}_{u, \infty}\right) \\
=\left(W_{-\infty, 0, u} \otimes \mathbf{1}_{u, \infty}\right)^{-1}\left(\mathbf{1}_{-\infty, u} \otimes \theta_{0, \infty}^{u} B\left(\theta_{0, \infty}^{u}\right)^{-1}\right)\left(W_{-\infty, 0, u} \otimes \mathbf{1}_{u, \infty}\right)= \\
=\mathbf{1}_{-\infty, 0} \otimes \mathbf{1}_{0, u} \otimes \theta_{0, \infty}^{u} B\left(\theta_{0, \infty}^{u}\right)^{-1}
\end{gathered}
$$

and so,

$$
\begin{gathered}
\alpha_{u}(\mathbf{1} \otimes B)=\left(\mathbf{1}_{-\infty, 0} \otimes W_{0, u, \infty}\right)\left(\mathbf{1}_{-\infty, 0} \otimes \mathbf{1}_{0, u} \otimes \theta_{0, \infty}^{u} B\left(\theta_{0, \infty}^{u}\right)^{-1}\right)\left(\mathbf{1}_{-\infty, 0} \otimes W_{0, u, \infty}\right)^{-1} \\
=\mathbf{1}_{-\infty, 0} \otimes W_{0, u, \infty}\left(\mathbf{1}_{0, u} \otimes \theta_{0, \infty}^{u} B\left(\theta_{0, \infty}^{u}\right)^{-1}\right) W_{0, u, \infty}^{-1}=\mathbf{1}_{-\infty, 0} \otimes \alpha_{u}^{+}(B) .
\end{gathered}
$$

1.8 Corollary. Let $\left(\left(H_{s, t}\right)_{s<t},\left(W_{r, s, t}\right)_{r<s<t},\left(\theta_{s, t}^{u}\right)_{u ; s<t}\right)$ be a tail trivial HCP HS. Then the unitary group $\left(W_{-\infty, 0, \infty}^{-1} \theta_{-\infty, \infty}^{t} W_{-\infty, 0, \infty}\right)_{t \in \mathbb{R}}$ on $H_{-\infty, 0} \otimes H_{0, \infty}$ and the algebra $\mathcal{B}\left(H_{-\infty, 0}\right) \otimes \mathbf{1}$ form a history (as defined in [A, Sect. 1.2]).

Proof. Property (1.2) follows from Lemma 1.7; tail triviality follows from Definition 1.5(a2).

Every HCP HS (tail trivial or not) leads (via its $E_{0}$-semigroup $\alpha^{+}$) to a product system $(\mathcal{E}(t))_{t>0}$. As was said, an element of $\mathcal{E}(t)$ is an operator $T \in \mathcal{B}\left(H_{+}\right)$such that $\alpha_{t}(A) T=T A$ for all $A \in \mathcal{B}\left(H_{+}\right)$. According to Lemma 1.7 we rewrite it as

$$
W_{0, t, \infty} \underbrace{\left(\mathbf{1}_{0, t} \otimes \theta_{0, \infty}^{t}\right)\left(\mathbf{1}_{0, t} \otimes A\right)\left(\mathbf{1}_{0, t} \otimes \theta_{0, \infty}^{t}\right)^{-1}}_{\left(\mathbf{1}_{0, t} \otimes \theta_{0, \infty}^{t} A \theta_{t, \infty}^{-t}\right)} W_{0, t, \infty}^{-1} T=T A,
$$

or just

$$
\left(\mathbf{1}_{0, t} \otimes A\right) \tilde{T}=\tilde{T} A
$$


where

$$
\tilde{T}=\left(\mathbf{1}_{0, t} \otimes \theta_{t, \infty}^{-t}\right) W_{0, t, \infty}^{-1} T, \quad \tilde{T}: H_{0, \infty} \rightarrow H_{0, t} \otimes H_{0, \infty} .
$$

Applying it to a one-dimensional operator $A$ we see that $\tilde{T} x \in H_{0, t} \otimes x$ for each $x \in H_{0, \infty}$; therefore $\tilde{T}$ must be of the form

$$
\tilde{T} x=y \otimes x
$$

for some $y \in H_{0, t}$. Also, for every $y$, such $\tilde{T}$ belongs to $\mathcal{E}(t)$. We have a natural unitary map from $H_{0, t}$ to $\mathcal{E}(t)$ (the latter consists of operators, but is isometric to a Hilbert space); namely, $y \mapsto T y$,

$$
T_{y} x=W_{0, t, \infty}\left(\mathbf{1}_{0, t} \otimes \theta_{0, \infty}^{t}\right)(y \otimes x)=W_{0, t, \infty}\left(y \otimes \theta_{0, \infty}^{t} x\right) .
$$

Let $\tilde{T}_{1} \in \mathcal{E}\left(t_{1}\right), \tilde{T}_{2} \in \mathcal{E}\left(t_{2}\right), \tilde{T}_{1} x=y_{1} \otimes x, \tilde{T}_{2} x=y_{2} \otimes x, y_{1} \in H_{0, t_{1}}$, $y_{2} \in H_{0, t_{2}}$, and $T=T_{2} T_{1}$. Then

$$
\begin{gathered}
T x=T_{2} T_{1} x=W_{0, t_{2}, \infty}\left(y_{2} \otimes \theta_{0, \infty}^{t_{2}} T_{1} x\right)= \\
\left.=W_{0, t_{2}, \infty}(y_{2} \otimes \underbrace{W_{t_{2}, t_{1}+t_{2}, \infty}\left(\theta_{0, t_{1}}^{t_{2}} \otimes \theta_{t_{1}, \infty}^{t_{2}}\right.}_{\theta_{0, \infty}^{t_{2}} W_{0, t_{1}, \infty}})\left(y_{1} \otimes \theta_{0, \infty}^{t_{1}} x\right)\right)= \\
=W_{0, t_{2}, \infty}\left(y_{2} \otimes W_{t_{2}, t_{1}+t_{2}, \infty}\left(\theta_{0, t_{1}}^{t_{2}} y_{1} \otimes \theta_{0, \infty}^{t_{1}+t_{2}} x\right)\right)= \\
=W_{0, t_{1}+t_{2}, \infty}\left(W_{0, t_{2}, t_{1}+t_{2}}\left(y_{2} \otimes \theta_{0, t_{1}}^{t_{2}} y_{1}\right) \otimes \theta_{0, \infty}^{t_{1}+t_{2}} x\right),
\end{gathered}
$$

that is,

$$
\tilde{T} x=y \otimes x, \quad \text { where } y=W_{0, t_{2}, t_{1}+t_{2}}\left(y_{2} \otimes \theta_{0, t_{1}}^{t_{2}} y_{1}\right) .
$$

The latter is the multiplication of the product system, transplanted from $(\mathcal{E}(t))_{t>0}$ to $\left(H_{0, t}\right)_{t>0}$. We may transplant also the Borel structure, which leads to the following conclusion.

1.9 Theorem. Let $\left(\left(H_{s, t}\right)_{s<t},\left(W_{r, s, t}\right)_{r<s<t},\left(\theta_{s, t}^{u}\right)_{u ; s<t}\right)$ be a HCP HS. Then the disjoint union

$$
E=\biguplus_{t>0} H_{0, t}=\left\{(t, x): 0<t<\infty, x \in H_{0, t}\right\}
$$

is a product system (as defined in [A, Def. 3.2.1]), being equipped with the binary operation

$$
((s, x),(t, y)) \mapsto W_{0, s, s+t}\left(x \otimes \theta_{0, t}^{s} y\right)
$$

and the Borel structure induced by such an embedding into operators on $H_{0, \infty}$ :

$$
(s, x) \mapsto\left(y \mapsto W_{0, s, \infty}\left(x \otimes \theta_{0, \infty}^{s} y\right)\right)
$$


Our definition of CP HS stipulates Hilbert spaces $H_{s, t}$ not only for bounded intervals $(s, t)$, but also for unbounded intervals $(-\infty, t),(s, \infty)$ and $(-\infty, \infty)$. These unbounded intervals are more important than it would seem! We may define a local $C P H S$ by excluding unbounded intervals from Definition 1.5. Even under tail triviality (item (a2) of Def. 1.5), a global CP HS is far from being uniquely determined by its local CP HS. Indeed, asymptotic behavior at infinity is evidently missing in a local CP HS. Strangely enough, something else, seemingly local, is also missing. A global CP HS induces a natural Borel structure on its product system $\uplus H_{0, t}$ (see Theorem 1.9), but a local CP HS does not, for the following reason.

Let $\varphi: \mathbb{R} \rightarrow \mathbb{R}$ be a function, additive in the sense that $\varphi(s+t)=\varphi(s)+$ $\varphi(t)$ for all $s, t$. It is well-known that such $\varphi$ is either linear, $\varphi(t)=c t$, or quite pathologic (non-measurable); the latter case is possible (which follows easily from existence of a Hamel basis). Let $\left(\left(H_{s, t}\right)_{s<t},\left(W_{r, s, t}\right)_{r<s<t},\left(\theta_{s, t}^{u}\right)_{u ; s<t}\right)$ be a local HCP HS.'f Define $U_{s, t} \in \mathcal{B}\left(H_{s, t}\right)$ as the scalar operator $U_{s, t}=$ $e^{i \varphi(t-s)} \mathbf{1}_{s, t}$; then $\left(U_{s, t}\right)_{s<t}$ is an automorphism of the local HCP HS (as defined by Def. 1.6(b), but excluding unbounded intervals). If the local HCP HS is induced by a global HCP HS, then $\uplus H_{0, t}$ has its natural Borel structure, but the transformation of $\uplus H_{0, t}$ that multiplies each $H_{0, t}$ by $e^{i \varphi(t)}$ is not Borel, unless $\varphi$ is Borel (therefore, linear). Such a pathologic automorphism of the local HCP HS cannot be extended to an automorphism of the global HCP HS. Here is a simple explanation: a family of scalar operators $U_{s, t}=u_{s, t} \mathbf{1}, u_{s, t} \in \mathbb{C},\left|u_{s, t}\right|=1$, is an automorphism if and only if $u_{r, s} u_{s, t}=u_{r, t}$ and $u_{r, s}=u_{r+t, s+t}$. Thus, $u_{r, s}=\overline{f(r)} f(s)$ for some $f$ such that $\overline{f(r)} f(s)=\overline{f(r+t)} f(s+t)$. If the case $s=\infty$ is admitted, we get $\overline{f(r)} f(\infty)=\overline{f(r+t)} f(\infty)$, and $f$ must be constant!

Or rather, $f$ must be constant on $(-\infty, \infty)$, not $[-\infty, \infty]$. We may take two numbers $u_{-}, u_{+} \in \mathbb{C},\left|u_{-}\right|=1,\left|u_{+}\right|=1$, and define $u_{-\infty, t}=u_{-}$, $u_{t, \infty}=u_{+}$for $t \in \mathbb{R}$; of course, $u_{-\infty, \infty}=u_{-} u_{+}$and $u_{r, s}=1$ for $r, s \in \mathbb{R}$. Then $\left(u_{s, t}\right)_{s<t}$ is an automorphism of the global HCP HS, whose restriction to the local HCP HS is trivial. We see that restriction (of automorphisms, from global to local HCP HS) is neither surjective nor injective.

1.10 Lemma. Let $\left(\left(H_{s, t}\right)_{-\infty \leq s<t \leq \infty},\left(W_{r, s, t}\right)_{-\infty \leq r<s<t \leq \infty},\left(\theta_{s, t}^{u}\right)_{u \in \mathbb{R},-\infty \leq s<t \leq \infty}\right)$ and $\quad\left(\left(H_{s, t}^{\prime}\right)_{-\infty \leq s<t \leq \infty},\left(W_{r, s, t}^{\prime}\right)_{-\infty \leq r<s<t \leq \infty},\left(\theta_{s, t}^{\prime u}\right)_{u \in \mathbb{R},-\infty \leq s<t \leq \infty}\right) \quad$ be two HCPs HS. If the corresponding product systems $\uplus H_{0, t}$, $\uplus H_{0, t}^{\prime}$ are isomorphic then the corresponding local HCPs HS $\quad\left(\left(H_{s, t}\right)_{-\infty<s<t<\infty},\left(W_{r, s, t}\right)_{-\infty<r<s<t<\infty},\left(\theta_{s, t}^{u}\right)_{u \in \mathbb{R},-\infty<s<t<\infty}\right) \quad$ and $\left(\left(H_{s, t}^{\prime}\right)_{-\infty<s<t<\infty},\left(W_{r, s, t}^{\prime}\right)_{-\infty<r<s<t<\infty},\left(\theta_{s, t}^{\prime u}\right)_{u \in \mathbb{R},-\infty<s<t<\infty}\right)$ are isomorphic.

\footnotetext{
${ }^{4}$ It is not clear, how to 'localize' item (a3) of Def. 1.6. Anyway, we may restrict ourselves to local HCPs HS that can be extended to global HCPs HS.
} 
Proof. Isomorphism between $\uplus H_{0, t}$ and $\uplus H_{0, t}^{\prime}$ is a family $\left(U_{0, t}\right)_{0<t<\infty}$ of unitary operators $U_{0, t}: H_{0, t} \rightarrow H_{0, t}^{\prime}$ such that the diagram

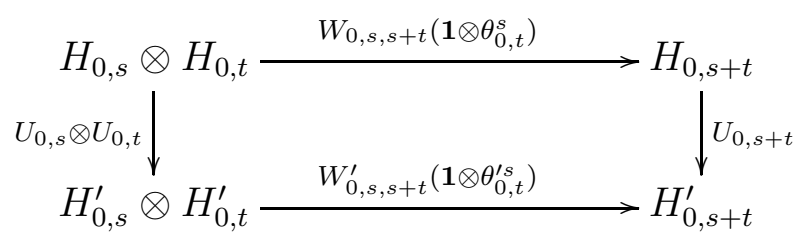

is commutative for all $s, t \in(0, \infty)$. In addition, $\left(U_{0, t}\right)_{0<t<\infty}$ satisfies a Borel measurability condition, but this one will not be used. We define $U_{s, t}: H_{s, t} \rightarrow$ $H_{s, t}^{\prime}$ for $-\infty<s<t<\infty$ by

$$
U_{s, t}=\theta_{0, t-s}^{s} U_{0, t-s} \theta_{s, t}^{-s}
$$

We have

$$
\begin{gathered}
U_{r, t} W_{r, s, t}=\theta_{0, t-r}^{\prime r} U_{0, t-r} \theta_{r, t}^{-r} W_{r, s, t}= \\
=\theta_{0, t-r}^{\prime r} U_{0, t-r} W_{0, s-r, t-r}\left(\theta_{r, s}^{-r} \otimes \theta_{s, t}^{-r}\right)= \\
=\theta_{0, t-r}^{\prime r} U_{0, t-r} W_{0, s-r, t-r}\left(\mathbf{1} \otimes \theta_{0, t-s}^{s-r}\right)\left(\theta_{r, s}^{-r} \otimes \theta_{s, t}^{-s}\right)= \\
=\theta_{0, t-r}^{\prime r} W_{0, s-r, t-r}^{\prime}\left(\mathbf{1} \otimes \theta_{0, t-s}^{s-r}\right)\left(U_{0, s-r} \otimes U_{0, t-s}\right)\left(\theta_{r, s}^{-r} \otimes \theta_{s, t}^{-s}\right)= \\
=W_{r, s, t}^{\prime}\left(\theta_{0, s-r}^{\prime r} \otimes \theta_{s-r, t-r}^{\prime r}\right)\left(\mathbf{1} \otimes \theta_{0, t-s}^{s-r}\right)\left(U_{0, s-r} \otimes U_{0, t-s}\right)\left(\theta_{r, s}^{-r} \otimes \theta_{s, t}^{-s}\right)= \\
=W_{r, s, t}^{\prime}\left(\theta_{0, s-r}^{\prime r} U_{0, s-r} \theta_{r, s}^{-r} \otimes \theta_{s-r, t-r}^{\prime r} \theta_{0, t-s}^{s-r} U_{0, t-s} \theta_{s, t}^{-s}\right)= \\
=W_{r, s, t}^{\prime}\left(U_{r, s} \otimes U_{s, t}\right),
\end{gathered}
$$

which means commutativity of the diagram of Def. 1.5(b). Also,

$$
\begin{aligned}
U_{s+u, t+u} \theta_{s, t}^{u}=\theta_{0, t-s}^{\prime s+u} U_{0, t-s} & \theta_{s+u, t+u}^{-s-u} \theta_{s, t}^{u}= \\
& =\theta_{0, t-s}^{\prime s+u} U_{0, t-s} \theta_{s, t}^{-s}=\theta_{s, t}^{\prime u} \theta_{0, t-s}^{\prime s} U_{0, t-s} \theta_{s, t}^{-s}=\theta_{s, t}^{\prime u} U_{s, t},
\end{aligned}
$$

which means commutativity of the diagram of Def. 1.6(b).

In principle, it would be more natural to define a CP HS as local (rather than global) and equipped with a Borel structure (rather than the purely algebraic structure) in the spirit of [A, Def. 3.2.1]. However, given the aim to construct non-isomorphic product systems, we consider

global HCPs HS up to local isomorphisms;

that is, our objects are global HCPs HS, but our isomorphisms are isomorphisms of corresponding local CPs HS (no Borel structure stipulated). It means that 
(a) we deal with a subclass of the class of all product systems,

(b) on that subclass we introduce an equivalence relation coarser than isomorphism of product systems.

Therefore, existence of many nonequivalent objects within our framework implies existence of (at least) equally many non-isomorphic product systems.

It was noted (after Theorem 1.9) that the Borel structure on a local CP HS emerges via globality and homogeneity. It is natural to ask what happens to the Borel structure in a local inhomogeneous setup. The next result presents an answer which will not be used, and I omit the proof; the reader may skip to Sect. 2.

1.11 Theorem. For every local CP HS $\left(\left(H_{s, t}\right)_{-\infty<s<t<\infty},\left(W_{r, s, t}\right)_{-\infty<r<s<t<\infty}\right)$ there exists a Borel structure on the disjoint union $\uplus H_{s, t}$ of all these Hilbert spaces such that

(a) $\uplus H_{s, t}$ is a standard Borel space;

(b) the projection $\uplus H_{s, t} \rightarrow\{(s, t):-\infty<s<t<\infty\}$ (sending each $H_{s, t}$ to $(s, t))$ is a Borel map;

(c) the map $(x, y) \mapsto U_{r, s, t}(x \otimes y)$ from the Borel subset $\cup_{r, s, t} H_{r, s} \times H_{s, t}$ of $\left(\uplus H_{s, t}\right) \times\left(\uplus H_{s, t}\right)$ to $\uplus H_{s, t}$ is a Borel map;

$(\mathrm{d})$ there exists a sequence $\left(e_{n}\right)$ of Borel maps $e_{n}:\{(s, t): 0 \leq s<t<$ $\infty\} \rightarrow \uplus H_{s, t}$ such that the sequence $\left(e_{1}(s, t), e_{2}(s, t), \ldots\right)$ is an orthonormal basis of $H_{s, t}$ for each $(s, t)$ satisfying $\operatorname{dim} H_{s, t}=\infty$. Otherwise, the first $n=\operatorname{dim} H_{s, t}$ elements of the sequence are such a basis, and other elements vanish.

Do not think that the Borel structure is unique. Rotating each $H_{s, t}$ by a phase factor $u_{s, t}=\overline{f(s)} f(t), f: \mathbb{R} \rightarrow\{z \in \mathbb{C}:|z|=1\}$, we get an automorphism of the local CP HS, even if $f$ is not a Borel function.

\section{Some invariants}

The fundamental problem in the theory of $E_{0^{-}}$ semigroups is to find a complete set of computable invariants for cocycle conjugacy. There is some optimism that such a classification is possible, but we are far from achieving that goal.

W. Arveson [A, Sect. 3]

In the absence of a complete set of computable invariants, some useful specific invariants are needed, if we want to present non-isomorphic product systems (or $E_{0}$-semigroups). However, I consider continuous products 
of Hilbert spaces (CPs HS) instead of product systems. Moreover, I consider CPs HS over $[0,1]$ (rather than $(-\infty, \infty)$ ) and waive homogeneity. Ultimately, my objects are 'global HCPs HS up to local isomorphisms', as explained in Sect. 1. But for now it does not harm to define invariants on a wider class of objects. A local isomorphism of global HCPs HS evidently induces an isomorphism of corresponding CPs HS over [0,1]. Such CPs HS (and their isomorphisms) are defined similarly to Def. 1.5; the only modification is that $r, s, t \in[0,1]$ rather than $[-\infty, \infty]$.

So, an arbitrary CP HS $\left(H_{s, t}\right)_{0 \leq s<t \leq 1}$ is considered. Instead of $W_{r, s, t}$ : $H_{r, s} \otimes H_{s, t} \rightarrow H_{r, t}$ I'll write simply $H_{r, s} \otimes H_{s, t}=H_{r, t}$, glueing these spaces together and treating $W_{r, s, t}$ as identity maps. (The reader could restore all needed $W_{r, s, t}$, thus returning to the rigorous but cumbersome style of Sect. 1. Associativity stipulated by Def. 1.5(a1) eliminates ambiguity.)

A subset of $[0,1]$ will be called elementary, if it is the union of a finite number of intervals. We treat elementary sets modulo finite sets; say, $(0.3,0.4) \cup(0.6,0.7) \cup(0.7,0.8)$ and $[0.3,0.4) \cup\{0.5\} \cup[0.6,0.8]$ are equivalent elementary sets. The general form of an (equivalence class of) elementary set is

$$
E=\left(s_{1}, t_{1}\right) \cup \cdots \cup\left(s_{n}, t_{n}\right), \quad 0 \leq s_{1}<t_{1}<\cdots<s_{n}<t_{n} \leq 1
$$

$n=0,1,2, \ldots(n=0$ means $E=\emptyset)$. These equivalence classes are a Boolean algebra $\mathcal{E}$.

We define for each $E \in \mathcal{E}$ a Hilbert space

$$
H_{E}=H_{s_{1}, t_{1}} \otimes \ldots \otimes H_{s_{n}, t_{n}}
$$

and observe that

$$
H_{E \cup F}=H_{E} \otimes H_{F} \quad \text { whenever } E, F \in \mathcal{E}, E \cap F=\emptyset \text {. }
$$

In particular,

$$
H_{0,1}=H_{[0,1]}=H_{E} \otimes H_{[0,1] \backslash E} ;
$$

accordingly, we define

$$
\mathcal{A}_{E}=\mathcal{B}\left(H_{E}\right) \otimes \mathbf{1}_{[0,1] \backslash E} \subset \mathcal{A}_{0,1}=\mathcal{B}\left(H_{0,1}\right)
$$

(here $\mathbf{1}_{[0,1] \backslash E}$ is the identity operator on $H_{[0,1] \backslash E}$ ), getting

$$
\begin{gathered}
\mathcal{A}_{E} \otimes \mathcal{A}_{F}=\mathcal{A}_{E \cup F} \quad \text { for } E \cap F=\emptyset, \\
\mathcal{A}_{E}^{\prime}=\mathcal{A}_{[0,1] \backslash E} .
\end{gathered}
$$


2.2 Definition. A sequence $\left(E_{k}\right)$ of (equivalence classes of) elementary sets $E_{1}, E_{2}, \cdots \in \mathcal{E}$ is infinitesimal for the CP HS $\left(H_{s, t}\right)_{0 \leq s<t \leq 1}$, if

$$
\sup _{A \in \mathcal{A}_{k},\|A\| \leq 1}|\operatorname{tr}(A R)| \rightarrow 0 \quad \text { for } k \rightarrow \infty
$$

for each trace-class operator $R \in \mathcal{B}\left(H_{0,1}\right)$ satisfying $\operatorname{tr}(R)=0$; here $\mathcal{A}_{k}=$ $\mathcal{A}_{E_{k}} \subset \mathcal{A}_{0,1}$.

Clearly, such a definition can be used in a much more general situation, for an arbitrary sequence of von Neumann subalgebras of $\mathcal{B}(H)$. A decreasing sequence $\left(E_{1} \supset E_{2} \supset \ldots\right.$, thus $\left.\mathcal{A}_{1} \supset \mathcal{A}_{2} \supset \ldots\right)$ is infinitesimal if and only if the intersection of all $\mathcal{A}_{k}$ is trivial (scalar operators only); this fact will not be used, and I omit the proof. Rather, a non-monotone sequence of such a form will be used:

$$
E_{n}=\bigcup_{k=0}^{n-1}\left(\frac{1}{n}\left(k+\frac{1-\varepsilon_{n}}{2}\right), \frac{1}{n}\left(k+\frac{1+\varepsilon_{n}}{2}\right)\right),
$$

the union of $n$ equidistant intervals, of total length mes $E_{n}=\varepsilon_{n}$. The class of sequences $\left(\varepsilon_{n}\right)$ such that $\left(E_{n}\right)$ is infinitesimal, is an invariant of a CP HS. Continuum of type III product systems will be presented by means of that invariant.

Let $\left(H_{s, t}\right)_{s<t}$ be a CP HS over [0,1], and subspaces $H_{s, t}^{\prime} \subset H_{s, t}, \operatorname{dim} H_{s, t}^{\prime}>$ 0 , satisfy $H_{r, s}^{\prime} \otimes H_{s, t}^{\prime}=H_{r, t}^{\prime}$ whenever $0 \leq r<s<t \leq 1$; then $\left(H_{s, t}^{\prime}\right)_{s<t}$ is another CP HS on $[0,1]$, embedded into $\left(H_{s, t}\right)_{s<t}$. An invariant for such a pair $\left(\left(H_{s, t}\right)_{s<t},\left(H_{s, t}^{\prime}\right)_{s<t}\right)$ is proposed below. Of course, it is an invariant of a pair (embedding), not of a single CP HS. However, in some situations (especially relevant to type $I I$ product systems) it leads to some invariants of a single CP HS. It happens, when subspaces $H_{s, t}^{\prime}$ can be unambiguously defined in terms of the given CP HS $\left(H_{s, t}\right)_{s<t}$. Especially, we may ask about one-dimensional $H_{s, t}^{\prime}$.

2.3 Definition. Let $\left(H_{s, t}\right)_{s<t}$ be a CP HS over $[0,1]$.

(a) A decomposable vector (of the CP HS) is a vector $\psi \in H_{0,1}, \psi \neq 0$, such that for every $t \in(0,1), \psi$ is of the form $\psi^{\prime} \otimes \psi^{\prime \prime}$ for some $\psi^{\prime} \in H_{0, t}$, $\psi^{\prime \prime} \in H_{t, 1}$.

(b) The CP HS is of type $I$, if decomposable vectors span the whole $H_{0,1}$ (as a closed linear space).

(c) The CP HS is of type III, if it has no decomposable vectors.

(d) The CP HS is of type II, if it does not belong to types I, III.

(e) The CP HS is of type $I I_{0}$, if it has a decomposable vector, and all decomposable vectors belong to a single one-dimensional subspace of $H_{0,1}$. 
For a CP HS of type $I I_{0}$ (examples will be given in Sect. 6), one-dimensional subspaces $H_{s, t}^{\prime} \subset H_{s, t}$ satisfying $H_{r, s}^{\prime} \otimes H_{s, t}^{\prime}=H_{r, t}^{\prime}$ are unique; in such a case, an invariant of a pair leads to an invariant of a CP HS itself. The invariant is described below for an arbitrary pair, but the reader may restrict himself to the case of $\operatorname{dim} H_{s, t}^{\prime}=1$ and type $I I_{0}$.

Given an elementary set (2.1), we introduce the subspace $\left(H / H^{\prime}\right)_{E}=H_{E} \otimes H_{[0,1] \backslash E}^{\prime}=H_{0, s_{1}}^{\prime} \otimes H_{s_{1}, t_{1}} \otimes H_{t_{1}, s_{2}}^{\prime} \otimes \ldots \otimes H_{s_{n}, t_{n}} \otimes H_{t_{n}, 1}^{\prime} \subset H_{0,1}$, and the corresponding projection

$$
Q_{E}=\mathbf{1}_{E} \otimes P_{[0,1] \backslash E}^{\prime}=P_{0, s_{1}}^{\prime} \otimes \mathbf{1}_{s_{1}, t_{1}} \otimes P_{t_{1}, s_{2}}^{\prime} \otimes \ldots \otimes \mathbf{1}_{s_{n}, t_{n}} \otimes P_{t_{n}, 1}^{\prime} \in \mathcal{A}_{0,1}
$$

where $P_{s, t}^{\prime} \in \mathcal{A}_{s, t}$ is the projection onto $H_{s, t}^{\prime}$. Clearly,

$$
\begin{gathered}
\left(H / H^{\prime}\right)_{E_{1} \cap E_{2}}=\left(H / H^{\prime}\right)_{E_{1}} \cap\left(H / H^{\prime}\right)_{E_{2}}, \\
Q_{E_{1} \cap E_{2}}=Q_{E_{1}} Q_{E_{2}}=Q_{E_{2}} Q_{E_{1}}
\end{gathered}
$$

for all $E_{1}, E_{2} \in \mathcal{E}$. (Note however that in general $Q_{E_{1} \cup E_{2}}$ is strictly larger than $Q_{E_{1}}+Q_{E_{2}}$ for $E_{1} \cap E_{2}=\emptyset$.) Thus, $\left\{Q_{E}: E \in \mathcal{E}\right\}$ is a commuting set of projections, and $H_{0,1}$ decomposes into the corresponding direct integral. We'll describe the relevant measure space explicitly, using the following quite general result of measure theory.

2.4 Lemma. Let $X$ be a compact topological space, $\mathcal{A}$ an algebraf of subsets of $X$, and $\mu: \mathcal{A} \rightarrow[0, \infty)$ an additive function satisfying the condition

(a) [regularity] for every $A \in \mathcal{A}$ and $\varepsilon>0$ there exists $B \in \mathcal{A}$ such that $\bar{B} \subset A$ (here $\bar{B}$ is the closure of $B$ ) and $\mu(B) \geq \mu(A)-\varepsilon$.

Then $\mu$ has a unique extension to a measure on the $\sigma$-field generated by $\mathcal{A}$.

Proof. Due to a well-known theorem, it is enough to prove that $\mu$ is $\sigma$-additive on $\mathcal{A}$. Let $A_{1} \supset A_{2} \supset \ldots, A_{1}, A_{2}, \cdots \in \mathcal{A}, \cap A_{k}=\emptyset$; we have to prove that $\mu\left(A_{k}\right) \rightarrow 0$. Given $\varepsilon>0$, we can choose $B_{k} \in \mathcal{A}$ such that $\bar{B}_{k} \subset A_{k}$ and $\mu\left(B_{k}\right) \geq \mu\left(A_{k}\right)-2^{-k} \varepsilon$. Due to compactness, the relation $\cap \bar{B}_{k} \subset \cap A_{k}=\emptyset$ implies $\bar{B}_{1} \cap \cdots \cap \bar{B}_{n}=\emptyset$ for some $n$. Thus, $\mu\left(A_{n}\right)=\mu\left(A_{1} \cap \cdots \cap A_{n}\right) \leq$ $\mu\left(B_{1} \cap \cdots \cap B_{n}\right)+\mu\left(A_{1} \backslash B_{1}\right)+\cdots+\mu\left(A_{n} \backslash B_{n}\right)<\varepsilon$.

2.5 Remark. All $A \in \mathcal{A}$ such that $A$ and $X \backslash A$ both satisfy the regularity condition, are a subalgebra of $\mathcal{A}$. (The proof is left to the reader.) Therefore it is enough to check the condition for $A$ and $X \backslash A$ where $A$ runs over a set that generates the algebra $\mathcal{A}$.

\footnotetext{
${ }^{5}$ That is, $\mathcal{A}$ is closed under complement and finite union.
} 
2.6 Lemma. Let $X$ be a compact topological space, $\mathcal{A}$ an algebra of subsets of $X, H$ a Hilbert space, and $Q: \mathcal{A} \rightarrow \mathcal{B}(H)$ be such that operators $Q(A)$ are mutually commuting Hermitian projections, $Q(A \cup B)=Q(A)+Q(B)$ whenever $A \cap B=\emptyset, A, B \in \mathcal{A}$. Assume that for each $x \in H$ the function $\mathcal{A} \in A \mapsto\langle Q(A) x, x\rangle \in[0, \infty)$ satisfies the regularity condition 2.4. (a). Then $Q$ has a unique extension to a projection-valued measure on the $\sigma$-field generated by $\mathcal{A}$.

Proof. We choose $x \in H$ such that $\langle P x, x\rangle>0$ for every non-zero projection $P$ belonging to the set $\mathcal{P}$ of all Hermitian projections of the commutative von Neumann algebra generated by $\{Q(A): A \in \mathcal{A}\}$. We apply Lemma 2.4 to the function $\mu: \mathcal{A} \rightarrow[0, \infty), \mu(A)=\langle Q(A) x, x\rangle$. Let $\mathcal{A}$ be equipped with the metric $\operatorname{dist}(A, B)=\mu(A \triangle B)$, and $\mathcal{P}$ - with the metric $\operatorname{dist}(P, Q)=$ $\langle|P-Q| x, x\rangle$, then $Q: \mathcal{A} \rightarrow \mathcal{P}$ is isometric, and can be extended by continuity to the $\sigma$-field generated by $\mathcal{A}$.

We return to $\left(H_{s, t}\right)_{s<t}$ and $Q_{E}$. The increasing projection-valued function $t \mapsto Q_{(0, t)}$ has at most a countable set of discontinuity points (since $H_{0,1}$ is separable), as well as the decreasing $t \mapsto Q_{(t, 1)}$. We denote by $D$ the union of these two sets, and restrict ourselves to elementary sets (2.1) such that $s_{1}, t_{1}, \ldots, s_{n}, t_{n} \notin D$ - these will be called regular elementary sets. [0 Note that continuity (at $\varepsilon=0+$ ) of $Q_{(0, t-\varepsilon)}=\left(\mathbf{1}_{0, t-\varepsilon} \otimes P_{t-\varepsilon, t}^{\prime}\right) \otimes P_{t, 1}^{\prime}$ implies continuity of $\mathbf{1}_{0, t-\varepsilon} \otimes P_{t-\varepsilon, t}^{\prime}$; similarly, continuity of $Q_{(t+\varepsilon, 1)}=P_{0, t}^{\prime} \otimes\left(P_{t, t+\varepsilon}^{\prime} \otimes\right.$ $\left.\mathbf{1}_{t+\varepsilon, 1}\right)$ implies continuity of $P_{t, t+\varepsilon}^{\prime} \otimes \mathbf{1}_{t+\varepsilon, 1}$. Together, they imply continuity of $\left(\mathbf{1}_{0, t-\varepsilon} \otimes P_{t-\varepsilon, t}^{\prime}\right) \otimes\left(P_{t, t+\varepsilon}^{\prime} \otimes \mathbf{1}_{t+\varepsilon, 1}\right)=Q_{(0, t-\varepsilon) \cup(t+\varepsilon, 1)}$ provided that $t \in(0,1) \backslash D$. It follows easily that $Q_{E}$ treated as a function of $2 n$ variables $s_{1}, t_{1}, \ldots, s_{n}, t_{n}$ (according to (2.1) ) is continuous in these variables at every point such that $s_{1}, t_{1}, \ldots, s_{n}, t_{n} \in(0,1) \backslash D$.

We introduce topological space $\mathcal{C}$ of all closed sets $C \subset[0,1]$, equipped with the Vietoris topology, that is, the Hausdorff metric

$$
\operatorname{dist}\left(C_{1}, C_{2}\right)=\max _{t \in[0,1]}\left|\min _{s \in C_{1}}\right| s-t\left|-\min _{s \in C_{2}}\right| s-t||
$$

under the convention that $\min _{s \in \emptyset}|s-t|=1$ for all $t$. It is well-known that $\mathcal{C}$ is compact.

We denote by $\mathcal{A}_{0}$ the algebra of subsets of $\mathcal{C}$ generated by subsets of the form $\{C \in \mathcal{C}: C \cap I \neq \emptyset\}$, where $I$ runs over intervals $I \subset[0,1]$ of all kinds (say, $(s, t)$, and $[s, t)$, and $[t, t]=\{t\}$, and $\emptyset$, etc). Every partition $[0,1]=I_{1} \cup \cdots \cup I_{n}$ of $[0,1]$ into a finite number $n$ of intervals $I_{1}, \ldots, I_{n}$ leads

\footnotetext{
${ }^{6}$ In the homogeneous setup, the set $D$ is necessarily empty; all elementary sets are regular. The reader may restrict himself to such a case.
} 
to a partition of $\mathcal{C}$ into $2^{n}$ sets of $\mathcal{A}_{0}$ (just choose for each $k=1, \ldots, n$ one of two possibilities, either $C \cap I_{k}=\emptyset$ or $C \cap I_{k} \neq \emptyset$ ), that is, a finite subalgebra of $\mathcal{A}_{0}$ containing $2^{2^{n}}$ sets.

A finer partition of $[0,1]$ leads to a finer partition of $\mathcal{C}$ and a larger finite subalgebra of $\mathcal{A}_{0}$. The whole $\mathcal{A}_{0}$ is the union of these finite subalgebras (over all partitions of $[0,1])$.

However, we restrict ourselves to 'regular' intervals, whose endpoints belong to $(0,1) \backslash D$ (though, intervals $[0, t),[0, t],(t, 1]$ and $[t, 1]$ are regular for $t \in(0,1) \backslash D)$, and the corresponding 'regular' subalgebra $\mathcal{A} \subset \mathcal{A}_{0}$, the union of $2^{2^{n}}$-subalgebras over all 'regular' partitions.

We may define a projection-valued additive function $\mu: \mathcal{A} \rightarrow \mathcal{B}\left(H_{0,1}\right)$ by

$$
\mu(\{C: C \cap I=\emptyset\})=Q_{[0,1] \backslash I}
$$

for any 'regular' interval $I \subset[0,1]$. Indeed, each of the $2^{n}$ elements of the partition of $\mathcal{C}$ that correspond to $[0,1]=I_{1} \cup \cdots \cup I_{n}$ is the intersection of $n$ sets of the form $\left\{C: C \cap I_{k}=\emptyset\right\}$ or $\left\{C: C \cap I_{k} \neq \emptyset\right\}$, and the corresponding projection is the product of $n$ projections of the form $Q_{[0,1] \backslash I_{k}}$ or $\mathbf{1}-Q_{[0,1] \backslash I_{k}}$.

It follows from (2.7) (using multiplicativity) that

$$
\mu(\{C: C \subset E\})=Q_{E}
$$

for any 'regular' elementary set $E$.

2.9 Lemma. The additive function $\mu$ has a unique extension to a projection measure on the Borel $\sigma$-field of $\mathcal{C}$.

Proof. The $2^{n}$ elements of the partition of $\mathcal{C}$ that correspond to $[0,1]=$ $I_{1} \cup \cdots \cup I_{n}$ are sets of diameter $\leq \max \left(\left|I_{1}\right|, \ldots,\left|I_{n}\right|\right)$. It follows easily that the $\sigma$-field generated by $\mathcal{A}$ is the whole Borel $\sigma$-field of $\mathcal{C}$. Due to Lemma 2.6, it is enough to check the regularity condition for $\langle\mu(A) x, x\rangle$. Due to Remark 2.5 we may do it only for $A=\{C: C \cap I=\emptyset\}$ and its complement. It remains to note that $Q_{[0, s] \cup[t, 1]}$ is continuous in $s, t$ whenever $s, t \in(0,1) \backslash D$; the same for $Q_{[0, s]}$ and $Q_{[t, 1]}$.

The projection-valued Borel measure $\mu$ on $\mathcal{C}$ that satisfies (2.7), (2.8), will be called the spectral measure of the pair $\left(H_{s, t}\right)_{s<t},\left(H_{s, t}^{\prime}\right)_{s<t}$.

For every $x \in H_{0,1}$, the positive Borel measure $A \mapsto\langle\mu(A) x, x\rangle$ will be called the spectral measure of $x$.

The $\sigma$-ideal of Borel sets $A_{0} \subset \mathcal{C}$ such that $\mu\left(A_{0}\right)=0$, or equivalently, the $\sigma$-filter of Borel sets $A_{1} \subset \mathcal{C}$ such that $\mu\left(\mathcal{C} \backslash A_{1}\right)=0$, is an invariant of the 
pair $\left(H_{s, t}\right)_{s<t},\left(H_{s, t}^{\prime}\right)_{s<t}$. It is convenient to express the relation $\mu\left(\mathcal{C} \backslash A_{1}\right)=0$ by saying that 'almost all spectral sets belong to $A_{1}$ '.

Note that

$$
\mu(\{C: C \ni t\})=0 \quad \text { for each } t \in(0,1) \backslash D ;
$$

in other words, almost all spectral sets do not contain a given point $t$ (unless $t$ belongs to at most countable set $D$ ). It follows (via Fubini's theorem) that almost all spectral sets are of zero Lebesgue measure, and therefore, nowhere dense.

Till now, Hilbert spaces over $\mathbb{C}$ were considered. However, some constructions are more natural over $\mathbb{R}$. Definition 1.5 works equally well over $\mathbb{R}$, giving 'real CP HS'. Every Hilbert space $H$ over $\mathbb{R}$ ('real Hilbert space') has its complexification $H^{\mathbb{C}}$, a Hilbert space over $\mathbb{C}$ ('complex Hilbert space') containing $H$ as an $\mathbb{R}$-linear subspace. Every $A \in \mathcal{B}(H)$ has its complexification $A^{\mathbb{C}} \in \mathcal{B}\left(H^{\mathbb{C}}\right)$; we have $\left\|A^{\mathbb{C}}\right\|=\|A\|,\left(A^{*}\right)^{\mathbb{C}}=\left(A^{\mathbb{C}}\right)^{*},(A B)^{\mathbb{C}}=A^{\mathbb{C}} B^{\mathbb{C}}$. On the other hand, the general form of an element $A \in \mathcal{B}\left(H^{\mathbb{C}}\right)$ is $A=$ $(\operatorname{Re} A)^{\mathbb{C}}+i(\operatorname{Im} A)^{\mathbb{C}} ; \operatorname{Re} A, \operatorname{Im} A \in \mathcal{B}(H) . \square$ Note that $\max (\|\operatorname{Re} A\|,\|\operatorname{Im} A\|) \leq$ $\|A\| \leq\|\operatorname{Re} A\|+\|\operatorname{Im} A\|$. Every real unitary (that is, invertible linear isometric) operator $U: H_{1} \rightarrow H_{2}$ between real Hilbert spaces has its complexification $U^{\mathbb{C}}: H_{1}^{\mathbb{C}} \rightarrow H_{2}^{\mathbb{C}}$, a complex unitary operator. Compositions are respected: $\left(U_{2} U_{1}\right)^{\mathbb{C}}=U_{2}^{\mathbb{C}} U_{1}^{\mathbb{C}}$. Tensor products are also respected: $\left(H_{1} \otimes H_{2}\right)^{\mathbb{C}}=$ $H_{1}^{\mathbb{C}} \otimes H_{2}^{\mathbb{C}}$ (up to a canonical isomorphism), and $(A \otimes B)^{\mathbb{C}}=A^{\mathbb{C}} \otimes B^{\mathbb{C}}$. Thus, every real CP HS $\left(\left(H_{s, t}\right)_{s<t},\left(W_{r, s, t}\right)_{r<s<t}\right)$ has its complexification $\left(\left(H_{s, t}^{\mathbb{C}}\right)_{s<t},\left(W_{r, s, t}^{\mathbb{C}}\right)_{r<s<t}\right)$, a complex CP HS. Isomorphic real CPs HS have isomorphic complexifications.

Can it happen that two nonisomorphic real CPs HS have isomorphic complexifications? I do not know.

However, invariants introduced in this section are robust to complexification, as stated below.

Definition 2.2 works equally well over $\mathbb{R}$.

2.11 Lemma. A sequence of elementary sets is infinitesimal for a real CP HS if and only if it is infinitesimal for the complexified CP HS. (The time set is $[0,1]$, as before.)

Proof. We have $H_{0,1}=H_{E_{k}} \otimes H_{(0,1) \backslash E_{k}}, \mathcal{A}_{k}=\mathcal{B}\left(H_{E_{k}}\right) \otimes \mathbf{1}_{(0,1) \backslash E_{k}}$, and $H_{0,1}^{\mathbb{C}}=H_{E_{k}}^{\mathbb{C}} \otimes H_{(0,1) \backslash E_{k}}^{\mathbb{C}}, \mathcal{A}_{k}^{\mathbb{C}}=\mathcal{B}\left(H_{E_{k}}^{\mathbb{C}}\right) \otimes \mathbf{1}_{(0,1) \backslash E_{k}}$. The general form of an element $A \in \mathcal{A}_{k}^{\mathbb{C}}$ is $A=(\operatorname{Re} A)^{\mathbb{C}}+i(\operatorname{Im} A)^{\mathbb{C}} ; \operatorname{Re} A, \operatorname{Im} A \in \mathcal{A}_{k}$;

\footnotetext{
${ }^{7}$ Here $\operatorname{Re} A$ is not $\left(A+A^{*}\right) / 2$. Rather, it may be thought of as the component-wise real part of a matrix.
} 
$\max (\|\operatorname{Re} A\|,\|\operatorname{Im} A\|) \leq\|A\| \leq\|\operatorname{Re} A\|+\|\operatorname{Im} A\|$. Similarly, the general form of a trace-class operator $R \in \mathcal{B}\left(H_{0,1}^{\mathbb{C}}\right)$ is $(\operatorname{Re} R)^{\mathbb{C}}+i(\operatorname{Im} R)^{\mathbb{C}}$, where $\operatorname{Re} R, \operatorname{Im} R \in \mathcal{B}\left(H_{0,1}\right)$ are trace-class operators, and $\operatorname{tr}(R)=$ $\operatorname{tr}(\operatorname{Re} R)+i \operatorname{tr}(\operatorname{Im} R)$. Thus, $A R=\left((\operatorname{Re} A)^{\mathbb{C}}+i(\operatorname{Im} A)^{\mathbb{C}}\right)\left((\operatorname{Re} R)^{\mathbb{C}}+\right.$ $\left.i(\operatorname{Im} R)^{\mathbb{C}}\right)=((\operatorname{Re} A)(\operatorname{Re} R))^{\mathbb{C}}-((\operatorname{Im} A)(\operatorname{Im} R))^{\mathbb{C}}+i((\operatorname{Re} A)(\operatorname{Im} R))^{\mathbb{C}}+$ $i((\operatorname{Im} A)(\operatorname{Re} R))^{\mathbb{C}} ; \quad \operatorname{tr}\left(((\operatorname{Re} A)(\operatorname{Re} R))^{\mathbb{C}}\right)=\operatorname{tr}((\operatorname{Re} A)(\operatorname{Re} R)), \quad$ etc.; $|\operatorname{tr}(A R)| \leq|\operatorname{tr}((\operatorname{Re} A)(\operatorname{Re} R))|+($ three more terms $)$;

$$
\sup _{A \in \mathcal{A}_{k}^{\mathbb{C}},\|A\| \leq 1}|\operatorname{tr}(A R)| \leq 2 \sup _{A \in \mathcal{A}_{k},\|A\| \leq 1}|\operatorname{tr}(A \operatorname{Re} R)|+2 \sup _{A \in \mathcal{A}_{k},\|A\| \leq 1}|\operatorname{tr}(A \operatorname{Im} R)| .
$$

If the sequence is infinitesimal for the real CP HS, then the right-hand side tends to 0 , and therefore the left-hand side tends to 0 , which means that the sequence is infinitesimal for the complexified CP HS.

On the other hand, if $R \in \mathcal{B}\left(H_{0,1}\right)$ is a trace-class operator, then $\operatorname{tr}(A R)=$ $\operatorname{tr}\left(A^{\mathbb{C}} R^{\mathbb{C}}\right)$ for $A \in \mathcal{A}_{k}$, and

$$
\sup _{A \in \mathcal{A}_{k},\|A\| \leq 1}|\operatorname{tr}(A R)| \leq \sup _{A \in \mathcal{A}_{k}^{\mathbb{C}},\|A\| \leq 1}\left|\operatorname{tr}\left(A R^{\mathbb{C}}\right)\right|
$$

which gives us the second implication.

We turn to a pair, a CP HS $\left(H_{s, t}\right)_{s<t}$ on $[0,1]$ and an embedded CP HS $\left(H_{s, t}^{\prime}\right)_{s<t}, H_{s, t}^{\prime} \subset H_{s, t}$, assuming this time, that $H_{s, t}, H_{s, t}^{\prime}$ are real (rather than complex). Projections $Q_{E}$ are still well-defined, and the spectral measure $\mu$ satisfying (2.7), (2.8) emerges as before. Alternatively we may consider the complex embedding $H_{s, t}^{\prime \mathbb{C}} \subset H_{s, t}^{\mathbb{C}}$. Corresponding projections are just $Q_{E}^{\mathbb{C}}$ (since $\left.\left(\left(H / H^{\prime}\right)_{E}\right)^{\mathbb{C}}=\left(H^{\mathbb{C}} / H^{\prime \mathbb{C}}\right)_{E}\right)$. It follows that the spectral measure for the complex embedding is the complexification of $\mu$, that is, $A \mapsto(\mu(A))^{\mathbb{C}}$ (here $(\mu(A))^{\mathbb{C}}$ is the complexification of the projection $\mu(A)$ ). Indeed, (2.8) shows that the equality between the two projection-valued Borel measures holds on sets of the form $A=\{C: C \subset E\}$; therefore it holds for all sets. The conclusion follows.

2.12 Lemma. The complexification $A \mapsto(\mu(A))^{\mathbb{C}}$ of the spectral measure $\mu$ of an embedding $H_{s, t}^{\prime} \subset H_{s, t}$ of real CPs HS is equal to the spectral measure of the corresponding embedding $H_{s, t}^{\mathbb{C}} \subset H_{s, t}^{\mathbb{C}}$ of complex CPs HS.

All said in sections 3-7 holds for real and complex Hilbert spaces equally well. 


\section{Continuous products of measure classes}

'Square roots of measures', introduced by Accardi [Ac76], are quite useful for constructing product systems, which was suggested by A. Vershik. For definitions and basic facts see [A], Sect. 3.3.5], [Aㄱ, Sect. 14.4]. The following definition is somewhat more restrictive than Arveson's, since I restrict myself to measure classes generated by a single measure.

3.1 Definition. (a) A measure class is a triple $(X, \mathcal{B}, \mathcal{M})$ consisting of a standard Borel space $(X, \mathcal{B})$ and a nonempty set $\mathcal{M}$ of finite positive measures on $(X, \mathcal{B})$ such that for some (therefore, every) $\mu \in \mathcal{M}$ and for every finite positive measure $\nu$ on $(X, \mathcal{B})$,

$$
\nu \sim \mu \text { if and only if } \nu \in \mathcal{M},
$$

$\nu \sim \mu$ denoting mutual absolute continuity. (Such $\mathcal{M}$ will be called an equivalence class of measures.)

(b) An isomorphism of a measure class $(X, \mathcal{B}, \mathcal{M})$ to another measure class $\left(X^{\prime}, \mathcal{B}^{\prime}, \mathcal{M}^{\prime}\right)$ is a map $\eta: X \rightarrow X^{\prime}$ which is a $\bmod 0$ isomorphism of measure spaces $(X, \mathcal{B}, \mu)$ and $\left(X^{\prime}, \mathcal{B}^{\prime}, \mu^{\prime}\right)$ for some $\mu \in \mathcal{M}, \mu^{\prime} \in \mathcal{M}^{\prime}$.

As usual, all negligible sets (that is, subsets of Borel sets of measure 0 for some, therefore every, $\mu \in \mathcal{M}$ ) may be added to the $\sigma$-field. Instead of requiring $(X, \mathcal{B})$ to be a standard Borel space, we may require for some (therefore every) $\mu \in \mathcal{M}$, that $(X, \mathcal{B}, \mu)$ is a Lebesgue-Rokhlin measure space; it means a measure space, isomorphic mod0 to some interval $(0, a)$ with Lebesgue measure, or a discrete (finite or countable) measure space, or a combination of both. Up to mod0 isomorphism, the two approaches are equivalent.

Throughout, either by assumption or by construction, all measure spaces will be Lebesgue-Rokhlin spaces;flalso, all claims and constructions will be invariant under mod 0 isomorphisms.

Hilbert spaces $L_{2}(\mu), L_{2}(\nu)$ for $\mu, \nu \in \mathcal{M}$ may be glued together via the unitary operator

$$
L_{2}(\mu) \ni f \mapsto \sqrt{\frac{\mu}{\nu}} f \in L_{2}(\nu) ;
$$

here $f$ is the Radon-Nikodym derivative (denoted also by $\frac{d \mu}{d \nu}$ ). These spaces may be treated as 'incarnations' of a single Hilbert space $L_{2}(X, \mathcal{B}, \mathcal{M})$.'t

\footnotetext{
${ }^{8}$ Another restriction in addition to Arveson's.

${ }^{9}$ In particular, all negligible sets are measurable.

${ }^{10}$ More formally: an element of $L_{2}(X, \mathcal{B}, \mathcal{M})$ is, by definition, a family $\psi=\left(\psi_{\mu}\right)_{\mu \in \mathcal{M}}$ such that $\psi_{\mu} \in L_{2}(X, \mathcal{B}, \mu)$ for every $\mu \in \mathcal{M}$, and $\psi_{\nu}=\sqrt{\frac{\underline{\mu}}{\nu}} \psi_{\mu}$ for all $\mu, \nu \in \mathcal{M}$.
} 
The general form of an element of $L_{2}(X, \mathcal{B}, \mathcal{M})$ is $f \sqrt{\mu}$, where $\mu \in \mathcal{M}$ and $f \in L_{2}(X, \mathcal{B}, \mu)$, taking into account the relation

$$
f \sqrt{\mu}=\left(\sqrt{\frac{\mu}{\nu}} f\right) \sqrt{\nu} .
$$

Any isomorphism of measure classes induces naturally a unitary operator between the corresponding Hilbert spaces.

The product of two measure classes is defined naturally, and

$$
L_{2}\left((X, \mathcal{B}, \mathcal{M}) \times\left(X^{\prime}, \mathcal{B}^{\prime}, \mathcal{M}^{\prime}\right)\right)=L_{2}(X, \mathcal{B}, \mathcal{M}) \otimes L_{2}\left(X^{\prime}, \mathcal{B}^{\prime}, \mathcal{M}^{\prime}\right)
$$

that is, we have a canonical unitary operator between these spaces, namely, $f \sqrt{\mu} \otimes f^{\prime} \sqrt{\mu^{\prime}} \mapsto\left(f \otimes f^{\prime}\right) \sqrt{\mu \otimes \mu^{\prime}}$, where $\left(f \otimes f^{\prime}\right)\left(\omega, \omega^{\prime}\right)=f(\omega) f^{\prime}\left(\omega^{\prime}\right)$. I'll write in short $L_{2}(\mathcal{M})$ instead of $L_{2}(X, \mathcal{B}, \mathcal{M})$; thus, $L_{2}\left(\mathcal{M} \times \mathcal{M}^{\prime}\right)=L_{2}(\mathcal{M}) \otimes$ $L_{2}\left(\mathcal{M}^{\prime}\right)$. Everyone knows the similar fact for measure spaces, $L_{2}\left(\mu \times \mu^{\prime}\right)=$ $L_{2}(\mu) \otimes L_{2}\left(\mu^{\prime}\right)$.

Given isomorphisms of measure classes $\eta: \mathcal{M} \rightarrow \mathcal{M}_{1}$ (that is, $\eta$ : $\left.(X, \mathcal{B}, \mathcal{M}) \rightarrow\left(X_{1}, \mathcal{B}_{1}, \mathcal{M}_{1}\right)\right)$ and $\eta^{\prime}: \mathcal{M}^{\prime} \rightarrow \mathcal{M}_{1}^{\prime}$, the map $\eta \times \eta^{\prime}$ defined by $\left(\eta \times \eta^{\prime}\right)\left(\omega, \omega^{\prime}\right)=\left(\eta(\omega), \eta^{\prime}\left(\omega^{\prime}\right)\right)$, is an isomorphism of measure classes, $\eta \times \eta^{\prime}: \mathcal{M} \times \mathcal{M}^{\prime} \rightarrow \mathcal{M}_{1} \times \mathcal{M}_{1}^{\prime}$.

3.2 Definition. (a) A continuous product of measure classes (' $\mathrm{CP} M C$ ', for short) is a family $\left(\left(X_{s, t}, \mathcal{B}_{s, t}, \mathcal{M}_{s, t}\right)\right)_{-\infty \leq s<t \leq \infty}$ of measure classes such that

$$
\left(X_{r, t}, \mathcal{B}_{r, t}, \mathcal{M}_{r, t}\right)=\left(X_{r, s}, \mathcal{B}_{r, s}, \mathcal{M}_{r, s}\right) \times\left(X_{s, t}, \mathcal{B}_{s, t}, \mathcal{M}_{s, t}\right)
$$

whenever $-\infty \leq r<s<t \leq \infty$.

(b) An isomorphism of one $\mathrm{CP} \operatorname{MC}\left(\left(X_{s, t}, \mathcal{B}_{s, t}, \mathcal{M}_{s, t}\right)\right)_{-\infty \leq s<t \leq \infty}$ to another $\mathrm{CP} \operatorname{MC}\left(\left(X_{s, t}^{\prime}, \mathcal{B}_{s, t}^{\prime}, \mathcal{M}_{s, t}^{\prime}\right)\right)_{-\infty \leq s<t \leq \infty}$ is a family $\left(\eta_{s, t}\right)_{-\infty \leq s<t \leq \infty}$ where each $\eta_{s, t}$ is an isomorphism of measure classes, $\left(X_{s, t}, \mathcal{B}_{s, t}, \mathcal{M}_{s, t}\right)$ to $\left(X_{s, t}^{\prime}, \mathcal{B}_{s, t}^{\prime}, \mathcal{M}_{s, t}^{\prime}\right)$, and

$$
\eta_{r, t}=\eta_{r, s} \times \eta_{s, t}
$$

whenever $-\infty \leq r<s<t \leq \infty$.

I'll write simply $\left(\mathcal{M}_{s, t}\right)_{s<t}$ instead of $\left(\left(X_{s, t}, \mathcal{B}_{s, t}, \mathcal{M}_{s, t}\right)\right)_{s<t}$. Given a CP $\operatorname{MC}\left(\mathcal{M}_{s, t}\right)_{s<t}$, we may construct the corresponding CP HS $\left(H_{s, t}\right)_{s<t}$; just $H_{s, t}=L_{2}\left(\mathcal{M}_{s, t}\right)$.

Does every CP HS (up to isomorphism) emerge from some CP MC? I do not know.

All product systems constructed in this paper emerge (via CPs HS) from CPs MC. 
Another useful language for describing CPs MC is based on sub- $\sigma$-fields. By a sub- $\sigma$-field on a (Lebesgue-Rokhlin!) measure space $(X, \mathcal{B}, \mu)$, or a measure class space $(X, \mathcal{B}, \mathcal{M})$, I always mean a sub- $\sigma$-field of $\mathcal{B}$ that contains all negligible sets. The sub- $\sigma$-field generated by the union $\mathcal{F}_{1} \cup \mathcal{F}_{2}$ of sub- $\sigma$ fields $\mathcal{F}_{1}, \mathcal{F}_{2}$ will be denoted by $\mathcal{F}_{1} \vee \mathcal{F}_{2}$.

3.3 Definition. Sub- $\sigma$-fields $\mathcal{F}_{1}, \ldots, \mathcal{F}_{n}$ on a measure class $(X, \mathcal{B}, \mathcal{M})$ are independent, $\square$ if there exists a probability measure $\mu \in \mathcal{M}$ such that

$$
\mu\left(A_{1} \cap \cdots \cap A_{n}\right)=\mu\left(A_{1}\right) \ldots \mu\left(A_{n}\right) \quad \text { for all } A_{1} \in \mathcal{F}_{1}, \ldots, A_{n} \in \mathcal{F}_{n} .
$$

For independent $\mathcal{F}_{1}, \ldots, \mathcal{F}_{n}$ the sub- $\sigma$-field $\mathcal{F}_{1} \vee \cdots \vee \mathcal{F}_{n}$ generated by them will be denoted also by $\mathcal{F}_{1} \otimes \ldots \otimes \mathcal{F}_{n}$.

Given a product of two measure classes,

$$
(X, \mathcal{B}, \mathcal{M})=\left(X_{1}, \mathcal{B}_{1}, \mathcal{M}_{1}\right) \times\left(X_{2}, \mathcal{B}_{2}, \mathcal{M}_{2}\right),
$$

we have two independent sub- $\sigma$-fields $\mathcal{F}_{1}, \mathcal{F}_{2}$ such that $\mathcal{B}=\mathcal{F}_{1} \otimes \mathcal{F}_{2}$; roughly,

$$
\mathcal{F}_{1}=\left\{A \times X_{2}: A \in \mathcal{B}_{1}\right\}, \quad \mathcal{F}_{2}=\left\{X_{1} \times B: B \in \mathcal{B}_{2}\right\},
$$

though, all negligible sets must be added.

And conversely, every two independent sub- $\sigma$-fields $\mathcal{F}_{1}, \mathcal{F}_{2}$ such that $\mathcal{B}=$ $\mathcal{F}_{1} \otimes \mathcal{F}_{2}$ emerge from a representation of $(X, \mathcal{B}, \mathcal{M})$ (up to isomorphism) as a product; in fact, $\left(X_{k}, \mathcal{B}_{k}, \mathcal{M}_{k}\right)=(X, \mathcal{B}, \mathcal{M}) / \mathcal{F}_{k}$ is the quotient space.

3.5 Remark. If $\mathcal{F}_{1}, \mathcal{F}_{2}, \mathcal{F}_{3}$ are sub- $\sigma$-fields on a measure class $(X, \mathcal{B}, \mathcal{M})$ such that

$$
\begin{gathered}
\mathcal{F}_{1} \text { and }\left(\mathcal{F}_{2} \vee \mathcal{F}_{3}\right) \text { are independent, } \\
\mathcal{F}_{2} \text { and } \mathcal{F}_{3} \text { are independent, }
\end{gathered}
$$

then

$$
\mathcal{F}_{1}, \mathcal{F}_{2}, \mathcal{F}_{3} \text { are independent. }
$$

Indeed, we can split off the first factor, and then split the rest. (It may become a difficulty, if you do not use the technique of products.)

Given a $\mathrm{CP} \operatorname{MC}\left(\left(X_{s, t}, \mathcal{B}_{s, t}, \mathcal{M}_{s, t}\right)\right)_{-\infty \leq s<t \leq \infty}$, we have a family of sub- $\sigma$-fields $\left(\mathcal{F}_{s, t}\right)_{-\infty \leq s<t \leq \infty}$ on the measure class $(X, \mathcal{B}, \mathcal{M})=$ $\left(X_{-\infty, \infty}, \mathcal{B}_{-\infty, \infty}, \mathcal{M}_{-\infty, \infty}\right)$ satisfying $\mathcal{F}_{-\infty, \infty}=\mathcal{B}$ and

$$
\mathcal{F}_{r, t}=\mathcal{F}_{r, s} \otimes \mathcal{F}_{s, t} \quad \text { whenever }-\infty \leq s<t \leq \infty .
$$

\footnotetext{
${ }^{11}$ They could also be called quasiindependent; see footnotes 24, 28 .
} 
And conversely, every such family of sub- $\sigma$-fields on a measure class emerges from a CP MC.

Condition (3.6) is evidently equivalent to the combination of two conditions,

$$
\begin{gathered}
\mathcal{F}_{r, t}=\mathcal{F}_{r, s} \vee \mathcal{F}_{s, t} \quad \text { whenever }-\infty \leq r<s<t \leq \infty, \\
\mathcal{F}_{-\infty, s} \text { and } \mathcal{F}_{s, \infty} \text { are independent for all } s \in \mathbb{R} .
\end{gathered}
$$

3.9 Remark. It is tempting to restrict ourselves to one-parameter families $\left(\mathcal{F}_{-\infty, s}\right)_{s \in \mathbb{R}}$ and $\left(\mathcal{F}_{s, \infty}\right)_{s \in \mathbb{R}}$, since $\mathcal{F}_{s, t}=\mathcal{F}_{-\infty, s} \cap \mathcal{F}_{s, \infty}$. However, there is an obstacle, illustrated by the following elementary example. Let

$$
\begin{gathered}
X=\{-1,+1\}^{3}=\left\{\left(\tau_{1}, \tau_{2}, \tau_{3}\right): \tau_{1}, \tau_{2}, \tau_{3}= \pm 1\right\}, \\
\mathcal{B}=(\text { all subsets of } X), \\
\mu=(\text { the uniform probability distribution on } X), \\
\mathcal{F}_{1} \text { is generated by } \tau_{1}, \\
\mathcal{F}_{1,2} \text { is generated by } \tau_{1} \text { and }\left(1-\tau_{1}\right) \tau_{2}+\left(1+\tau_{1}\right) \tau_{2} \tau_{3}, \\
\mathcal{F}_{2,3} \text { is generated by } \tau_{2} \text { and } \tau_{3}, \\
\mathcal{F}_{3} \text { is generated by } \tau_{3} .
\end{gathered}
$$

Then $\mathcal{F}_{1} \subset \mathcal{F}_{1,2}, \mathcal{F}_{2,3} \supset \mathcal{F}_{3}$, and $\mathcal{F}_{1} \otimes \mathcal{F}_{2,3}=\mathcal{B}=\mathcal{F}_{1,2} \otimes \mathcal{F}_{3}$. Nevertheless, the sub- $\sigma$-field $\mathcal{F}_{2}=\mathcal{F}_{1,2} \cap \mathcal{F}_{2,3}$ is trivial, and so, $\mathcal{F}_{1} \vee \mathcal{F}_{2} \vee \mathcal{F}_{3}=\mathcal{F}_{1} \vee \mathcal{F}_{3} \neq \mathcal{B}$.

The following definition is equivalent to Definition 3.2.

3.10 Definition. (a) A CP MC is $\left((X, \mathcal{B}, \mathcal{M}),\left(\mathcal{F}_{s, t}\right)_{-\infty \leq s<t \leq \infty}\right)$ where $(X, \mathcal{B}, \mathcal{M})$ is a measure class and $\mathcal{F}_{s, t}$ are sub- $\sigma$-fields satisfying (3.7), (3.8) and $\mathcal{F}_{-\infty, \infty}=\mathcal{B}$.

(b) An isomorphism of one $\mathrm{CP} \mathrm{MC}\left((X, \mathcal{B}, \mathcal{M}),\left(\mathcal{F}_{s, t}\right)_{-\infty \leq s<t \leq \infty}\right)$ to another CP MC $\left(\left(X^{\prime}, \mathcal{B}^{\prime}, \mathcal{M}^{\prime}\right),\left(\mathcal{F}_{s, t}^{\prime}\right)_{-\infty \leq s<t \leq \infty}\right)$ is a mod 0 isomorphism of measure classes, $(X, \mathcal{B}, \mathcal{M})$ to $\left(X^{\prime}, \mathcal{B}^{\prime}, \mathcal{M}^{\prime}\right)$, that sends each $\mathcal{F}_{s, t}$ to $\mathcal{F}_{s, t}^{\prime}$.

3.11 Definition. A homogeneous continuous product of measure classes ('HCP MC', for short) is $\left((X, \mathcal{B}, \mathcal{M}),\left(\mathcal{F}_{s, t}\right)_{-\infty \leq s<t \leq \infty},\left(\tilde{\theta}^{u}\right)_{u \in \mathbb{R}}\right)$, where $\left((X, \mathcal{B}, \mathcal{M}),\left(\mathcal{F}_{s, t}\right)_{s<t}\right)$ is a CP MC, and $\left.\left(\tilde{\theta}^{u}\right)_{u \in \mathbb{R}}\right)$ is a one-parameter group of automorphisms $\tilde{\theta}^{u}$ of the measure class $(X, \mathcal{B}, \mathcal{M})$ such that

$$
\tilde{\theta}^{u}\left(\mathcal{F}_{s, t}\right)=\mathcal{F}_{s+u, t+u} \quad \text { whenever }-\infty \leq s<t \leq \infty, u \in \mathbb{R},
$$

and the corresponding unitary group $\left(\theta^{u}\right)_{u \in \mathbb{R}}$ on $L_{2}(X, \mathcal{B}, \mathcal{M})$ is strongly continuous.

I leave to the reader elaborating on the latter condition, and defining isomorphisms of HCPs MC.

Clearly, every HCP MC leads to an HCP HS, and (via Theorem 1.9) to a product system. 


\section{Continuous products of probability spaces}

By a probability space I always mean a (Lebesgue-Rokhlin!) measure space $(X, \mathcal{B}, \mu)$ such that $\mu(X)=1$. By an isomorphism of probability spaces I mean a mod0 isomorphism (measure preserving). The product of two probability spaces is a probability space, of course.

A continuous product of probability spaces ('CP PS', for short) is defined similarly to Def. 3.2(a); that is,

$$
\left(X_{r, t}, \mathcal{B}_{r, t}, \mu_{r, t}\right)=\left(X_{r, s}, \mathcal{B}_{r, s}, \mu_{r, s}\right) \times\left(X_{s, t}, \mathcal{B}_{s, t}, \mu_{s, t}\right),
$$

where $\mu_{s, t}$ are probability measures. Definition $3.2(\mathrm{~b})$ is also adapted readily.

An equivalent definition, similar to Definition 3.10: a CP PS is $((X, \mathcal{B}, \mu)$, $\left.\left(\mathcal{F}_{s, t}\right)_{-\infty \leq s<t \leq \infty}\right)$, where $(X, \mathcal{B}, \mu)$ is a probability space, and $\mathcal{F}_{s, t}$ are sub- $\sigma$ fields satisfying (3.6) (or equivalently, (3.7) and (3.8)); this time, independence means (3.4) for the given $\mu$ (rather than some equivalent measure). Definition 3.10(b) is also adapted readily.

Given a CP PS $\left(\mu_{s, t}\right)_{s<t}$, we may construct the corresponding CP HS $\left(H_{s, t}\right)_{s<t}$; just $H_{s, t}=L_{2}\left(\mu_{s, t}\right)$. Alternatively, we may construct first the corresponding $\mathrm{CP} \mathrm{MC}\left(\mathcal{M}_{s, t}\right)_{s<t}$, where $\mathcal{M}_{s, t}$ consists of all measures equivalent to $\mu_{s, t}$; and second, out of $\left(\mathcal{M}_{s, t}\right)_{s<t}$ we construct $\left(H_{s, t}\right)_{s<t}, H_{s, t}=L_{2}\left(\mathcal{M}_{s, t}\right)$. Both ways lead to the same (up to isomorphism) CP HS $\left(H_{s, t}\right)_{s<t}$. Thus, the class of CPs HS corresponding to CPs PS is a part of the class of CPs HS corresponding to CPs MC. It is a proper subclass. Indeed, any CP HS of the form $\left(L_{2}\left(\mu_{s, t}\right)\right)_{s<t}$ has a decomposable vector, namely, a constant function. (In fact, such a CP HS can be of type $I$ or $I I$.) In contrast, a CP HS of the form $\left(L_{2}\left(\mathcal{M}_{s, t}\right)\right)_{s<t}$ need not have a decomposable vector. (In fact, such a CP HS can be of type $I, I I$ or $I I I$.)

The lemma below establishes a relation between CPs MC, CPs PS and decomposable vectors. Note that for every measure class $(X, \mathcal{B}, \mathcal{M})$ and every vector $\psi \in L_{2}(\mathcal{M})$ we have the corresponding positive measure $|\psi|^{2}$ on $(X, \mathcal{B})$, satisfying $|\psi|^{2}(X)=\|\psi\|^{2}$. Namely, if $\psi=f \sqrt{\mu}$ then $|\psi|^{2}=|f|^{2} \mu$ (that is, $\frac{d|\psi|^{2}}{d \mu}=|f|^{2}$ ); the result does not depend on $\mu \in \mathcal{M}$. Also, for $\psi_{1} \in L_{2}\left(\mathcal{M}_{1}\right), \psi_{2} \in L_{2}\left(\mathcal{M}_{2}\right)$ we have $\left|\psi_{1} \otimes \psi_{2}\right|^{2}=\left|\psi_{1}\right|^{2} \times\left|\psi_{2}\right|^{2}$ (the product measure).

4.1 Lemma. Let $\left(\mathcal{M}_{s, t}\right)_{s<t}$ be a CP MC, and $\psi \in L_{2}\left(\mathcal{M}_{0,1}\right)$ a decomposable vector, $\|\psi\|=1 ; \psi=\psi_{0,1}, \psi_{r, t}=\psi_{r, s} \otimes \psi_{s, t}, \psi_{s, t} \in H_{s, t}=L_{2}\left(\mathcal{M}_{s, t}\right)$, $\left\|\psi_{s, t}\right\|=1$. Then measures $\mu_{s, t}=\left|\psi_{s, t}\right|^{2}$ form a CP PS $\left(\mu_{s, t}\right)_{s<t}$.

Proof. $\mu_{r, t}=\left|\psi_{r, t}\right|^{2}=\left|\psi_{r, s} \otimes \psi_{s, t}\right|^{2}=\left|\psi_{r, s}\right|^{2} \times\left|\psi_{s, t}\right|^{2}=\mu_{r, s} \times \mu_{s, t}$. 
Every CP PS $\left(\mu_{s, t}\right)_{s<t}$, be it of type $I$ or $I I$, can arise as $\mu_{s, t}=\left|\psi_{s, t}\right|^{2}$. Indeed, we may take the corresponding $\mathrm{CP} \mathrm{MC}\left(\mathcal{M}_{s, t}\right)_{s<t}$ and the decomposable vector $\psi_{s, t}=\sqrt{\mu_{s, t}}$.

Homogeneous CPs PS are defined evidently. HCPs PS, also called 'noises', are studied in probability theory, see a survey [ $\left.\Gamma_{\mathrm{s}}\right]$. Classical noises (white, Poisson and their combinations) lead to HCPs HS (and product systems) of type $I$. Nonclassical noises, found by J. Warren and S. Watanabe, lead to HCPs HS (and product systems) of type $I I_{n}, n>0$. Some of them have spectral sets of any given Hausdorff dimension between 0 and $1 / 2$. Some are time-asymmetric. Black noises, found by A. Vershik, myself and S. Watanabe, lead to HCPs HS (and product systems) of type $I I_{0}$. See $\Gamma_{\mathrm{s}}+$ for references and details. Thus, a part of the results of the present work can be derived from the theory of HCPs PS. However, HCPs MC give simpler proofs and stronger results about HCPs HS.

\section{Random sets, and type $I I_{0}$}

Let $\mathcal{C}$ be the set of all closed sets $C \subset \mathbb{R}$ (not just $C \subset[0,1]$ as in Sect. 2). We equip $\mathcal{C}$ with the Effros $\sigma$-field $\mathcal{B}$; it is generated by functions $C \mapsto$ $\min _{s \in C}|s-t|$ indexed by $t \in \mathbb{R}, \mathbb{E}$ and it turns $\mathcal{C}$ into a standard Borel space.

For any $s, t$ such that $-\infty \leq s<t \leq \infty$ we define the sub- $\sigma$-field $\mathcal{B}_{s, t} \subset \mathcal{B}$ generated by the map $C \mapsto C \cap[s, t]$, that is, by functions $C \mapsto$ $\min _{r \in C \cap[s, t]}|r-t|, t \in \mathbb{R}$. Clearly, these sub- $\sigma$-fields satisfy (3.7). Till now, no measure appears, and no sets are negligible. The next definition introduces an equivalence class $\mathcal{M}$ of measures on $\mathcal{C}$ (thus turning the Borel space $(\mathcal{C}, \mathcal{B})$ into a measure class $(\mathcal{C}, \mathcal{B}, \mathcal{M})$ ), after which all $\sigma$-fields must be completed with negligible sets.

5.1 Definition. An equivalence class $\mathcal{M}$ of measures on $(\mathcal{C}, \mathcal{B})$ is decomposable, if

(a) sub- $\sigma$-fields $\mathcal{B}_{-\infty, s}$ and $\mathcal{B}_{s, \infty}$ on the measure class $(\mathcal{C}, \mathcal{B}, \mathcal{M})$ are independent for all $s \in \mathbb{R}$;

(b) the set $\{C \in \mathcal{C}: C \ni t\}$ is negligible, for each $t \in \mathbb{R}$.

Condition (a) is just (3.8); we see that every decomposable equivalence class of measures $\mathcal{M}$ leads to a $\mathrm{CP} \mathrm{MC}\left((\mathcal{C}, \mathcal{B}, \mathcal{M}),\left(\mathcal{B}_{s, t}\right)_{-\infty \leq s<t \leq \infty}\right)$. The set $\{C \in \mathcal{C}: C \ni t\}$, belonging to the intersection $\mathcal{B}_{-\infty, t} \cap \mathcal{B}_{t, \infty}$ of independent sub- $\sigma$-fields, must be either negligible or co-negligible (that is, of negligible complement); the second possibility is of no interest for us, and we exclude it by (b).

\footnotetext{
${ }^{12}$ Once again, $\min _{s \in \emptyset}|s-t|=1$ for all $t$, by definition.
} 
Looking forward to a relation between $\mathcal{M}$ and spectral measures, I find it convenient to say 'almost all spectral sets belong to $A_{1}$ ' instead of ' $\mathcal{C} \backslash A_{1}$ is negligible (w.r.t. $\mathcal{M}$ )'. Similarly to Sect. 2 (recall (2.10)), almost all spectral sets do not contain a given point $t$ (due to 5.1(b)), therefore, almost all spectral sets are nowhere dense, of Lebesgue measure 0.

5.2 Lemma. Let $\mathcal{M}$ be a decomposable eqvivalence class of measures on $\mathcal{C}$, and $-\infty<s<t<\infty$. Then the set $\{C \in \mathcal{C}: C \cap[s, t]=\emptyset\}$ is not negligible.

Proof. Due to 5.1(b), each $r$ has a neighborhood $(r-\varepsilon, r+\varepsilon)$ such that the set $\{C \in \mathcal{C}: C \cap(r-\varepsilon, r+\varepsilon)=\emptyset\}$ is not negligible. Due to compactness of $[s, t]$ we may choose a single $\varepsilon$ for all $r \in[s, t]$. Non-negligible sets $\{C \in$ $\mathcal{C}: C \cap[s, s+\varepsilon]=\emptyset\}$ and $\{C \in \mathcal{C}: C \cap[s+\varepsilon, s+2 \varepsilon]=\emptyset\}$ belong to independent $\sigma$-fields $\mathcal{B}_{-\infty, s+\varepsilon}$ and $\mathcal{B}_{s+\varepsilon, \infty}$ respectively. Therefore their intersection $\{C \in \mathcal{C}: C \cap[s, s+2 \varepsilon]=\emptyset\}$ is not negligible. Continuing this way, we see that the set $\{C \in \mathcal{C}: C \cap[s, s+n \varepsilon] \cap[s, t]=\emptyset\}$ is not negligible for any $n$.

The quotient measure class $(\mathcal{C}, \mathcal{B}, \mathcal{M}) / \mathcal{B}_{s, t}$ may be thought of as the measure class $\left(\mathcal{C}_{s, t}, \tilde{\mathcal{B}}_{s, t}, \mathcal{M}_{s, t}\right)$, where $\mathcal{C}_{s, t}$ is the space of all closed subsets of $[s, t]$, $\tilde{\mathcal{B}}_{s, t}$ its Effros $\sigma$-field, and $\mathcal{M}_{s, t}$ consists of images of measures $\mu \in \mathcal{M}$ under the map $C \mapsto C \cap[s, t]$. Lemma 5.2 shows that the empty set (or rather, $\{\emptyset\}$ ) is an atom (non-negligible point) of $\left(\mathcal{C}_{s, t}, \tilde{\mathcal{B}}_{s, t}, \mathcal{M}_{s, t}\right)$. Therefore there exists a unique probability measure $\mu_{s, t} \in \mathcal{M}_{s, t}$ concentrated at the atom. Clearly, $\mu_{r, s} \times \mu_{s, t}=\mu_{r, t}$. In terms of the CP HS $\left(H_{s, t}\right)_{s<t}=\left(L_{2}\left(\mathcal{M}_{s, t}\right)\right)_{s<t}$ it means that vectors $\psi_{s, t}=\sqrt{\mu_{s, t}}$ satisfy

$$
\psi_{r, s} \otimes \psi_{s, t}=\psi_{r, t} \quad \text { whenever }-\infty<r<s<t<\infty .
$$

According to Definition 2.3(a), $\psi_{0,1}$ is a decomposable vector of the CP HS $\left(H_{s, t}\right)_{0 \leq s<t \leq 1}$ over $[0,1]$; let us call it 'the standard decomposable vector'. We see that each decomposable equivalence class $\mathcal{M}$ of measures on $\mathcal{C}$ leads to a CP HS of type $I$ or $I I$.

The spectral measure $\mu$ is defined (see Sect. 2) for the pair of the CP HS $\left(L_{2}\left(\mathcal{M}_{s, t}\right)\right)_{0 \leq s<t \leq 1}$ and its standard decomposable vector; $\mu$ is a projectionvalued Borel measure on $\left(\mathcal{C}_{0,1}, \tilde{\mathcal{B}}_{0,1}\right)$.

As every projection-valued measure, $\mu$ has its equivalence class $\mathcal{M}_{\mu}$ of measures; it consists of positive Borel measures on $\mathcal{C}_{0,1}$ of the form $A \mapsto$ $\langle\mu(A) \psi, \psi\rangle$, where $\psi$ runs over all vectors of $L_{2}\left(\mathcal{M}_{0,1}\right)$ such that $\langle\mu(A) \psi, \psi\rangle>$ 0 whenever $\mu(A) \neq 0$.

5.3 Lemma. $\mathcal{M}_{\mu}=\mathcal{M}$ for every decomposable eqvivalence class $\mathcal{M}$ of measures on $\mathcal{C}$. 
Proof. For any elementary set $E \subset(0,1)$, the subspace $\left(H / H^{\prime}\right)_{E}=Q_{E} H$ of $H=H_{0,1}$ may be described as consisting of vectors $\psi=f \sqrt{\mu}$ such that the measure $|\psi|^{2}=|f|^{2} \cdot \mu$ is concentrated on the set $\left\{C \in \mathcal{C}_{0,1}: C \subset E\right\}$. It means that $Q_{E}$ multiplies by the indicator of the set. Thus, for all $\psi$, $\left\langle Q_{E} \psi, \psi\right\rangle=|\psi|^{2}(\{C: C \subset E\})$. Using (2.8) we see that $\langle\mu(A) \psi, \psi\rangle=$ $|\psi|^{2}(A)$ for $A \subset \mathcal{C}_{0,1}$ of the form $\{C: C \subset E\}$; therefore it holds for all Borel sets $A$. Choosing $\psi$ such that $\langle\mu(A) \psi, \psi\rangle>0$ whenever $\mu(A) \neq 0$, we see that $|\psi|^{2} \in \mathcal{M}$. On the other hand, the measure $A \mapsto\langle\mu(A) \psi, \psi\rangle$ belongs to $\mathcal{M}_{\mu}$.

5.4 Remark. The spectral measure of an arbitrary vector $\psi \in H_{0,1}$ is given, for every Borel set $A \subset \mathcal{C}_{0,1}$, by $\langle\mu(A) \psi, \psi\rangle=|\psi|^{2}(A)$ (as is shown in the proof above).

5.5 Remark. We see that every 'random set' satisfying conditions 5.1 (a,b) can appear as the spectral set (of some pair of CPs HS). The converse is also true, with some reservations. Namely, let $\mu$ be the spectral measure of a pair ( $H_{s, t}^{\prime} \subset H_{s, t}$ for $\left.0 \leq s<t \leq 1\right)$, and $\mathcal{M}_{\mu}$ the corresponding equivalence class of measures on $\mathcal{C}_{0,1}$. Then $\mathcal{M}_{\mu}$ satisfies condition 5.1(b) for all $t \in(0,1) \backslash D$, where $D$ is an at most countable set (introduced in Sect. 2). Condition 5.1(a) is also satisfied for $s \in(0,1) \backslash D$. This fact will not be used, and I give only a hint toward the proof:

$$
\mu\left(A^{\prime} \times A^{\prime \prime}\right)=\mu^{\prime}\left(A^{\prime}\right) \otimes \mu^{\prime \prime}\left(A^{\prime \prime}\right)
$$

for all Borel sets $A^{\prime} \subset \mathcal{C}_{0, s}, A^{\prime \prime} \subset \mathcal{C}_{s, 1}$; here $\mu^{\prime}$ is the spectral measure of the restriction to $[0, s]$ of the given pair of CPs HS, thus, each $\mu^{\prime}\left(A^{\prime}\right)$ is a projection in $H_{0, s}$. Similarly, each $\mu^{\prime \prime}\left(A^{\prime \prime}\right)$ is a projection in $H_{s, 1}$.

A probability measure $\mu$ on $(\mathcal{C}, \mathcal{B})$ will be called decomposable, if

(a) sub- $\sigma$-fields $\mathcal{B}_{-\infty, s}$ and $\mathcal{B}_{s, \infty}$ on the probability space $(\mathcal{C}, \mathcal{B}, \mu)$ are independent for all $s \in \mathbb{R}$;

(b) [the same as 5.1(b)].

Such a measure $\mu$ leads to a $\operatorname{CP} \operatorname{PS}\left((\mathcal{C}, \mathcal{B}, \mu),\left(\mathcal{B}_{s, t}\right)_{-\infty \leq s<t \leq \infty}\right)$.

5.6 Lemma. A probability measure $\mu$ on $(\mathcal{C}, \mathcal{B})$ is decomposable if and only if it describes the Poisson point process corresponding to a nonatomic locally finite measure $\nu$ on $\mathbb{R}$.

Proof. 'If' is evident; 'only if' will be proven. Consider the number of points in a bounded interval, $N=|C \cap[s, t]|$ (an integer or infinity) as a random variable on $(\mathcal{C}, \mathcal{B}, \mu)$. By Lemma 5.2, $\mathbb{P}(N=0)>0$ (' $\mathbb{P}(\ldots)$ ' stands for probability). We divide $[s, t]$ into $n$ equal intervals ('cells') and consider the 
random number $N_{n}$ of occupied cells. Clearly, $N_{n} \uparrow N$ for $n \rightarrow \infty$. Due to independence, $\mathbb{P}\left(N_{n}=0\right)=\left(1-p_{1}^{(n)}\right) \ldots\left(1-p_{n}^{(n)}\right)$, where $p_{k}^{(n)}$ is the probability of cell $k$ being occupied. We know that $\inf _{n}\left(1-p_{1}^{(n)}\right) \ldots(1-$ $\left.p_{n}^{(n)}\right) \geq \mathbb{P}(N=0)>0$; therefore $\sup _{n} \mathbb{E} N_{n}=\sup _{n}\left(p_{1}^{(n)}+\cdots+p_{n}^{(n)}\right) \leq$ $-\ln \mathbb{P}(N=0)<\infty$. Also, $\max _{n}\left(p_{1}^{(n)}, \ldots, p_{n}^{(n)}\right) \rightarrow 0$ for $n \rightarrow \infty$ (recall the compactness argument in the proof of Lemma 5.2). By a well-known theorem of probability theory, $N$ has a Poisson distribution. It remains to use independence.

5.7 Corollary. Every decomposable probability measure on $(\mathcal{C}, \mathcal{B})$ is concentrated on the set of locally finite sets $C \in \mathcal{C}$ (that is, $C$ has no accumulation points).

A closed set $C \in \mathcal{C}$ is called perfect, if it has no isolated points. The only locally finite perfect set is the empty set.

5.8 Lemma. Let $\mathcal{M}$ be a decomposable eqvivalence class of measures on $\mathcal{C}$ such that almost all sets $C \in \mathcal{C}$ are perfect. Then the corresponding CP HS over $[0,1]$ is of type $I I_{0}$.

Proof. Let $\psi \in H_{0,1}$ be a decomposable vector. We have to prove that it is, up to a coefficient, the standard decomposable vector; that is, the measure $|\psi|^{2}$ is concentrated at the atom $C=\emptyset$. On one hand, the measure $|\psi|^{2}$, being absolutely continuous w.r.t. $\mathcal{M}_{0,1}$, is concentrated on (the set of all) perfect sets. Lemma 1.1), is concentrated on (the set of all) locally finite sets (by Corollary 5.7, adapted to $\left.\mathcal{C}_{0,1}\right)$.

5.9 Corollary. Let $\mathcal{M}, \mathcal{M}^{\prime}$ be decomposable equivalence classes of measures on $\mathcal{C}$, both such that almost all sets $C \in \mathcal{C}$ are perfect. Assume that their images $\mathcal{M}_{0,1}, \mathcal{M}_{0,1}^{\prime}$ under the map $C \mapsto C \cap[0,1]$ are different equivalence classes of measures on $\mathcal{C}_{0,1}$. Then the corresponding CPs HS $\left(L_{2}\left(\mathcal{M}_{s, t}\right)\right)_{0 \leq s<t \leq 1}$, $\left(L_{2}\left(\mathcal{M}_{s, t}^{\prime}\right)\right)_{0 \leq s<t \leq 1}$ over $[0,1]$ are non-isomorphic.

\section{Constructing random sets}

The set of zeros of a Brownian motion is a good example of a random set. More generally, we consider a stationary, sample-continuous, Fellerian Markov process $\left(\xi_{t}\right)_{-\infty<t<\infty}$ on a metrizable compact topological space $K$.

\footnotetext{
${ }^{13}$ If $C$ is a perfect set, then $C \cap[0,1]$ is perfect, provided that $0 \notin C, 1 \notin C$, which holds a.s.
} 
In other words, we have a strongly continuous semigroup $\left(T_{t}\right)_{0 \leq t<\infty}$ of positive unit-preserving operators $T_{t}$ on the space $C(K)$ of continuous functions $f: K \rightarrow \mathbb{R}$, the conjugate semigroup $\left(T_{t}^{*}\right)_{0 \leq t<\infty}$ on the space $M(K)$ of measures on $K$, and a probability measure $\nu$ on the space $C(\mathbb{R}, K)$ of continuous maps $\xi: \mathbb{R} \rightarrow K$ (called 'sample paths') such that

$$
\begin{aligned}
\int_{C(\mathbb{R}, K)} & f_{1}\left(\xi\left(t_{1}\right)\right) \ldots f_{n}\left(\xi\left(t_{n}\right)\right) d \nu(\xi)= \\
= & \int_{C(\mathbb{R}, K)} f_{1}\left(\xi\left(t_{1}\right)\right) \ldots f_{n-1}\left(\xi\left(t_{n-1}\right)\right)\left(T_{t_{n}-t_{n-1}} f_{n}\right)\left(\xi\left(t_{n-1}\right)\right) d \nu(\xi)
\end{aligned}
$$

whenever $n \in\{2,3, \ldots\}$ and $f_{1}, \ldots, f_{n} \in C(K)$. In more probabilistic language, the conditional distribution of $\xi\left(t_{n}\right)$ given $\xi\left(t_{1}\right), \ldots, \xi\left(t_{n-1}\right)$ is $T_{t_{n}-t_{n-1}}^{*} \delta_{\xi\left(t_{n-1}\right)}$; here $\delta_{a}$ is the unit mass at $a$. Note that the distribution of $\xi(0)$ (as well as any $\xi(t)$ ) is a probability measure $\nu_{0}$ on $K$, invariant in the sense that $T_{t}^{*} \nu_{0}=\nu_{0}$ for all $t$. The measure $\nu$ is uniquely determined by $\nu_{0}$ and $\left(T_{t}\right)_{0 \leq t<\infty}$, and is stationary (that is, invariant under time shifts).

Given a closed subset $K_{0} \subset K$ such that $\nu_{0}\left(K_{0}\right)=0$, we consider the random set $C=\left\{t \in \mathbb{R}: \xi(t) \in K_{0}\right\}$; it is a measurable map from the probability space $(C(\mathbb{R}, K), \nu)$ to the Borel space $\mathcal{C}$ of closed subsets of $\mathbb{R}$. The distribution of the random set is a probability measure $\mu$ on $\mathcal{C}$ that satisfies Condition 5.1(b), and is stationary. However, Condition 5.1(a) needs special effort.

Assume that the random set is non-empty a.s., and consider the first (after $t=0$ ) hit of $K_{0}$,

$$
\tau(\xi)=\min \left\{t \in[0, \infty): \xi(t) \in K_{0}\right\}
$$

the conditional distribution of the pair $(\tau, \xi(\tau))$, given $\xi(0)$, is a measurable map from $\left(K, \nu_{0}\right)$ to probability measures on $[0, \infty) \times K_{0}$.

The first condition of smearing. There exists an equivalence class of measures on $[0, \infty) \times K_{0}$ that contains almost all conditional distributions of $(\tau, \xi(\tau))$ given $\xi(0)$. (That is, for $\nu_{0}$-almost all $a \in K$ the conditional distribution of $(\tau, \xi(\tau))$ given $\xi(0)=a$ belongs to the equivalence class.)

Note that $\{0\} \times K_{0}$ is negligible in $[0, \infty) \times K_{0}$, since $\xi(0) \notin K_{0}$ a.s.

\footnotetext{
${ }^{14}$ Existence of an invariant measure $\nu_{0}$ for a given semigroup follows easily from compactness; namely, $\left\|\left(T_{t}^{*}-\mathbf{1}\right) \frac{1}{s} \int_{0}^{s} T_{r}^{*} d r\right\| \leq \frac{2 t}{s} \rightarrow 0$ for $s \rightarrow \infty$, therefore each weak ${ }^{*}$-limit point (for $s \rightarrow \infty$ ) of $\frac{1}{s} \int_{0}^{s}\left(T_{r}^{*}\right)\left(\nu_{1}\right) d r$ is an invariant measure, irrespective of the choice a probability measure $\nu_{1}$. However, we do not need it.
} 
6.2 Lemma. The first condition of smearing ensures that the random set satisfies Condition 5.1(a).

Proof. Due to stationarity, it is enough to consider $\mathcal{B}_{-\infty, 0}$ and $\mathcal{B}_{0, \infty}$. By the strong Markov property, the conditional distribution of the future $C \cap[0, \infty)$ of the random set $C$, given the past $\left.\xi\right|_{(-\infty, 0]}$ of the process, is the mixture of its conditional distributions given $(\tau, \xi(\tau))$, over the conditional distribution of $(\tau, \xi(\tau))$ given $\xi(0)$. Therefore $\xi(0)$ influences the conditional distribution but not its equivalence class.

The second condition of smearing. For every $t>0$, measures $T_{t}^{*} \delta_{a}$ (for all $a \in K)$ are (pairwise) equivalent.

The first condition of smearing constrains $\nu$ and $K_{0}$, while the second condition of smearing constrains only $\nu$, or rather, the semigroup $\left(T_{t}\right)$. Nevertheless:

6.3 Lemma. The second condition of smearing implies the first condition of smearing.

Proof. Assume the contrary: there exists a Borel set $B \subset[0, \infty) \times K_{0}$ such that the conditional probability $\mathbb{P}((\tau, \xi(\tau)) \in B \mid \xi(0)=a)$ vanishes for some but not all $a \in K \backslash K_{0}$. We may assume that $B \subset(0, \infty) \times K_{0}$ and moreover, $B \subset[t, \infty) \times K_{0}$ for some $t>0$. By the Markov property,

$$
\mathbb{P}((\tau, \xi(\tau)) \in B \mid \xi(0)=a)=\int_{K} \mathbb{P}((\tau, \xi(\tau)) \in B \mid \xi(t)=b) d\left(T_{t}^{*} \delta_{a}\right)(b) .
$$

The integral vanishes for some but not all $a$, in contradiction to equivalence of measures $T_{t}^{*} \delta_{a}$.

Specifically, we take a diffusion process $\xi(\cdot)$ on the two-dimensional torus $K=\mathbb{R}^{2} / \mathbb{Z}^{2}=\{(x, y): 0 \leq x \leq 1,0 \leq y \leq 1\}$ with the identification: $x=0$ to $x=1$, and $y=0$ to $y=1$. The process is governed by the differential operator

$$
L=\frac{1}{2} \frac{\partial^{2}}{\partial y^{2}}+f(y) \frac{\partial}{\partial x}+\frac{1}{2} g^{2}(y) \frac{\partial^{2}}{\partial x^{2}},
$$

where $f$ and $g$ are $C^{\infty}$ functions on the circle $\mathbb{R} / \mathbb{Z}$ such that

$$
\begin{gathered}
f(y)>0 \text { and } f^{\prime}(y)>0 \text { for } 0 \leq y \leq \frac{3}{4} \\
g(y)=0 \text { for } 0 \leq y \leq \frac{3}{4} ; \quad g(y)>0 \text { for } \frac{3}{4}<y<1 .
\end{gathered}
$$




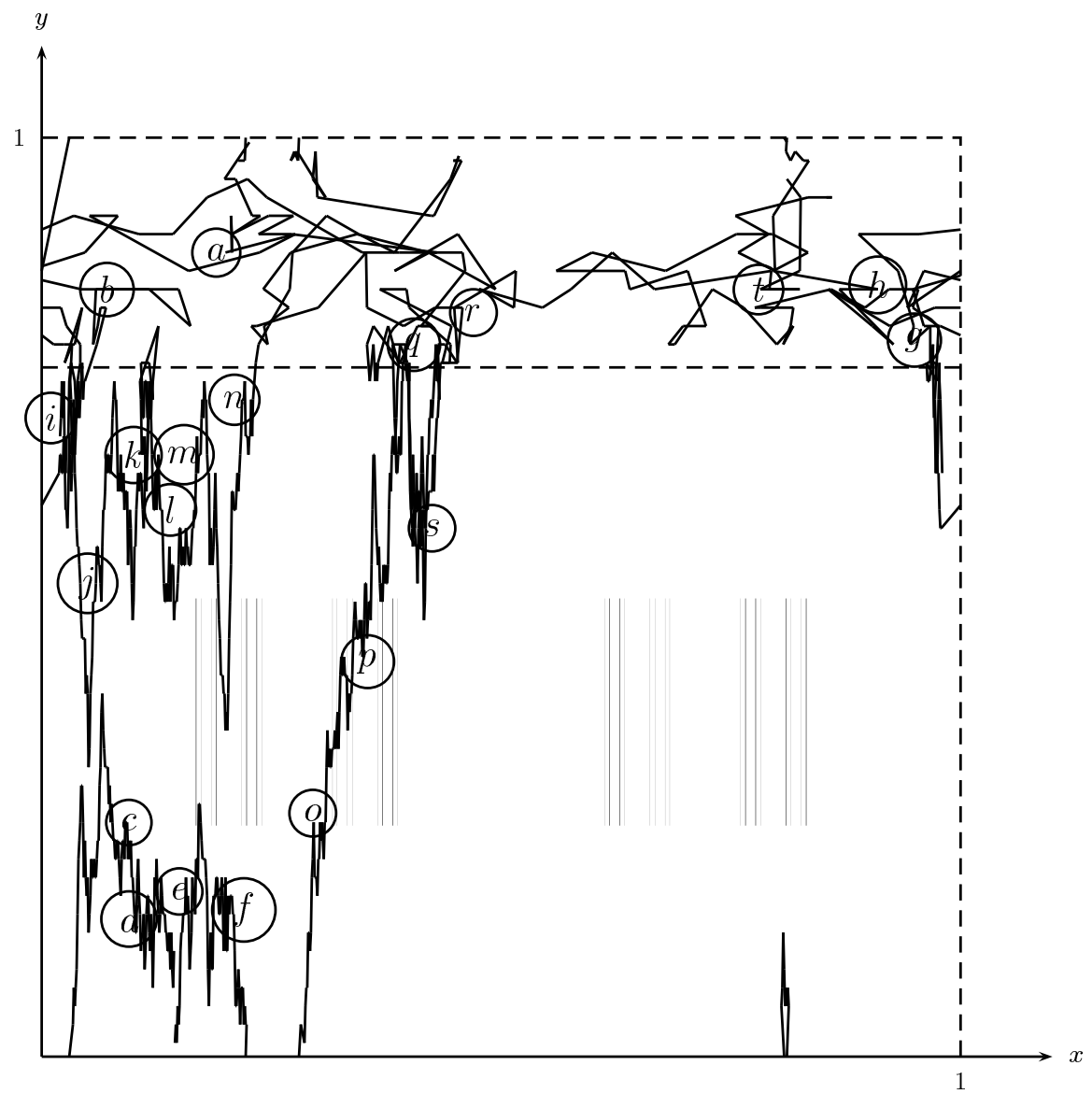

Figure 1: A sample path of the diffusion process on the torus. Time marks are shown by circled letters $a, b, \ldots, s, t$. The set $K_{0}$ is shown in gray.

In terms of coordinates, $\xi(t)=(x(t), y(t))$, we see that $y(\cdot)$ is just the standard Brownian motion (on the circle), and the behavior of $x(\cdot)$ depends on $y(\cdot)$ dramatically:

$$
x^{\prime}(t)=f(y(t)) \quad \text { when } 0<y(t)<\frac{3}{4} .
$$

Here, $x(\cdot)$ is continuously differentiable and monotone (on the circle); in contrast, when $\frac{3}{4}<y(t)<1, x(\cdot)$ is as irregular as the Brownian motion. See Fig. 1.

The subset $K_{0} \subset K$ is

$$
K_{0}=K_{1} \times\left[\frac{1}{4}, \frac{1}{2}\right]
$$

where $K_{1}$ is a perfect subset of $(0,1)$. 
The operator $L$ is not elliptic, since $g^{2}(\cdot)$ is not strictly positive. The degeneration impedes smearing, but gives us much better control over the random set. Indeed, the process $\xi(t)=(x(t), y(t))$ can hit $K_{0}$ only when $y(t) \in\left[\frac{1}{4}, \frac{1}{2}\right]$; here, the random set is determined by $x(t)$ only, and $x(t)$ is an increasing, continuously differentiable function. Thus, the random set is rather similar to the given set $K_{1}$.

On the other hand, it is essential that $f$ is not constant (on the relevant interval). Otherwise, if, say, $f(\cdot)=1$, the (local) metric structure of the set $K_{1}$ is reproduced by the random set exactly, with no random deformation, which is inconsistent with 5.1(a).

6.6 Lemma. The semigroup generated by $L$ satisfies the second condition of smearing.

Proof. Let $B$ be a Borel subset of the torus. We consider the function

$$
u(t, a)=\left(T_{t}^{*} \delta_{a}\right)(B) .
$$

It is enough to prove for each $t>0$, that either $u(t, a)=0$ for all $a \in K$, or $u(t, a)>0$ for all $a \in K$.

Assume for a while that $u$ is a smooth function, of class $C^{2}$; then $u$ is a classical solution of the partial differential equation $\left(\frac{\partial}{\partial t}-L\right) u=0$. It is a second order PDE with nonnegative characteristic form, and we may use the strong maximum principle based on elliptic connectivity, see [OR, Sect. 3.1]. Circles $\{(x, y, t): y \in \mathbb{R} / \mathbb{Z}\}$, parametrized by $x \in \mathbb{R} / \mathbb{Z}$ and $t>0$, are lines of ellipticity for the PDE $\left(\frac{\partial}{\partial t}-L\right) u=0$ (see [OR, p. 208] for the definition), due to the term $\partial^{2} / 2 \partial y^{2}$ of $L$. Also, circles $\{(x, y, t): x \in \mathbb{R} / \mathbb{Z}\}$ for $y \in\left(\frac{3}{4}, 1\right)$ and $t>0$ are lines of ellipticity, due to the term $g^{2}(y) \partial^{2} / 2 \partial x^{2}$. Thus, for each $t>0$, the torus $K \times\{t\}=\{(x, y, t): x, y \in \mathbb{R} / \mathbb{Z}\}$ is a set of elliptic connectivity (see [OR, p. 209]). By [OR, Th. 3.1.2], on such a set either $u=0$ everywhere, or $u>0$ everywhere.

It remains to prove that $u$ is of class $C^{2}$. (Now it is essential that $g(\cdot) \neq$ const.) The function $u$ is a weak solution of the $\operatorname{PDE}\left(\frac{\partial}{\partial t}-L\right) u=0$; it is enough to check hypoellipticity of the operator $\frac{\partial}{\partial t}-L$. According to the wellknown sufficient condition of Hörmander (see [OR, Th. 2.5.2]), it is enough to check that the system

$$
X_{1}=\frac{\partial}{\partial y}, \quad X_{2}=g(y) \frac{\partial}{\partial x}, \quad X_{0}=f(y) \frac{\partial}{\partial x}+\frac{\partial}{\partial t}
$$

of first order differential operators (these represent $L$ as $\frac{1}{2} X_{1}^{2}+\frac{1}{2} X_{2}^{2}+X_{0}$ ) has the full rank, equal to the dimension 3 of $K \times(0, \infty)$; see $\mathrm{OR}$, p. 167]. 
In the domain $\frac{3}{4}<y<1$ the full rank is evident, since vectors

$$
\left(\begin{array}{l}
0 \\
1 \\
0
\end{array}\right), \quad\left(\begin{array}{c}
g(y) \\
0 \\
0
\end{array}\right), \quad\left(\begin{array}{c}
f(y) \\
0 \\
1
\end{array}\right)
$$

are linearly independent. In the other domain, $0 \leq y \leq \frac{3}{4}, X_{2}$ vanishes, and we have only two operators. However, their commutator

$$
\left[X_{1}, X_{3}\right]=\frac{\partial}{\partial y}\left(f(y) \frac{\partial}{\partial x}+\frac{\partial}{\partial t}\right)-\left(f(y) \frac{\partial}{\partial x}+\frac{\partial}{\partial t}\right) \frac{\partial}{\partial y}=f^{\prime}(y) \frac{\partial}{\partial x}
$$

gives us the needed third vector.

We have $L \mathbf{1}=0$, but also $L^{*} \mathbf{1}=0$ ( since $L^{*}$ differs from $L$ only in the sign of $f$ ), which means that the Lebesgue measure $\nu_{0}$ on the torus $K$ is invariant under $\left(T_{t}^{*}\right)$.⿴囗十丁 Choosing once and for all functions $f$ and $g$, we hold fixed the (distribution $\nu$ of the) diffusion process $\xi(\cdot)$. The only parameter of our random set is the perfect set $K_{1} \subset(0,1)$ of Lebesgue measure 0 . Every such $K_{1}$ gives us a measure $\mu$ on $\mathcal{C}$, and its equivalence class $\mathcal{M}$ is decomposable; indeed, it satisfies Conditions 5.1 (a,b).

Recall that $K_{0}=K_{1} \times\left[\frac{1}{4}, \frac{1}{2}\right]$ is the union of vertical segments. The set $K_{1} \times\left\{\frac{1}{4}, \frac{1}{2}\right\}$ of their endpoints is polar, that is, never visited by the process $\xi$, under appropriate conditions on $K_{1}$, as will be shown by constructing a 'barrier function'.

6.7 Lemma. There exists a function $h: K \backslash\left\{\left(\frac{1}{2}, \frac{1}{2}\right)\right\} \rightarrow[0, \infty)$ such that

(a) $h$ is twice continuously differentiable on $K \backslash\left\{\left(\frac{1}{2}, \frac{1}{2}\right)\right\}$;

(b) $\sup _{(x, y) \in K \backslash\left\{\left(\frac{1}{2}, \frac{1}{2}\right)\right\}} \sqrt{\left|x-\frac{1}{2}\right|}|(L h)(x, y)|<\infty$;

(c) $\liminf _{x \rightarrow \frac{1}{2}-} \sqrt{\frac{1}{2}-x} h\left(x, \frac{1}{2}\right)>0$.

Proof. The function $h$ will be constructed on a (punctured) neighborhood of the point $\left(\frac{1}{2}, \frac{1}{2}\right)$, assuming that $f\left(\frac{1}{2}\right)=1$ (just to simplify calculations; the general case follows by rescaling $x$ or $y$ ). For convenience, we turn from $x$ and $y$ to new coordinates $z=y-\frac{1}{2}, t=\frac{1}{2}-x ;$ now,

$$
L=\frac{1}{2} \frac{\partial^{2}}{\partial z^{2}}-\left(1+\alpha z+O\left(z^{2}\right)\right) \frac{\partial}{\partial t}
$$

\footnotetext{
${ }^{15}$ Using hypoellipticity and the strong maximum principle, it is easy to see that the invariant measure is unique. However, we do not need it.

${ }^{16}$ The variable $t$, local to the proof, has nothing in common with, say, $t$ of $\left(\frac{\partial}{\partial t}-L\right)$.
} 
where $\alpha=f^{\prime}(1 / 2)$. We define

$$
h(z, t)=\frac{1}{\sqrt{t}} \exp \left(-\frac{z^{2}}{2 t}+a \frac{z^{3}}{t}+a z\right) \text { for } t>0
$$

and $h(z, t)=0$ for $t \leq 0$; parameter $a \in \mathbb{R}$ will be chosen later.

Condition (c) becomes

$$
\liminf _{x \rightarrow 0+} \sqrt{t} h(0, t)>0,
$$

which clearly holds. Condition (b) becomes

$$
L h(z, t)=O(1 / \sqrt{t}) \text { for } t>0 ;
$$

this one remains to be checked (for some $a$ ).

We have

$$
\frac{\partial^{2}}{\partial z^{2}} h(z, t)=\frac{1}{\sqrt{t}} \exp (\ldots) \cdot\left(\left(-\frac{z}{t}+3 a \frac{z^{2}}{t}+a\right)^{2}+\left(-\frac{1}{t}+6 a \frac{z}{t}\right)\right)
$$

Substituting $z=r \sqrt{t}$,

$$
\begin{array}{r}
\frac{\partial^{2}}{\partial z^{2}} h(z, t)=\frac{1}{\sqrt{t}} \exp \left(-\frac{r^{2}}{2}+a r^{3} \sqrt{t}+a r \sqrt{t}\right) \cdot\left(\left(-\frac{r}{\sqrt{t}}+3 a r^{2}+a\right)^{2}-\frac{1}{t}+6 a \frac{r}{\sqrt{t}}\right) \\
=\frac{1}{\sqrt{t}} \exp (\ldots) \cdot\left(\frac{r^{2}}{t}-6 a \frac{r^{3}}{\sqrt{t}}-2 a \frac{r}{\sqrt{t}}+O\left(r^{4}+1\right)-\frac{1}{t}+6 a \frac{r}{\sqrt{t}}\right)= \\
=\frac{1}{\sqrt{t}} \exp (\ldots) \cdot\left(\frac{r^{2}-1}{t}+\frac{4 a r-6 a r^{3}}{\sqrt{t}}+O\left(r^{4}+1\right)\right) .
\end{array}
$$

Similarly,

$$
\begin{aligned}
\frac{\partial}{\partial t} h(z, t)=\frac{1}{\sqrt{t}} \exp (\ldots) \cdot\left(-\frac{1}{2 t}+\right. & \left.\frac{z^{2}}{2 t^{2}}-a \frac{z^{3}}{t^{2}}\right)= \\
& =\frac{1}{2 \sqrt{t}} \exp (\ldots) \cdot\left(\frac{r^{2}-1}{t}-2 a \frac{r^{3}}{\sqrt{t}}\right) .
\end{aligned}
$$

Taking into account that $r \sqrt{t}=z=O(1)$ we get

$$
\begin{aligned}
& \operatorname{Lh}(z, t)=\frac{1}{2 \sqrt{t}} \exp (\ldots) \times \\
& \times\left(\frac{r^{2}-1}{t}+\frac{4 a r-6 a r^{3}}{\sqrt{t}}+O\left(r^{4}+1\right)-\left(1+\alpha r \sqrt{t}+O\left(r^{2} t\right)\right)\left(\frac{r^{2}-1}{t}-2 a \frac{r^{3}}{\sqrt{t}}\right)\right)= \\
& =\frac{1}{2 \sqrt{t}} \exp (\ldots) \cdot\left(\frac{4 a r-6 a r^{3}+2 a r^{3}-\alpha r\left(r^{2}-1\right)}{\left.\sqrt{t}+O\left(r^{4}+1\right)\right)=}\right. \\
& \quad=\frac{1}{2 \sqrt{t}} \exp (\ldots) \cdot\left(-\frac{(4 a+\alpha) r\left(r^{2}-1\right)}{\sqrt{t}}+O\left(r^{4}+1\right)\right) .
\end{aligned}
$$


We choose the parameter

$$
a=-\frac{\alpha}{4}
$$

and get

$$
\operatorname{Lh}(z, t)=\frac{1}{2 \sqrt{t}} \exp \left(-\frac{r^{2}}{2}+a r^{3} \sqrt{t}+a r \sqrt{t}\right) \cdot O\left(r^{4}+1\right) .
$$

For $|z|$ small enough (namely, $\left.|z| \leq \frac{1}{4|a|}\right)$,

$$
|L h(z, t)| \leq \frac{1}{2 \sqrt{t}} \exp \left(-\frac{r^{2}}{4}\right) \cdot O\left(r^{4}+1\right)=O\left(\frac{1}{\sqrt{t}}\right) \text {. }
$$

From now on we restrict ourselves to sets $K_{1}$ such that

$$
\Lambda_{1 / 2}\left(K_{1}\right)=0
$$

where $\Lambda_{1 / 2}$ is the Hausdorff measure of dimension $1 / 2$. The condition is satisfied by all sets $K_{1}$ of Hausdorff dimension $<1 / 2$, and some sets of Hausdorff dimension $=1 / 2$.

6.9 Lemma. Almost surely, the process $\xi(\cdot)$ does not hit the set $K_{1} \times\left\{\frac{1}{4}, \frac{1}{2}\right\}$.

Proof. By (6.8), for every $\varepsilon$, the set $K_{1}$ can be covered by a collection of intervals $\left[x_{k}, x_{k}+\delta_{k}\right]$ such that $\sum_{k} \sqrt{\delta_{k}} \leq \varepsilon$. Consider such a function on $K$ :

$$
u_{\varepsilon}(x, y)=\sum_{k} \sqrt{\delta_{k}} h\left(x-x_{k}-2 \delta_{k}+\frac{1}{2}, y\right) \text {, }
$$

where $h$ is given by Lemma 6.4. Condition 6.7(b) implies

$$
\int_{0}^{1} \sup _{y}|\operatorname{Lh}(x, y)| d x \leq \int \frac{d x}{\sqrt{\left|x-\frac{1}{2}\right|}}=C<\infty
$$

and therefore

$$
\int_{0}^{1} \sup _{y}\left|L u_{\varepsilon}(x, y)\right| d x \leq C \sum_{k} \sqrt{\delta_{k}} \leq C \varepsilon .
$$

Condition 6.7(c) gives us $c>0$ such that

$$
u_{\varepsilon}\left(x, \frac{1}{2}\right)>c \text { for each } x \in K_{1} \text {. }
$$


Also,

$$
\left|u_{\varepsilon}(x, y)\right| \leq M_{y} \sum_{k} \sqrt{\delta_{k}} \leq M_{y} \varepsilon
$$

where $M_{y}=\sup _{x} h(x, y)<\infty$ for $y \neq \frac{1}{2}$. Note that $c, C, M_{y}$ do not depend on $\varepsilon$. We do so for each $\varepsilon$ of a sequence $\varepsilon_{1}, \varepsilon_{2}, \ldots$ such that $\sum \varepsilon_{n}<\infty$ and consider

$$
u(x, y)=\sum_{n} u_{\varepsilon_{n}}(x, y)
$$

Then

$$
\begin{gathered}
\int_{0}^{1} \sup _{y}|L u(x, y)| d x \leq C \sum_{n} \varepsilon_{n}<\infty, \\
u\left(x, \frac{1}{2}\right)=\infty \quad \text { for each } x \in K_{1}, \\
|u(x, y)| \leq M_{y} \sum_{n} \varepsilon_{n}<\infty \quad \text { for each } y \neq \frac{1}{2} .
\end{gathered}
$$

The random process $u(\xi(\cdot))$ is a semimartingale, whose drift is $(L u)(\xi(\cdot))$. When inside the region $0<y<\frac{3}{4}$, the drift is locally integrable (in time) due to $(6.10)$. Assume that a sample path $\xi(\cdot)$ hits $K_{1} \times\left\{\frac{1}{2}\right\}$ at some time $t$ the first time. Then the martingale part of the semimartingale at $t-$ is bounded from below but unbounded from above (due to (6.11)), which can happen only with probability 0 . The same holds for $K_{1} \times\left\{\frac{1}{4}\right\}$.

Given a sample path $\xi(\cdot)$ of our diffusion process, we may consider two closed sets on the time axis; one of them is our random set $C=\{t: \xi(t) \in$ $\left.K_{1} \times\left[\frac{1}{4}, \frac{1}{2}\right]\right\}$, the other is $D=\left\{t: \xi(t) \in \mathbb{R} / \mathbb{Z} \times\left\{\frac{1}{4}, \frac{1}{2}\right\}\right\}$. In terms of coordinates $x(t), y(t)$ of the point $\xi(t), D=\left\{t: y(t)=\frac{1}{4}\right\} \cup\left\{t: y(t)=\frac{1}{2}\right\}$. By Lemma 6.5, $C \cap D=\emptyset$. Both sets are unbounded. We get two alternating sequences, $\cdots<s_{-1}<t_{-1}<s_{0}<t_{0}<s_{1}<t_{1}<s_{2}<\ldots$ such that $s_{k} \in C, t_{k} \in D,\left(s_{k}, t_{k}\right) \cap D=\emptyset,\left(t_{k}, s_{k+1}\right) \cap C=\emptyset$ for all $k \in \mathbb{Z}$. Thus, $C \subset \cup_{k}\left[s_{k}, t_{k}\right]$, and for all $t \in \cup_{k}\left[s_{k}, t_{k}\right]$ we have $\frac{1}{4} \leq y(t) \leq \frac{1}{2}$; therefore $x^{\prime}(t)=f(y(t))$ - recall (6.5). A portion $C \cap\left[s_{k}, t_{k}\right]$ of the set $C$, equal to $\left\{t \in\left[s_{k}, t_{k}\right]: x(t) \in K_{1}\right\}$, results from the corresponding portion of $K_{1}$ by a smooth (of class $C^{1}$ ) monotone transformation $\left.x(\cdot)\right|_{\left[s_{k}, t_{k}\right]}$. Of course, "the corresponding portion of $K_{1}$ ' should be understood cyclically; $K_{1} \subset \mathbb{R} / \mathbb{Z}$, and $x(\cdot)$ can make several revolutions during $\left[s_{k}, t_{k}\right]$. Note also that $f\left(\frac{1}{4}\right) \leq$ $x^{\prime}(t) \leq f\left(\frac{1}{2}\right)$ for $t \in \cup_{k}\left[s_{k}, t_{k}\right]$. The numbers $f\left(\frac{1}{4}\right), f\left(\frac{1}{2}\right)$ are arbitrary, except for the constraint $0<f\left(\frac{1}{4}\right)<f\left(\frac{1}{2}\right)$. For every $\varepsilon>0$ the function $f$ can be chosen so that $f\left(\frac{1}{4}\right)=1$ and $f\left(\frac{1}{2}\right)=1+\varepsilon$, ensuring $1 \leq x^{\prime}(t) \leq 1+\varepsilon$ for $t \in \cup_{k}\left[s_{k}, t_{k}\right]$. 
The Hausdorff dimension of $C \cap\left[s_{k}, t_{k}\right]$ is evidently equal to the Hausdorff dimension of the corresponding portion of $K_{1}$. If the local Hausdorff dimension of $K_{1}$ is equal to a given $\alpha$ at every point of $K_{1}$, then the same holds for $C$. Such a $K_{1}$ exists for each $\alpha \in\left(0, \frac{1}{2}\right)$; it leads to a decomposable equivalence class of measures on $\mathcal{C}$ (Lemmas 6.2, 6.3, 6.6), a $\mathrm{CP} \mathrm{MC}$ (Sect. 5), and a CP HS (Sect. 3) of type $I I_{0}$ (Lemma 5.8). They all are invariant under time shifts; thus, the CP HS is in fact an HCP HS and leads to a product system (Theorem 1.9) of type $I I_{0}$ (that is, having one-dimensional spaces $D(t)$ of decomposable vectors, see [A, Remark 3.3.3]). These product systems for different $\alpha$ are non-isomorphic (Corollary 5.9 and Lemma 1.10), which proves the following result.

6.13 Theorem. There is a continuum of mutually non-isomorphic product systems of type $I I_{0}$.

It answers in the affirmative two questions of Arveson [A96, p. 12]: (1) Are there uncountably many non-isomorphic product systems? (2) (see also [A99, p. 167]) Is there a product system of type $I I_{0}$ ?

\section{Time reversal}

Recalling Sect. 1, a history $\left(\left(e^{i t X}\right)_{t \in \mathbb{R}}, \mathcal{B}\left(H_{-}\right) \otimes \mathbf{1}\right)$ leads to an $E_{0}$-semigroup $\alpha^{+}$and its product system. The time-reversed history $\left(\left(e^{-i t X}\right)_{t \in \mathbb{R}}, \mathbf{1} \otimes \mathcal{B}\left(H_{+}\right)\right)$ leads to another $E_{0}$-semigroup $\alpha^{-}$and another product system. These two product systems are called opposite (to each other); see [A], Sect. 3.5, 3.6].

For any CP HS $\left(\left(H_{s, t}\right)_{-\infty \leq s<t \leq \infty},\left(W_{r, s, t}\right)_{-\infty \leq r<s<t \leq \infty}\right)$, its time-reversed CP HS is, by definition, $\left(\left(\tilde{H}_{s, t}\right)_{-\infty \leq s<t \leq \infty},\left(\tilde{W}_{r, s, t}\right)_{-\infty \leq r<s<t \leq \infty}\right)$, where $\tilde{H}_{s, t}=$ $H_{-t,-s}$ and $\tilde{W}_{r, s, t}(x \otimes y)=W_{-t,-s,-r}(y \otimes x)$ for $x \in \tilde{H}_{r, s}, y \in \tilde{H}_{s, t}$. For an HCP HS, we add $\tilde{\theta}_{s, t}^{u}=\theta_{-t,-s}^{-u}$.

Each tail trivial HCP HS leads to a history (Corollary 1.8). Each HCP HS leads to a product system (Theorem 1.9). The time-reversed HCP HS leads to the time-reversed history (under tail triviality), and the opposite product system (irrespective of tail triviality).

For a $\mathrm{CP} \operatorname{MC}\left((X, \mathcal{B}, \mathcal{M}),\left(\mathcal{F}_{s, t}\right)_{-\infty \leq s<t \leq \infty}\right)$ (recall Definition 3.10), its time-reversed $\mathrm{CP} \mathrm{MC}$ is, by definition, $\left((X, \mathcal{B}, \mathcal{M}),\left(\mathcal{F}_{-t,-s}\right)_{-\infty \leq s<t \leq \infty}\right)$. It leads to the time-reversed CP HS. The same for the homogeneous case (HCP $\mathrm{MC}$ and HCP HS), and for CPs PS (homogeneous or not).

For a decomposable equivalence class of measures on $(\mathcal{C}, \mathcal{B})$ (recall Definition 5.1), its time-reversed equivalence class of measures on $(\mathcal{C}, \mathcal{B})$ is, by definition, its image under the map $\mathcal{C} \ni C \mapsto\{t:(-t) \in C\} \in \mathcal{C}$. It leads to the time-reversed CP HS. (Homogeneity is respected, too.) 
For the setup of the beginning of Sect. 6 (recall $\left.K,\left(T_{t}\right), \nu, \nu_{0}\right)$, the timereversed random process is described by the image $\tilde{\nu}$ of $\nu$ under the map $C(\mathbb{R}, K) \ni \xi \mapsto \tilde{\xi} \in C(\mathbb{R}, K)$ given by $\tilde{\xi}(t)=\xi(-t)$. It corresponds to the same measure $\nu_{0}$ on $K$, but a different semigroup $\left(\tilde{T}_{t}\right)$,

$$
\int_{K}\left(\tilde{T}_{t} f\right) g d \nu_{0}=\int_{K} f\left(T_{t} g\right) d \nu_{0}
$$

for all $f, g \in C(K)$. Indeed, such $\left(\tilde{T}_{t}\right)$ satisfies a time-reversed version of (6.1),

$$
\begin{aligned}
\int_{C(\mathbb{R}, K)} f_{1}\left(\xi\left(t_{1}\right)\right) \ldots & f_{n}\left(\xi\left(t_{n}\right)\right) d \nu(\xi)= \\
& =\int_{C(\mathbb{R}, K)}\left(\tilde{T}_{t_{2}-t_{1}} f_{1}\right)\left(\xi\left(t_{2}\right)\right) f_{2}\left(\xi\left(t_{2}\right)\right) \ldots f_{n}\left(\xi\left(t_{n}\right)\right) d \nu(\xi) .
\end{aligned}
$$

(Use (6.1) for reducing $n$ by 1 until $n=2$.)

For the semigroup $\left(T_{t}\right)$ generated by $L$ of (6.4), the time-reversed semigroup $\left(\tilde{T}_{t}\right)$ is generated by the operator

$$
L^{*}=\frac{1}{2} \frac{\partial^{2}}{\partial y^{2}}-f(y) \frac{\partial}{\partial x}+\frac{1}{2} g^{2}(y) \frac{\partial^{2}}{\partial x^{2}},
$$

which shows that the time-reversed process $\tilde{\xi}(\cdot)$ may be identified (in distribution) with the process $(1-x(t), y(t))$, where $(x(t), y(t))=\xi(t)$; here, time reversal appears to be equivalent to $x$ reversal.

If the $x$ reversal leaves $K_{1}$ invariant, that is, $x \in K_{1}$ if and only if (1$x) \in K_{1}$ for all $x \in(0,1)$ (which is evidently compatible with any Hausdorff dimension), then our random set is symmetric (in distribution), that is, the corresponding decomposable equivalence class of measures is isomorphic to its time reversal, which gives us a sharpening of Theorem 6.13.

7.1 Theorem. There is a continuum of mutually nonisomorphic symmetrip product systems of type $I I_{0}$.

In order to find asymmetric product systems, we need another invariant of a perfect set; indeed, Hausdorff dimensions of $K_{1}$ and $\tilde{K}_{1}=\left\{x:(1-x) \in K_{1}\right\}$ are evidently equal.

Let us restrict ourselves to perfect sets $K_{1}$ such that each connected component of the complement $(\mathbb{R} / \mathbb{Z}) \backslash K_{1}$ is an interval of length of the

\footnotetext{
${ }^{17}$ Each isomorphic to its opposite, in other words, anti-isomorphic to itself.
} 
form, say, $10^{-n}, n=1,2, \ldots$ On the other hand, we choose $f$ such that $f\left(\frac{1}{4}\right)=1$ and $f\left(\frac{1}{2}\right) \leq 2$. The (random) transformation, relating a portion of the random set $C$ to a portion of $K_{1}$, cannot change a length too much (as explained before Theorem 6.13). For each component of $\mathbb{R} \backslash C$, the corresponding exponent $n$ is determined uniquely, unless the component is situated between two portions. Thus, the combinatorial structure of $K_{1}$ is inherited by $C$, though a (locally) finite number of high hierarchical levels are spoiled by switching between portions. By the combinatorial structure I mean data like the following: between a pair of nearest intervals of length $10^{-20}$ there are three intervals of length $10^{-21}$; before the first of these three we see two intervals of length $10^{-22}$, while between the first and second (of the three) we see only one interval of length $10^{-22} \ldots$, etc. An infinite array of data may be encoded in the combinatorial structure of $K_{1}$, and most of the data are preserved in $C$. It gives us an alternative way to Theorem 6.13, unrelated to the Hausdorff dimension. More important, it gives us a lot of asymmetric random sets; indeed, the combinatorial structure need not be symmetric at all. The idea may be implemented as follows.

Let $a=\left(a_{k}\right)_{k=1}^{\infty}, b=\left(b_{k}\right)_{k=1}^{\infty}$ be two infinite sequences of numbers 0 and 1. Introduce the set $M_{a, b}$ of all numbers of the form $\sum_{k=1}^{n} c_{k} 3^{-k}$, where $n \in$ $\{1,2, \ldots\}$, each $c_{k}$ is either -1 or +1 , and the combination $c_{2 k}=c_{2 k+1}=-1$ is forbidden for each $k$ such that $a_{k}=0$; similarly, the combination $c_{2 k}=$ $c_{2 k+1}=+1$ is forbidden whenever $b_{k}=0$. For any $x=\sum_{k=1}^{n} c_{k} 3^{-k} \in M_{a, b}$ we define $p(x)=10^{-n}$, and consider the closure $K_{a, b}$ of the set

$$
\left\{\sum_{x \in M_{a, b} \cap(-\infty, y)} p(x): y \in \mathbb{R}\right\} .
$$

7.2 Lemma. (a) For any sequences $a, b$ the set $K_{a, b}$ is a perfect set of Lebesgue measure zero.

(b) Let $x \in K_{a, b}, \varepsilon>0$, and $f:(x-\varepsilon, x+\varepsilon) \rightarrow \mathbb{R}$ be an increasing function such that

$$
\frac{1}{5} \leq \frac{f(t)-f(s)}{t-s} \leq 5 \quad \text { whenever } x-\varepsilon \leq s<t \leq x+\varepsilon .
$$

Assume that $a^{\prime}, b^{\prime}$ is another pair of sequences such that for every $t \in(x-$ $\varepsilon, x+\varepsilon)$,

$$
t \in K_{a, b} \text { if and only if } f(t) \in K_{a^{\prime}, b^{\prime}} .
$$

Then $a_{k}=a_{k}^{\prime}$ and $b_{k}=b_{k}^{\prime}$ for all $k$ large enough.

(c) Assume the same as in (b), but let $f$ be a decreasing function, $\frac{1}{5} \leq$ $\frac{f(s)-f(t)}{t-s} \leq 5$. Then $a_{k}=b_{k}^{\prime}$ and $b_{k}=a_{k}^{\prime}$ for all $k$ large enough.

\footnotetext{
${ }^{18}$ Or, less demanding, of length that belongs to $\cup_{n}\left[10^{-n}, 2 \cdot 10^{-n}\right]$.
} 
The proof is left to the reader. The conclusion follows.

7.3 Theorem. There is a continuum of mutually non-isomorphic asymmetric product systems of type $I I_{0}$.

It answers in the negative a question of Arveson [A89, p. 6], namely, whether every product system is symmetric, or not.

\section{FHS space: logarithm of a Hilbert space}

... we have not yet achieved a satisfactory understanding of the existence of "logarithms" for product systems.

W. Arveson [A丹, Sect. 1.6]

The classical understanding of the well-known Fock [-Cook] exponentiation was widely believed to be satisfactory, but is now changing dramatically. See [A, Sect. 3.3.1] for a concise summary of the classical understanding; a Hilbert space $e^{H}$ arises from a given Hilbert space $H$, and $e^{H_{1} \oplus H_{2}}=e^{H_{1}} \otimes e^{H_{2}}$ (up to a canonical isomorphism), and the unitary group of $H$ acts on $e^{H}$ unitarily, by $U \mapsto \Gamma(U)$. Close relations to Gaussian random variables and Weil (canonical) commutation relations are well-known, see [Ja, Chap. IV and XIII].

Let us start with a finite-dimensional Hilbert space $H$ over $\mathbb{R}$ (that is, Euclidean space). It carries the $n$-dimensional Lebesgue measure $(n=\operatorname{dim} H)$, denoted by $\operatorname{mes}_{H}$. We may simply define

$$
e^{H}=L_{2}\left(\left(H, \operatorname{mes}_{H}\right), \mathbb{R}\right),
$$

the space of square integrable real-valued functions on $H$; its complexification is $e_{\mathbb{C}}^{H}=L_{2}\left(\left(H, \operatorname{mes}_{H}\right), \mathbb{C}\right)$. Why denote the space by $e^{H}$ ? Since $e^{H_{1} \oplus H_{2}}=$ $e^{H_{1}} \otimes e^{H_{2}}$ (just because mes $_{H_{1} \oplus H_{2}}=$ mes $_{H_{1}} \times \operatorname{mes}_{H_{2}}$ ). The action $U \mapsto \Gamma(U)$ is evident: $\Gamma(U) f(x)=f\left(U^{-1} x\right)$. Also, the additive group of $H$ acts on $e^{H}$ (and $e_{\mathbb{C}}^{H}$ ) by shifts, $\left(V_{x} f\right)(y)=f(x+y)$. Note that $\Gamma(U) V_{x}=V_{U x} \Gamma(U)$, which means that $\Gamma$ and $V$ together form a representation of the group of motions of $H . \mathbb{T}$ On the complex space $e_{\mathbb{C}}^{H}$ there is another action of the additive group of $H$, by such multiplications: $\left(U_{x} f\right)(y)=e^{i\langle x, y\rangle} f(y)$; these unitary operators satisfy the Weil relations, $U_{x} V_{y}=e^{-i\langle x, y\rangle} V_{y} U_{x}$. There is $f_{0} \in e_{\mathbb{C}}^{H}$ such that $\left\langle V_{x} f_{0}, f_{0}\right\rangle=\exp \left(-\frac{1}{8}\|x\|^{2}\right)$ and $\left\langle U_{x} f_{0}, f_{0}\right\rangle=\exp \left(-\frac{1}{2}\|x\|^{2}\right)$

\footnotetext{
${ }^{19}$ Given that $\Gamma(U) f(x)$ is $f\left(U^{-1} x\right)$ rather than $f(U x)$, it would be more consistent to define $V_{x} f(y)$ as $f(y-x)$ rather than $f(y+x)$. However, $f(y+x)$ conforms to the tradition of Weil relations.
} 
for all $x$, ${ }^{20}$ namely, $f_{0}(x)=(2 \pi)^{-n / 4} \exp \left(-\frac{1}{4}\|x\|^{2}\right)$ where $n=\operatorname{dim} H$; and it is unique up to a phase coefficient. The measure $\gamma_{H}=\left|f_{0}\right|^{2} \cdot$ mes $_{H}$, that is, $(2 \pi)^{-n / 2} \exp \left(-\frac{1}{2}\|x\|^{2}\right) d x$, is the standard Gaussian measure on $H$. Note that $\gamma_{H_{1} \oplus H_{2}}=\gamma_{H_{1}} \times \gamma_{H_{2}}$. Thus, we may replace (8.1) with

$$
e^{H}=L_{2}\left(\left(H, \gamma_{H}\right), \mathbb{R}\right)
$$

and get $e^{H_{1} \oplus H_{2}}=e^{H_{1}} \otimes e^{H_{2}}$ again, as well as the unitary representation $\Gamma(U) f(x)=f\left(U^{-1} x\right)$. And no wonder; the two spaces, $L_{2}\left(\left(H, \operatorname{mes}_{H}\right), \mathbb{R}\right)$ and $L_{2}\left(\left(H, \gamma_{H}\right), \mathbb{R}\right)$ may be identified by the canonical unitary map, $L_{2}\left(\left(H, \gamma_{H}\right), \mathbb{R}\right) \ni f \mapsto f \cdot\left|f_{0}\right|^{2} \in L_{2}\left(\left(H, \operatorname{mes}_{H}\right), \mathbb{R}\right)$. Formulas (8.1), (8.2) may be united (recall Sect. 3):

$$
e^{H}=L_{2}\left(\left(H, \mathcal{M}_{H}\right), \mathbb{R}\right),
$$

where $\mathcal{M}_{H}$ is the equivalence class of measures on $H$ that contains mes ${ }_{H}$ and $\gamma_{H}$. The measure class $\left(H, \mathcal{M}_{H}\right)$ will be called the standard measure class of $H$.

Taking into account that $\operatorname{mes}_{H}$ and $\gamma_{H}$ fail in infinite dimension, we reformulate the framework. The first twist: we treat $\gamma_{H}$ as a measure on the dual space $H^{\prime}$ (the space of all linear maps $H \rightarrow \mathbb{R}$ ) rather than $H$. It is basically the same, due to the evident canonical correspondence between $H$ and $H^{\prime}, x \mapsto\langle\cdot, x\rangle$. The random element of $H^{\prime}$ is a random linear function on $H$. The second twist: we restrict the random function to a basis of $H$. Nothing is changed in finite dimension, since a linear functional is uniquely determined by its restriction to the basis. However, in infinite dimension we escape the problem of (dis)continuity of the functional. That is the idea; its implementation follows.

Let $H$ be an infinite-dimensional separable Hilbert space over $\mathbb{R}$, and $E$ an orthonormal basis of $H$ (treated as a countable subset of $H$ rather than a sequence). The space $\mathbb{R}^{E}$ of all functions $\xi: E \rightarrow \mathbb{R}$ is the product $\prod_{e \in E} \mathbb{R}$ of countably many copies of $\mathbb{R}$. We equip (each copy of) $\mathbb{R}$ with the standard Gaussian measure $\gamma^{1}$ (just $(2 \pi)^{-1 / 2} e^{-u^{2} / 2} d u$ ) and consider the probability space

$$
\left(\mathbb{R}, \gamma^{1}\right)^{E}=\prod_{e \in E}\left(\mathbb{R}, \gamma^{1}\right)=\left(\mathbb{R}^{E}, \gamma^{E}\right)
$$

Of course, it is isomorphic to the space of sequences $\left(\mathbb{R}, \gamma^{1}\right)^{\mathbb{N}}=\left(\mathbb{R}^{\mathbb{N}}, \gamma^{\mathbb{N}}\right)$, $\mathbb{N}=\{1,2, \ldots\}$. An evident isomorphism between $\left(\mathbb{R}^{E}, \gamma^{E}\right)$ and $\left(\mathbb{R}^{E^{\prime}}, \gamma^{E^{\prime}}\right)$ suggests itself for any two orthonormal bases $E, E^{\prime} \subset H$ (provided that

\footnotetext{
${ }^{20}$ Coefficients $\frac{1}{8}, \frac{1}{2}$ (rather than $\frac{1}{4}, \frac{1}{4}$ ) may seem strange, but conform to probabilistic tradition.
} 
the bases are enumerated). However, the evident isomorphism is irrelevant; what we need, is another, not so evident isomorphism between these spaces, constructed below.

Given an orthonormal basis $E$ and a vector $x \in H$, we may define $\xi_{x}$ for almost all $\xi \in \mathbb{R}^{E}$ by

$$
\xi_{x}=\sum_{e \in E}\langle x, e\rangle \xi_{e}
$$

the series (of orthogonal terms) converges in $L_{2}\left(\mathbb{R}^{E}, \gamma^{E}\right)$. ${ }^{2}$ Thus, we extend $\xi$ from $E$ to $E \cup\{x\} .{ }^{22}$ It is easy to see that $\xi_{x}$ is a normal random variable, $\xi_{x} \sim$ $N\left(0,\|x\|^{2}\right)$. Also, pairs $\left(\xi_{x}, \xi_{y}\right)$ have two-dimensional normal distributions, $\mathbb{E} \xi_{x} \xi_{y}=\langle x, y\rangle$.

Given the second orthonormal basis $E^{\prime} \subset H$, we extend $\xi$ from $E$ to $E \cup E^{\prime}$ and observe that the distribution of the family $\left(\xi_{e}\right)_{e \in E^{\prime}}$ is equal to $\gamma^{E^{\prime}}$. We get a (non-evident) isomorphism between the two probability spaces, $\left(\mathbb{R}^{E}, \gamma^{E}\right)$ and $\left(\mathbb{R}^{E^{\prime}}, \gamma^{E^{\prime}}\right)$.

Does it mean that we can glue together probability spaces $\left(\mathbb{R}^{E}, \gamma^{E}\right)$ for all bases $E$ ? On the level of individual points (of probability spaces), it does not. Indeed, each isomorphism is defined almost everywhere; the union of a continuum of negligible sets need not be negligible.23 However, on the level of equivalence classes (of measurable sets or measurable functions) the glueing is well-defined, and we get a single Hilbert space

$$
e^{H}=L_{2}\left(\mathbb{R}^{E}, \gamma^{E}\right) \text { for all } E,
$$

where $E$ runs over orthonormal bases of $H$. In particular, for every vector $x \in H$, the random variable $\xi_{x} \in e^{H}$ is well-defined, $\xi_{x} \sim N\left(0,\|x\|^{2}\right), \mathbb{E} \xi_{x} \xi_{y}=$ $\langle x, y\rangle$, and the joint distributions of such random variables are normal. We get the object well-known as 'the isonormal process on $H$ ' or 'a Gaussian Hilbert space indexed by $H$ ', see [Da, Def. 1.18]. A shorter description is given there, at the expense of considerable arbitrariness. The description proposed above is canonical; everything is uniquely determined by $H$ (not only up to isomorphism), as far as negligible sets are neglected.

Till now, it is just another description of the classical $e^{H}$ for a real Hilbert space $H$. Recall however $\left(H, \mathcal{M}_{H}\right)$ in (8.3). For the infinite dimension we may still rewrite (8.4) as

$$
e^{H}=L_{2}\left(\mathbb{R}^{E}, \mathcal{M}^{E}\right) \text { for all } E,
$$

\footnotetext{
${ }^{21}$ In fact, it converges almost everywhere (provided that the basis is enumerated), but we do not need it.

${ }^{22}$ Note that we do not try to extend $\xi$ to the whole $H$.

${ }^{23}$ In fact, here it appears to be co-negligible.
} 
where $\mathcal{M}^{E}$ is the equivalence class of measures that contains $\gamma^{E}$. Doing so, we have no reason to restrict ourselves to orthonormal bases and measure preserving maps!

For a finite-dimensional $H$, each basis $E$ (orthonormal or not) can be used. The joint distribution of random variables $\xi_{e}, e \in E$, is some non-degenerate normal distribution (Gaussian measure) in $\mathbb{R}^{n}$ (not just $\gamma^{n}$ ); anyway, it belongs to the standard measure class $\mathcal{M}_{H}$.

In infinite dimension we cannot use arbitrary bases of $H$. Even such a seemingly innocent case as $\left\langle e_{k}, e_{l}\right\rangle=0$ but, say, $\left\langle e_{k}, e_{k}\right\rangle=2$ (rather than 1 ), leads to a distribution non-equivalent to $\gamma^{\mathbb{N}}$. Still, some non-orthonormal bases can be used. In other words, we can use bases orthonormal w.r.t. some other norms on $H$. The relevant equivalence relation for norms was described in 1958 by J. Feldman, J. Hájek and I. Segal, see [A, Th. 3.3.7], А†, Th. 14.3.1]. The following definition is applicable both to real and complex spaces, however, all FHS spaces will be assumed real, unless otherwise stated.

8.5 Definition. (a) An FHS-equivalence operator between separable Hilbert spaces $H_{1}, H_{2}$ is a linear homeomorphism $L: H_{1} \rightarrow H_{2}$ such that $\mathbf{1}-L^{*} L$ is a Hilbert-Schmidt operator on $H_{1}$.

(b) FHS-equivalent norms on a linear space $E$ are norms $\|\cdot\|_{1},\|\cdot\|_{2}$ on $E$ such that $\left(E,\|\cdot\|_{1}\right)$ and $\left(E,\|\cdot\|_{2}\right)$ are separable Hilbert spaces, and the identity map $E \rightarrow E$ is an FHS-equivalence operator between these Hilbert spaces.

(c) An FHS space is a pair $(G, \mathcal{N})$ of a linear space $G$ (over $\mathbb{R}$, unless it is stated to be complex) and a nonempty set $\mathcal{N}$ of norms on $G$, called admissible norms, such that

every admissible norm turns $G$ into a separable Hilbert space;

all admissible norms are mutually FHS-equivalent;

every norm FHS-equivalent to an admissible norm is also admissible.

(d) An isomorphism of an FHS space $\left(G_{1}, \mathcal{N}_{1}\right)$ to another FHS space $\left(G_{2}, \mathcal{N}_{2}\right)$ is a linear homeomorphism $L: G_{1} \rightarrow G_{2}$ such that $\|L x\|_{2}=\|x\|_{1}$ for some admissible norms $\|\cdot\|_{1}$ on $G_{1}$ and $\|\cdot\|_{2}$ on $G_{2}$.

Every Hilbert space is also an FHS space, and every FHS space is also a linear topological space. Often we say 'an FHS space $G$ ', leaving $\mathcal{N}$ implicit.

A subset $E$ of an FHS space $G$ will be called a (quasi)orthonormal ${ }^{24}$ basis of $G$, if there exists an admissible norm $\|\cdot\|$ on $G$ such that $E$ is an orthonormal basis of the Hilbert space $(G,\|\cdot\|)$.

24 The shorter term 'orthonormal' is unambiguous, as far as $G$ is only an FHS space (that is, no one norm is singled out). Nevertheless, to be on the safe side, I sometimes use the longer term 'quasiorthonormal'. 
8.6 Definition. Let $E, E^{\prime}$ be (quasi)orthonormal bases of an FHS space $G$. A mod 0 isomorphism between measure classes $E^{2}\left(\mathbb{R}^{E}, \mathcal{M}^{E}\right)$ and $\left(\mathbb{R}^{E^{\prime}}, \mathcal{M}^{E^{\prime}}\right)$ is called canonical, if for every $x \in G$, the following two measurable functions (or rather, their equivalence classes) are sent to each other by the isomorphism:

$$
\mathbb{R}^{E} \ni \xi \mapsto \sum_{e \in E}\langle x, e\rangle \xi_{e}, \quad \text { and } \quad \mathbb{R}^{E^{\prime}} \ni \xi \mapsto \sum_{e \in E^{\prime}}\langle x, e\rangle \xi_{e} ;
$$

here, numbers $\langle x, e\rangle$ are defined as the coefficients of the expansion $x=$ $\sum_{e}\langle x, e\rangle e$ (the sum is over $E$ or $E^{\prime}$ respectively).

8.7 Lemma. Let $G$ be an FHS space.

(a) For any two (quasi)orthonormal bases $E, E^{\prime}$ of $G$, a canonical isomorphism between $\left(\mathbb{R}^{E}, \mathcal{M}^{E}\right)$ and $\left(\mathbb{R}^{E^{\prime}}, \mathcal{M}^{E^{\prime}}\right)$ exists and is unique $(\bmod 0)$.

(b) For any three (quasi)orthonormal bases $E, E^{\prime}, E^{\prime \prime}$ of $G$, canonical isomorphisms make the diagram

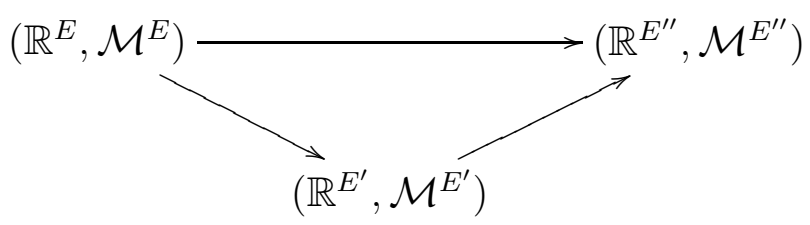

commutative. In addition, the canonical isomorphism $\left(\mathbb{R}^{E}, \mathcal{M}^{E}\right) \rightarrow\left(\mathbb{R}^{E}, \mathcal{M}^{E}\right)$ is the identity map.

The proof is left to the reader.

So, every FHS space $G$ leads to a measure class $\left(X_{G}, \mathcal{M}_{G}\right)$ that is canonically isomorphic $\bmod 0$ to each $\left(\mathbb{R}^{E}, \mathcal{M}^{E}\right)$. 20 We call $\left(X_{G}, \mathcal{M}_{G}\right)$ the standard measure class of $G$. .7 $^{7}$ Each vector $x \in G$ leads to a measurable function $\xi_{x}: X_{G} \rightarrow \mathbb{R}$ (or rather, equivalence class), and the map $x \mapsto \xi_{x}$ is linear. Every admissible norm $\|\cdot\|$ on $G$ leads to a measure $\gamma_{\|\cdot\|}$ on $X_{G}, \gamma_{\|\cdot\|} \in \mathcal{M}_{G}$.

\footnotetext{
${ }^{25}$ As before, $\mathcal{M}^{E}$ is the equivalence class of measures, that contains the product measure $\gamma^{E}$.

${ }^{26}$ More formally: a measurable set $(\bmod 0)$ in $\left(X_{G}, \mathcal{M}_{G}\right)$ is, by definition, a family $A=\left(A_{E}\right)$ such that $A_{E}$ is a measurable set $(\bmod 0)$ in $\left(\mathbb{R}^{E}, \mathcal{M}^{E}\right)$ for every orthonormal basis $E$ of $G$, and for any two such bases, $A_{E_{1}}$ and $A_{E_{2}}$ are sent to each other $(\bmod 0)$ by the canonical isomorphism between $\left(\mathbb{R}^{E_{1}}, \mathcal{M}^{E_{1}}\right)$ and $\left(\mathbb{R}^{E_{2}}, \mathcal{M}^{E_{2}}\right)$. Measurable functions $(\bmod 0)$ on $\left(X_{G}, \mathcal{M}_{G}\right)$ are defined similarly. Note that we do not try to define a point of $X_{G}$ in a canonical way.

${ }^{27}$ Recall that for finite dimension we took $X_{H}=H$. For infinite dimension, $X_{G}$ may be thought of as some extension of the topological dual $G^{\prime}$, and then $G^{\prime}$ is a negligible subset of $X_{G}$.
} 
Such measures will be called Gaussian measures of $\mathcal{M}_{G}$. With respect to the measure $\gamma_{\|\cdot\|}$ we have

$$
\xi_{x} \sim N\left(0,\|x\|^{2}\right) \text { for all } x \in G .
$$

The direct sum of FHS spaces corresponds to the product of their standard measure classes,

$$
\left(X_{G_{1} \oplus G_{2}}, \mathcal{M}_{G_{1} \oplus G_{2}}\right)=\left(X_{G_{1}}, \mathcal{M}_{G_{1}}\right) \times\left(X_{G_{2}}, \mathcal{M}_{G_{2}}\right)
$$

up to a canonical isomorphism.

8.10 Definition. The exponential of an FHS space $G$ over $\mathbb{R}$ is such a Hilbert space (over $\mathbb{R})$ :

$$
e^{G}=L_{2}\left(\left(X_{G}, \mathcal{M}_{G}\right), \mathbb{R}\right)
$$

where $\left(X_{G}, \mathcal{M}_{G}\right)$ is the standard measure class of $G$. Its complexification, $e_{\mathbb{C}}^{G}=L_{2}\left(\left(X_{G}, \mathcal{M}_{G}\right), \mathbb{C}\right)$, is the complex exponential of $G$.

Any isomorphism between FHS spaces, $W: G_{1} \rightarrow G_{2}$, leads to the corresponding isomorphism of measure classes $X_{1} \rightarrow X_{2}$; namely, $\omega_{1} \mapsto \omega_{2}$ means $\xi_{x}\left(\omega_{1}\right)=\xi_{W x}\left(\omega_{2}\right)$. Also, a non-homogeneous linear transformation may be introduced: $\omega_{1} \mapsto \omega_{2}$ means $\xi_{x}\left(\omega_{1}\right)=\xi_{W x}\left(\omega_{2}\right)+\langle x, y\rangle$, where $y \in G_{1}^{\prime}$ is a given linear functional on $G_{1}$. The isomorphism of measure classes induces a unitary operator $\Gamma(W, y)=U: e^{G_{1}} \rightarrow e^{G_{2}}$ between Hilbert spaces; namely, $U\left(f_{1} \sqrt{\mu_{1}}\right)=f_{2} \sqrt{\mu_{2}}$ whenever $f_{1}\left(\omega_{1}\right)=f_{2}\left(\omega_{2}\right)$ and $\mu_{1}\left(d \omega_{1}\right)=\mu_{2}\left(d \omega_{2}\right)$. Note that $\Gamma(\mathbf{1}, y) \Gamma(W, 0)=\Gamma\left(W, W^{*} y\right)=\Gamma(W, 0) \Gamma\left(\mathbf{1}, W^{*} y\right)$; of course, $\left\langle x, W^{*} y\right\rangle=$ $\langle W x, y\rangle$.

8.11 Lemma. (a) $e^{G_{1} \oplus G_{2}}=e^{G_{1}} \otimes e^{G_{2}}$ (up to canonical isomorphism), for any FHS spaces $G_{1}, G_{2}$.

(b) The group of automorphisms of an FHS space $G$ acts on $e^{G}$ by unitary operators, $W \mapsto \Gamma(W)$.

(c) The additive group of the (topological) dual space $G^{\prime}$ of an FHS space $G$ acts on $e^{G}$ (and $e_{\mathbb{C}}^{G}$ ) by unitary operators $V_{y}$ such that $V_{y} \Gamma(W)=$ $\Gamma(W) V_{W^{*} y}$; the additive group of $G$ acts on $e_{\mathbb{C}}^{G}$ by unitary operators $U_{x}$; these operators satisfy Weil relations, $U_{x} V_{y}=e^{-i\langle x, y\rangle} V_{y} U_{x}$. For each Gaussian measure $\gamma \in \mathcal{M}_{G}$ we have $\left\langle V_{y} \sqrt{\gamma}, \sqrt{\gamma}\right\rangle=\exp \left(-\frac{1}{8}\|y\|^{2}\right)$ and $\left\langle U_{x} \sqrt{\gamma}, \sqrt{\gamma}\right\rangle=$ $\exp \left(-\frac{1}{2}\|x\|^{2}\right)$ for all $x \in G, y \in G^{\prime}$; here $\|\cdot\|$ is the admissible norm on $G$ corresponding to $\gamma$ (or its dual norm on $G^{\prime}$ ). Also, $e^{G}$ is spanned by $\left\{U_{x} \sqrt{\gamma}: x \in G\right\}$, as well as by $\left\{V_{y} \sqrt{\gamma}: y \in G^{\prime}\right\}$.

(d) Let $G$ be an FHS space, $(X, \mathcal{B}, \mathcal{M})$ a measure class, $\gamma \in \mathcal{M}$ a probability measure, and $\tilde{\eta}: G \rightarrow L_{2}(\gamma)$ a linear map, isometric from $(G,\|\cdot\|)$ 
to $L_{2}(\gamma)$ for some admissible norm $\|\cdot\|$ on $G$, and each $\tilde{\eta}(x)$ has the normal distribution $N\left(0,\|x\|^{2}\right)$ w.r.t. $\gamma$, and the image $\tilde{\eta}(G) \subset L_{2}(\gamma)$ generates the whole $\sigma$-field $\mathcal{B}$. Then there exists one and only one isomorphism of measure classes $\eta:\left(X_{G}, \mathcal{M}_{G}\right) \rightarrow(X, \mathcal{M})$ inducing $\tilde{\eta}$ by $\tilde{\eta}(x)(\omega)=x\left(\eta^{-1} \omega\right)$ for $\omega \in X$, $x \in G$.

The proof is left to the reader.

The next result implies that the obstacle mentioned in Remark 3.9 does not appear when dealing with Gaussian measures. Its natural domain is linear topological spaces, but we need only FHS spaces. See also Remark 9.1 .

8.12 Lemma. Let $G$ be a linear topological space and $G_{1}, G_{1,2}, G_{2,3}, G_{3}$ its (closed linear) subspaces such that $G_{1} \subset G_{1,2}, G_{2,3} \supset G_{3}$, and

$$
G_{1,2} \oplus G_{3}=G=G_{1} \oplus G_{2,3}
$$

Then the subspace $G_{2}=G_{1,2} \cap G_{2,3}$ satisfies

$$
G_{1} \oplus G_{2} \oplus G_{3}=G, \quad G_{1} \oplus G_{2}=G_{1,2}, \quad G_{2} \oplus G_{3}=G_{2,3} .
$$

(Direct sums are treated in the topological sense.)

Proof. The decomposition $G=G_{1} \oplus G_{2,3}$ determines a projection $P_{1}: G \rightarrow G$ such that $P_{1} G=G_{1}$ and $1-P_{1}=P_{2,3}$ is also a projection, $P_{2,3} G=G_{2,3}$. The same for $P_{3}$ and $P_{1,2}=1-P_{3}$. The inclusion $G_{1} \subset G_{1,2}$ gives $P_{1}=P_{1,2} P_{1}=$ $\left(1-P_{3}\right) P_{1}$, that is, $P_{3} P_{1}=0$; similarly, $P_{1} P_{3}=0$. So, our projections commute with each other. Introducing

$$
P_{2}=P_{12} P_{23}=P_{23} P_{12}=\left(1-P_{1}\right)\left(1-P_{3}\right)=1-P_{1}-P_{3},
$$

we have $P_{2}^{2}=P_{1,2} P_{2,3} P_{1,2} P_{2,3}=P_{1,2}^{2} P_{2,3}^{2}=P_{1,2} P_{2,3}=P_{2}$; that is, $P_{2}$ is also a projection, and $P_{1}+P_{2}+P_{3}=1$. It follows that $P_{2} G=\left(\left(P_{1}+P_{2}\right) G\right) \cap$ $\left(\left(P_{2}+P_{3}\right) G\right)=G_{1,2} \cap G_{2,3}=G_{2}$.

\section{Continuous sums and off-white noises}

Roughly speaking, a continuous sum of FHS spaces is a family $\left(G_{s, t}\right)_{s<t}$ of FHS spaces $G_{s, t}$ such that $G_{r, s} \oplus G_{s, t}=G_{r, t}$. One can turn the idea into a definition in the style of 1.5 (and 3.2) by introducing isomorphisms $W_{r, s, t}: G_{r, s} \oplus G_{s, t} \rightarrow G_{r, t}$ of FHS spaces. However, I prefer an equivalent definition in the style of 3.10 . 
A (closed linear) subspace $G_{1}$ of an FHS space $G$ inherits from $G$ the structure of an FHS space. Given two subspaces $G_{1}, G_{2} \subset G$, we may consider the map $G_{1} \oplus G_{2} \ni x \oplus y \mapsto x+y \in G$ and ask whether it is an isomorphism of FHS spaces, or not. If it is, we say that $G_{1}, G_{2}$ are (quasi)orthogonal (see [A, Sect. 3.3.3], А+, Sect. 14.1.1]) and write ' $G=G_{1} \oplus G_{2}$ in the FHS sense'. Do not confuse it with the relation ' $G=G_{1} \oplus G_{2}$ in the topological sense'; the latter means that the map $G_{1} \oplus G_{2} \ni x \oplus y \mapsto x+y \in G$ is a linear homeomorphism, but not necessarily an isomorphism of FHS spaces. Similarly, the relation ' $G=G_{1} \oplus G_{2} \oplus G_{3}$ in the FHS sense' means, by definition, that the map $G_{1} \oplus G_{2} \oplus G_{3} \ni x \oplus y \oplus z \mapsto x+y+z \in G$ is an isomorphism of FHS spaces.

9.1 Remark. Let $G$ be an FHS space and $G_{1}, G_{1,2}, G_{2,3}, G_{3}$ its subspaces such that $G_{1} \subset G_{1,2}, G_{2,3} \supset G_{3}$, and

$$
G_{1,2} \oplus G_{3}=G=G_{1} \oplus G_{2,3} \quad \text { in the FHS sense. }
$$

Then the subspace $G_{2}=G_{1,2} \cap G_{2,3}$ satisfies

$G_{1} \oplus G_{2} \oplus G_{3}=G, \quad G_{1} \oplus G_{2}=G_{1,2}, \quad G_{2} \oplus G_{3}=G_{2,3} \quad$ in the FHS sense.

Indeed, we apply Lemma 8.12 and combine FHS isomorphisms $G_{1} \oplus$ $G_{2,3} \rightarrow G$ and $G_{2} \oplus G_{3} \rightarrow G_{2,3}$ into $G_{1} \oplus G_{2} \oplus G_{3} \rightarrow G_{1} \oplus G_{2,3} \rightarrow G$.

9.2 Definition. A continuous sum of FHS spaces is $\left(G,\left(G_{s, t}\right)_{-\infty \leq s<t \leq \infty}\right)$ where $G$ is an FHS space, and $G_{s, t} \subset G$ are subspaces such that

$$
G_{r, s} \oplus G_{s, t}=G_{r, t} \quad \text { whenever }-\infty \leq r<s<t \leq \infty
$$

and $G_{-\infty, \infty}=G$.

9.3 Corollary. If $\left(G,\left(G_{s, t}\right)_{s<t}\right)$ is a continuous sum of FHS spaces, then $\left(e^{G_{s, t}}\right)_{s<t}$ is a real CP HS, and $\left(e_{\mathbb{C}}^{G_{s, t}}\right)_{s<t}$ is a (complex) CP HS.

(To be rigorous, canonical isomorphisms $W_{r, s, t}: e^{G_{r, s}} \otimes e^{G_{s, t}} \rightarrow e^{G_{r, s} \oplus G_{s, t}} \rightarrow$ $e^{G_{r, t}}$ should be included.)

The transition from the continuous sum to the CP HS is mediated by a CP MC. Namely, $e^{G_{s, t}}=L_{2}\left(\left(X_{s, t}, \mathcal{M}_{s, t}\right), \mathbb{R}\right)$ and $e_{\mathbb{C}}^{G_{s, t}}=L_{2}\left(\left(X_{s, t}, \mathcal{M}_{s, t}\right), \mathbb{C}\right)$, where $\left(X_{s, t}, \mathcal{M}_{s, t}\right)$ is the standard measure class of $G_{s, t} ;$ and $\left(X_{r, s}, \mathcal{M}_{r, s}\right) \times$ $\left(X_{s, t}, \mathcal{M}_{s, t}\right)=\left(X_{r, t}, \mathcal{M}_{r, t}\right)$ by $(8.9)$. Alternatively, we may treat the CP MC according to 3.10 (rather than 3.2); that is, on the standard measure class

\footnotetext{
28 Similarly to Footnote 24, the shorter term is unambiguous, but the longer term is safer.
} 
$(X, \mathcal{B}, \mathcal{M})$ of $G$ we have sub- $\sigma$-fields $\mathcal{B}_{s, t}$ such that $\mathcal{B}_{r, s} \otimes \mathcal{B}_{s, t}=\mathcal{B}_{r, t}$. Namely, $\mathcal{B}_{s, t}$ is generated by measurable functions $\xi_{x}, x \in G_{s, t}$. A similar transition from a homogeneous continuous sum of FHS spaces to a HCP HS via a HCP $\mathrm{MC}$ is discussed below.

9.4 Definition. A homogeneous continuous sum of FHS spaces is $\left(G,\left(G_{s, t}\right)_{-\infty \leq s<t \leq \infty},\left(\tilde{\theta}^{u}\right)_{u \in \mathbb{R}}\right)$ where $\left(G,\left(G_{s, t}\right)_{-\infty \leq s<t \leq \infty}\right)$ is a continuous sum of FHS spaces, and $\left(\tilde{\theta}^{u}\right)_{u \in \mathbb{R}}$ is a one-parameter group of automorphisms $\tilde{\theta}^{u}$ of the FHS space $G$ such that

$$
\tilde{\theta}^{u}\left(G_{s, t}\right)=G_{s+u, t+u} \quad \text { whenever }-\infty \leq s<t \leq \infty, u \in \mathbb{R},
$$

and the corresponding unitary group $\theta^{u}=\Gamma\left(\tilde{\theta}^{u}\right)$ on $e^{G}$ is strongly continuous.

No need to elaborate on the latter condition, since all we need is such a simple sufficient condition:

there exists an admissible norm $\|\cdot\|$ on $G$ such that $\left(\tilde{\theta}^{u}\right)_{u \in \mathbb{R}}$ is a strongly continuous unitary group on the Hilbert space $(G,\|\cdot\|)$.

Indeed, it implies that $\theta^{u} \sqrt{\gamma}=\sqrt{\gamma}$ (here $\gamma=\gamma_{\|\cdot\|}$ ) and $\theta^{u} V_{y} \sqrt{\gamma}=$ $V_{\left(\tilde{\theta}^{-u}\right)^{*}} \theta^{u} \sqrt{\gamma} \rightarrow V_{y} \sqrt{\gamma}$ when $u \rightarrow 0$, for each $y \in G^{\prime}$; here $\theta^{u}=\Gamma\left(\tilde{\theta}^{u}\right)$. Taking into account that the set $\left\{V_{y} \sqrt{\gamma}: y \in G^{\prime}\right\}$ spans $e^{G}$ we conclude that $\theta^{u} \rightarrow 1$ strongly on $e^{G}$ when $u \rightarrow 0$.

Let $\left(G,\left(G_{s, t}\right)_{s<t},\left(\tilde{\theta}^{u}\right)_{u \in \mathbb{R}}\right)$ be a homogeneous continuous sum of FHS spaces, and $\left(e^{G_{s, t}}\right)_{s<t}$ the corresponding real $\mathrm{CP}$ HS. The restriction $\tilde{\theta}_{s, t}^{u}$ of $\theta^{u}$ to $G_{s, t}$, being an isomorphism $\tilde{\theta}_{s, t}^{u}: G_{s, t} \rightarrow G_{s+u, t+u}$ of FHS spaces, leads to the corresponding unitary operator $\theta_{s, t}^{u}: e^{G_{s, t}} \rightarrow e^{G_{s+u, t+u}}$. Clearly, $\tilde{\theta}_{r, s}^{u} \oplus \tilde{\theta}_{s, t}^{u}=\tilde{\theta}_{r, t}^{u}$, which ensures 1.6(a1). Condition 1.6(a2) follows from the relation $\tilde{\theta}^{v} \tilde{\theta}^{u}=\tilde{\theta}^{u+v}$. Condition 1.6(a3) is the strong continuity.

9.6 Corollary. If $\left(G,\left(G_{s, t}\right)_{s<t},\left(\tilde{\theta}^{u}\right)_{u \in \mathbb{R}}\right)$ is a homogeneous continuous sum of FHS spaces, then $\left(\left(e^{G_{s, t}}\right)_{s<t},\left(\theta_{s, t}^{u}\right)_{s<t, u \in \mathbb{R}}\right)$ is a real HCP HS, and its complexification is a (complex) HCP HS.

In combination with Theorem 1.9 it means that each homogeneous continuous sum of FHS spaces leads to a product system.

The simplest nontrivial example of a homogeneous continuous sum of FHS spaces is given by $G_{s, t}=L_{2}(s, t) \subset L_{2}(\mathbb{R})$. These are Hilbert spaces, and $G_{r, s} \oplus G_{s, t}=G_{r, t}$ in the Hilbert-space sense, which is much better than needed. The corresponding Gaussian measure describes the white noise; the HCP MC is in fact an HCP PS, and the product system is of type $I$. 
In order to get more interesting product systems, we modify the metric of $L_{2}(\mathbb{R})$, keeping it shift-invariant. The idea is to define $\|f\|_{C}^{2}=$ $\iint f(s) f(t) C(s-t) d s d t$; however, technically, it is better to use the Fourier transform, $\hat{f}(\lambda)=(2 \pi)^{-1 / 2} \int f(t) e^{-i \lambda t} d t$, and define

$$
\|f\|_{\nu}^{2}=\int|\hat{f}(\lambda)|^{2} \nu(d \lambda)
$$

The idea is implemented as follows.

Let $\nu$ be a positive $\sigma$-finite measure on $[0, \infty)$ such that $\int\left(1+\lambda^{2}\right)^{-m} \nu(d \lambda)<$ $\infty$ for $m$ large enough. We exclude the trivial case $\nu=0$, and consider the Hilbert space $L_{2}(\nu)$, and its subspace $G$ spanned by functions $\hat{f}$ when $f$ runs over all compactly supported functions $f: \mathbb{R} \rightarrow \mathbb{R}$ of class $C^{2 m}$ (in fact, $G$ is the whole $\left.L_{2}(\nu)\right)$. Requiring $f$ to vanish on $(t, \infty)$ we get a subspace $G_{-\infty, t}$ of $G$. Similarly, $G_{t, \infty}$ is spanned by $\hat{f}$ for $f$ vanishing on $(-\infty, t)$. Despite the complex nature of $L_{2}(\nu)$, we downgrade $G$ to a Hilbert space over $\mathbb{R}$ and further, to a real FHS space. Only the case $m=1$ is important for us (see Ts02 for the general case).

9.7 Theorem. [Ts02, Th. 3.2]. The following two conditions on $\nu$ are equivalent:

(a) $G=G_{-\infty, 0} \oplus G_{0, \infty}$ in the FHS sense, and $\int\left(1+\lambda^{2}\right)^{-1} \nu(d \lambda)<\infty$;

(b) $\nu(d \lambda)=e^{\varphi(\lambda)} d \lambda$ for some $\varphi:[0, \infty) \rightarrow \mathbb{R}$ such that

$$
\int_{0}^{\infty} \int_{0}^{\infty} \frac{\left|\varphi\left(\lambda_{1}\right)-\varphi\left(\lambda_{2}\right)\right|^{2}}{\left|\lambda_{1}-\lambda_{2}\right|^{2}} d \lambda_{1} d \lambda_{2}<\infty .
$$

9.9 Lemma. If $\nu$ satisfies the (equivalent) conditions of Theorem 9.7, then subspaces $G_{s, t}=G_{-\infty, t} \cap G_{s, \infty}$ of $G$ form a continuous sum of FHS spaces.

Proof. First, $G_{r, s} \oplus G_{s, t}=G_{r, t}$ in the topological sense, which is proven similarly to Lemma 8.12. Second, $G_{r, s}$ and $G_{s, t}$ are quasi-orthogonal, since $G_{-\infty, s}$ and $G_{s, \infty}$ are.

Unitary operators $\tilde{\theta}^{u}: L_{2}(\nu) \rightarrow L_{2}(\nu)$ defined by $\tilde{\theta}^{u} g(\lambda)=e^{-i u \lambda} g(\lambda)$ satisfy $\tilde{\theta}^{u}\left(G_{s, t}\right)=G_{s+u, t+u}$ and (9.5). Therefore $\left(G,\left(G_{s, t}\right)_{s<t},\left(\tilde{\theta}^{u}\right)_{u \in \mathbb{R}}\right)$ is a homogeneous continuous sum of FHS spaces, and by Corollary 9.6, Hilbert spaces

$$
H_{s, t}=e^{G_{s, t}}
$$

form a real HCP HS (which leads to a product system).

The theory of random processes can contribute to our understanding of the spaces $G$ and $H=e^{G}$. It is well-known that every positive $\sigma$-finite measure $\nu$ on $[0, \infty)$ such that $\int\left(1+\lambda^{2}\right)^{-m} \nu(d \lambda)<\infty$ (for $m$ large enough) is the 
spectral measure of a stationary Gaussian generalized random process $\xi(\cdot)$. In other words, there exists one and only one shift-invariant Gaussian measure $\gamma_{\nu}$ on the space $\Omega$ of (tempered, Schwartz; real-valued) distributions 20 (generalized functions) over $\mathbb{R}$ such that

$$
\int_{\Omega}\left(\int_{\mathbb{R}} f(t) \xi(t) d t\right)^{2} \gamma_{\nu}(d \xi)=\|\hat{f}\|_{L_{2}(\nu)}^{2}
$$

for every compactly supported $f$ of class $C^{2 m}$. More probabilistically,

$$
\int_{\mathbb{R}} f(t) \xi(t) d t \sim N\left(0,\|\hat{f}\|_{L_{2}(\nu)}^{2}\right) .
$$

Lemma 8.11(d) gives us a canonical isomorphism between two measure classes, the standard measure class $\left(X_{G}, \mathcal{M}_{G}\right)$ of the FHS space $G$ and $\left(\Omega, \mathcal{M}_{\nu}\right)$, where $\mathcal{M}_{\nu}$ is the equivalence class containing the measure $\gamma_{\nu}$. We treat them as identical:

$$
\left(X_{G}, \mathcal{M}_{G}\right)=\left(\Omega, \mathcal{M}_{\nu}\right) ; \quad H=L_{2}\left(\left(\Omega, \mathcal{M}_{\nu}\right), \mathbb{R}\right)=L_{2}\left(\left(\Omega, \gamma_{\nu}\right), \mathbb{R}\right) ;
$$

the random variable $\int f(t) \xi(t) d t$ is nothing but $\xi_{x}$ (as in (8.8)) for $x=f$. However, smooth test functions $f$ represent continuous linear functionals on $\Omega$, while points $x$ of $G$ represent measurable linear functionals on $\Omega$. These $f$ are dense in $G$, and $f$ that vanish on $(-\infty, 0)$ are dense in $G_{0, \infty}$.

If $\nu$ satisfies equivalent conditions of Theorem 9.7, then the random process $\xi(\cdot)$ is a so-called off-white noise. Its past (the restriction of $\xi(\cdot)$ to $(-\infty, 0))$ and future (the restriction of $\xi(\cdot)$ to $(0, \infty))$ are stochastically dependent (unless the noise is white), but not too much; the product of marginal distributions is equivalent to the given distribution.

From now on we restrict ourselves to measures $\nu$ that satisfy equivalent conditions of Theorem 9.7 .

The function $e^{\varphi(\lambda)}=\nu(d \lambda) / d \lambda$ will be called the spectral density. Condition 9.7(b) ensures that $\nu(d \lambda) / d \lambda>0$ almost everywhere; thus $\nu$ is equivalent to the Lebesgue measure.

A sufficient condition for (9.8) is available (see [Ts02, Prop. 3.6(b)]):

$$
\varphi \text { is continuously differentiable, and } \int_{0}^{\infty}\left|\frac{d}{d \lambda} \varphi(\lambda)\right|^{2} \lambda d \lambda<\infty .
$$

\footnotetext{
${ }^{29}$ Sorry, 'a distribution in the space of distributions' may be confusing. A 'probability distribution' is just a probability measure (intended to describe a random element of the corresponding space). In contrast, a generalized function, also called 'distribution', is a more singular (than a measure) object over $\mathbb{R}$, generally not positive; for example, a derivative $\delta^{(n)}$ of Dirac's delta-function.

${ }^{30}$ In fact, $\int\left(\frac{\nu(d \lambda)}{d \lambda}\right)^{p} \frac{d \lambda}{1+\lambda^{2}}<\infty$ for all $p \in(-\infty, \infty)$, but we do not need it.
} 
In particular, the sufficient condition is satisfied by any strictly positive smooth function $\lambda \mapsto e^{\varphi(\lambda)}=\nu(d \lambda) / d \lambda$ such that for $\lambda$ large enough, one of the following equalities holds:

$$
\begin{gathered}
\frac{\nu(d \lambda)}{d \lambda}=\ln ^{\alpha} \lambda, \quad-\infty<\alpha<\infty \\
\frac{\nu(d \lambda)}{d \lambda}=\exp \left(-\ln ^{\alpha} \lambda\right), \quad 0<\alpha<\frac{1}{2}
\end{gathered}
$$

see [Ts02, Examples 3.11, 3.12].

The case of $\ln ^{\alpha} \lambda$ with $\alpha>0$ shows that the spectral density of an offwhite noise need not be bounded. From now on we assume boundedness (in addition to $9.7(\mathrm{~b})):$ 阝

$$
\sup _{\lambda \in[0, \infty)} \frac{\nu(d \lambda)}{d \lambda}<\infty
$$

(Thus, $\ln ^{\alpha} \lambda$ fits only for $-\infty<\alpha \leq 0$.) It follows that $\|\hat{f}\|_{L_{2}(\nu)} \leq$ const $\cdot\|\hat{f}\|_{L_{2}(0, \infty)}=$ const $\cdot\|f\|_{L_{2}(\mathbb{R})}$. We have a continuous operator $\iota$ : $L_{2}(\mathbb{R}) \rightarrow G$

$$
\begin{gathered}
G \text { is the closure of } \iota\left(L_{2}(\mathbb{R})\right) ; \\
G_{-\infty, t} \text { is the closure of } \iota\left(L_{2}(-\infty, t)\right) ; \\
G_{s, \infty} \text { is the closure of } \iota\left(L_{2}(s, \infty)\right) .
\end{gathered}
$$

In contrast to $C^{2 m}$, the space $L_{2}(\mathbb{R})$ is closed under multiplication by the indicator $\mathbf{1}_{(s, t)}$ of an interval $(s, t) \subset \mathbb{R}$. Projections $Q_{-\infty, t}$ and $Q_{t, \infty}=$ $1-Q_{-\infty, t}$ on $G$, corresponding to the decomposition $G=G_{-\infty, t} \oplus G_{t, \infty}$, satisfy

$$
\begin{array}{r}
Q_{-\infty, t} \iota(f)=\iota\left(f \cdot \mathbf{1}_{(-\infty, t)}\right) ; \\
Q_{s, \infty} \iota(f)=\iota\left(f \cdot \mathbf{1}_{(s, \infty)}\right) .
\end{array}
$$

Therefore the projection $Q_{s, t}=Q_{-\infty, t} Q_{s, \infty}=Q_{s, \infty} Q_{-\infty, t}$ satisfies

$$
Q_{s, t} \iota(f)=\iota\left(f \cdot \mathbf{1}_{(s, t)}\right)
$$

which implies that

$$
G_{s, t} \text { is the closure of } \iota\left(L_{2}(s, t)\right) .
$$

\footnotetext{
${ }^{31}$ After an appropriate change of $\nu(d \lambda) / d \lambda$ on a negligible set, of course.

${ }^{32}$ By $L_{2}(\mathbb{R})$ I mean the space of real-valued (not complex-valued) functions on $\mathbb{R}$.
} 
The random variable $\xi_{x}$ (recall (8.8)) corresponding to $x=\iota(f)$ where $f \in$ $L_{2}(\mathbb{R})$ will be denoted by

$$
\int_{-\infty}^{\infty} f(t) \xi(t) d t
$$

Now, (9.10) holds for all $f \in L_{2}(\mathbb{R})$. When $f$ is good enough, the new meaning of $\int f(t) \xi(t) d t$ conforms $\gamma_{\nu}$-almost everywhere to the meaning used in (9.10). Of course, $\int_{s}^{t} f(u) \xi(u) d u$ stands for $\int_{-\infty}^{\infty}\left(f(u) \mathbf{1}_{(s, t)}(u)\right) \xi(u) d u$. Note that $\int_{s}^{t} f(u) \xi(u) d u$ is measurable w.r.t. the sub- $\sigma$-field $\mathcal{B}_{s, t}$ corresponding to $G_{s, t}$ (these sub- $\sigma$-fields form a CP MC). Also, $\mathcal{B}_{s, t}$ is generated by $\left\{\int_{s}^{t} f(u) \xi(u) d u: f \in L_{2}(s, t)\right\}$.

\section{Type $I I I$}

We still do not know a "natural" construction of a product system without any units... We believe that there should be a natural way of constructing such product systems, and we offer that as a basic unsolved problem.

W. Arveson A97, Introduction]

We consider the homogeneous continuous sum of FHS spaces constructed in Sect. 9 for a spectral density that satisfies 9.7(b), (9.14) and

$$
\frac{\nu(d \lambda)}{d \lambda} \rightarrow 0 \text { for } \lambda \rightarrow \infty
$$

(the white noise is now excluded). It will be proven that the corresponding product system is of type $I I I$. Recall that $\|f\|_{\nu}=\|\hat{f}\|_{L_{2}(\nu)}$ for $f \in L_{2}(\mathbb{R})$.

10.2 Lemma. Let $f \in L_{2}(\mathbb{R})$. Define $f_{n} \in L_{2}(\mathbb{R})$ by

$$
f_{n}(t)=f(t) \cdot \operatorname{sgn} \sin \pi n t= \begin{cases}f(t) & \text { for } t \in \cup_{k \in \mathbb{Z}}\left(\frac{2 k}{n}, \frac{2 k+1}{n}\right), \\ -f(t) & \text { otherwise }\end{cases}
$$

Then $\left\|f_{n}\right\|_{\nu} \rightarrow 0$ for $n \rightarrow \infty$.

Proof. Taking into account that $\|\cdot\|_{\nu} \leq$ const $\cdot\|\cdot\|$ (here $\|\cdot\|$ is the usual norm of $L_{2}(\mathbb{R})$ ), we may restrict ourselves to $f$ of a dense subset of $L_{2}(\mathbb{R})$. Thus, we assume that $\hat{f}$ has a compact support.

The Fourier transform of the periodic function $t \mapsto \operatorname{sgn} \sin \pi n t$ is concentrated on the (shifted) lattice $\pi n(1+2 \mathbb{Z})=\{\ldots,-3 \pi n,-\pi n, \pi n, 3 \pi n, \ldots\}$. Let $n$ be large enough, such that the interval $[-\pi n, \pi n]$ contains the support 
of $\hat{f}$. Then $\hat{f}_{n}$ consists of non-overlapping shifted copies of $\hat{f}$, multiplied by Fourier coefficients of the periodic function. We have

$$
\left\|f_{n}\right\|_{\nu}^{2}=\int\left|\hat{f}_{n}(\lambda)\right|^{2} \nu(d \lambda) d \lambda \leq \mathrm{const} \cdot\|\hat{f}\|^{2} \cdot \sup _{\lambda \in \operatorname{supp} \hat{f}_{n}} \frac{\nu(d \lambda)}{d \lambda} \rightarrow 0
$$

indeed, for large $n$, the support of $\hat{f}_{n}$ is distant from the origin.

The following result holds for real and complex CPs HS equally well.

10.3 Lemma. The CP HS over $[0,1]$, corresponding to the considered continuous sum of FHS spaces, is of type $I I I$.

Proof. The CP HS arises from a CP MC formed by sub- $\sigma$-fields $\left(\mathcal{B}_{s, t}\right)_{0 \leq s<t \leq 1}$ on the measure class $\left(\Omega, \mathcal{M}_{\nu}\right)$. We choose $f \in L_{2}(0,1)$ such that $\|f\|_{\nu} \neq 0$ (in fact, every $f \neq 0$ fits, since $\nu$ is equivalent to the Lebesgue measure). With respect to the Gaussian measure $\gamma_{\nu}$, the random variable $\int_{0}^{1} f(u) \xi(u) d u$ has a non-degenerate normal distribution. Therefore $\int_{0}^{1} f(u) \xi(u) d u$ has an absolutely continuous distribution for every probability measure of $\mathcal{M}_{\nu}$, and moreover, for every probability measure absolutely continuous w.r.t. $\mathcal{M}_{\nu}$.

Assume that the CP HS is not of type $I I I$, and let $\psi \in H_{0,1}$ be a decomposable vector, $\|\psi\|=1$. The probability measure $\mu=|\psi|^{2}$ on $\left(\Omega, \mathcal{B}_{0,1}\right)$ is absolutely continuous w.r.t. $\mathcal{M}$, and makes sub- $\sigma$-fields $\mathcal{B}_{r, s}$ and $\mathcal{B}_{s, t}$ independent whenever $0 \leq r<s<t \leq 1$ (see Lemma 4.1). Therefore it makes independent the following two random variables (for each $n$ separately):

$$
\int_{0}^{1}\left(f(u)-f_{n}(u)\right) \xi(u) d u \text { and } \int_{0}^{1}\left(f(u)+f_{n}(u)\right) \xi(u) d u,
$$

where $f_{n}$ are as in Lemma 10.2. (Indeed, it makes the $n$ sub- $\sigma$-fields $\mathcal{B}_{k / n,(k+1) / n}$ independent.)

By Lemma 10.2 and (9.10), measurable functions $\int_{0}^{1} f_{n}(u) \xi(u) d u$ converge to 0 (for $n \rightarrow \infty$ ) in $L_{2}\left(\gamma_{\nu}\right)$, therefore, in measure w.r.t. $\gamma_{\nu}$, and therefore, also in measure w.r.t. $\mu$. So, w.r.t. $\mu$ the random variable

$\int_{0}^{1} f(u) \xi(u) d u=\lim _{n} \int_{0}^{1}\left(f(u)-f_{n}(u)\right) \xi(u) d u=\lim _{n} \int_{0}^{1}\left(f(u)+f_{n}(u)\right) \xi(u) d u$

is independent of itself! It means that the random variable is degenerate (constant), which is impossible, since its distribution is absolutely continuous. 


\section{The invariant via the logarithm}

Infinitesimality, as defined by Definition 2.2, will be used as an invariant for discriminating CPs HS of type III that emerge by exponentiation from continuous sums of FHS spaces. To this end, infinitesimality w.r.t. such a CP HS will be translated into the language of FHS spaces.

11.1 Definition. Let $X$ be a metrizable topological space and $X_{1}, X_{2}, \ldots \subset$ $X$ closed subsets. We define $\liminf _{n \rightarrow \infty} X_{n}$ as the set of limits of all convergent sequences $x_{1}, x_{2}, \ldots$ such that $x_{1} \in X_{1}, x_{2} \in X_{2}, \ldots$

The set $\lim \inf X_{n}$ is always closed. If $X$ is a linear topological space and $X_{n}$ are linear subspaces, then $\lim \inf X_{n}$ is a linear subspace. The following definition is formulated for linear topological spaces, but we need it only for FHS spaces.

11.2 Definition. Let $G$ be a linear topological space and $G_{1}, G_{2}, \ldots$ its (closed linear) subspaces. We say that $\lim \sup G_{n}=\{0\}$, if $f\left(x_{n}\right) \rightarrow 0$ for every bounded sequence $\left(x_{n}\right)$ such that $x_{n} \in G_{n}$, and every (continuous) linear functional $f$ on $G$.

Let $G$ be an FHS space and $E_{n}, F_{n}$ its subspaces such that $G=E_{n} \oplus F_{n}$ (in the FHS sense) for each $n$. We have the tensor product of Hilbert spaces $e^{E_{n}} \otimes e^{F_{n}}=e^{G}$, and two factors $\mathcal{A}_{n}=\mathbf{1} \otimes \mathcal{B}\left(e^{F_{n}}\right), \mathcal{A}_{n}^{\prime}=\mathcal{B}\left(e^{E_{n}}\right) \otimes \mathbf{1}$ in the operator algebra $\mathcal{A}=\mathcal{B}\left(e^{G}\right)$. These are real, but may be complexified. Real-valued and complex-valued versions of Condition (a) of the following theorem are equivalent, due to (an evident generalization of) Lemma 2.11. We concentrate on the real case until Lemma 11.9.

11.3 Theorem. The following two conditions are equivalent.

(a) $\sup _{A \in \mathcal{A}_{n},\|A\| \leq 1}|\operatorname{tr}(A R)| \rightarrow 0$ for $n \rightarrow \infty$ for each trace-class operator $R \in \mathcal{B}\left(e^{G}\right)$ satisfying $\operatorname{tr}(R)=0$;

(b) $\liminf E_{n}=G$ and $\limsup F_{n}=\{0\}$.

The proof will be given after several lemmas and definitions.

Let $G$ be an FHS space and $(X, \mathcal{B}, \mathcal{M})$ its standard measure class. Admissible norms on $G$ are in a natural one-one correspondence with Gaussian measures of $\mathcal{M},\|\cdot\| \leftrightarrow \gamma_{\|\cdot\|}$. Moreover, the correspondence is a homeomorphism, if both sets are equipped with appropriate topologies. For any two admissible norms $\|\cdot\|_{1},\|\cdot\|_{2}$ there exists a basis $\left(e_{k}\right)$ of $G$, orthogonal for both norms, and eigenvalues $\lambda_{k}=\left\|e_{k}\right\|_{2} /\left\|e_{k}\right\|_{1}$ satisfy $\sum\left(\lambda_{k}^{2}-1\right)^{2}<\infty$ (recall 
8.5(a); $1-\lambda_{k}^{2}$ are eigenvalues of $\left.1-L^{*} L\right)$, or equivalently, $\sum\left(\lambda_{k}-1\right)^{2}<\infty$. A simple (basically, one-dimensional) calculation gives

$$
\left\langle\sqrt{\gamma_{1}}, \sqrt{\gamma_{2}}\right\rangle=\prod_{k}\left(\frac{\lambda_{k}^{-1 / 2}+\lambda_{k}^{1 / 2}}{2}\right)^{-1 / 2}
$$

where $\gamma_{1}, \gamma_{2}$ correspond to $\|\cdot\|_{1},\|\cdot\|_{2}$. Of course, convergence of the product is equivalent to convergence of the series $\sum_{k}\left(\lambda_{k}-1\right)^{2}$. We may use, say, $\arccos \left\langle\sqrt{\gamma_{1}}, \sqrt{\gamma_{2}}\right\rangle$ as the distance between $\gamma_{1}$ and $\gamma_{2}$, and $\arccos \prod\left(\frac{\lambda_{k}^{-1 / 2}+\lambda_{k}^{1 / 2}}{2}\right)^{-1 / 2}$ as the distance between $\|\cdot\|_{1}$ and $\|\cdot\|_{2}{ }^{\text {B3 }}$ which makes the correspondence isometric. The distance defines a topology on the set of all admissible norms. (No other topology will be used on this set.) The distance $\arccos \left\langle\sqrt{\gamma_{1}}, \sqrt{\gamma_{2}}\right\rangle$ on the set of Gaussian measures of $\mathcal{M}$ is equivalent to the variation distance $\left\|\gamma_{1}-\gamma_{2}\right\|=\int\left|\gamma_{1}(d \omega)-\gamma_{2}(d \omega)\right|$. The same holds for arbitrary (not just Gaussian) probability measures of $\mathcal{M}$; namely, $2-\sqrt{4-\|\mu-\nu\|^{2}} \leq\|\sqrt{\mu}-\sqrt{\nu}\|^{2} \leq\|\mu-\nu\|$. The variation distance defines a topology on the set of all probability measures of $\mathcal{M}$. (No other topology will be used on this set.)

11.5 Definition. (a) Let $G$ be an FHS space, $E_{n}, F_{n} \subset G$ subspaces, and $\lim \inf E_{n}=G$. We say that $F_{n}$ is asymptotically orthogonal to $E_{n}$, if there exists a convergent sequence of admissible norms $\|\cdot\|_{n}$ such that for each $n$, $F_{n}$ is orthogonal to $E_{n}$ w.r.t. $\|\cdot\|_{n}$.

(b) Let $(X, \mathcal{B}, \mathcal{M})$ be a measure class, $\mathcal{E}_{n}, \mathcal{F}_{n} \subset \mathcal{B}$ sub- $\sigma$-fields, and $\lim \inf \mathcal{E}_{n}=\mathcal{B}$. We say that $\mathcal{F}_{n}$ is asymptotically independent of $\mathcal{E}_{n}$, if there exists a convergent sequence of probability measures $\mu_{n} \in \mathcal{M}$ such that for each $n, \mathcal{F}_{n}$ is independent of $\mathcal{E}_{n}$ w.r.t. $\mu_{n}$.

Here lim inf of $\sigma$-fields is their lim inf (according to 11.1) as closed subsets of the space of all measurable sets $(\bmod 0)$ in $(X, \mathcal{B}, \mathcal{M})$. The topology of the space is defined by the distance $\mu(A \triangle B)$ between $A$ and $B$; the metric depends on $\mu \in \mathcal{M}$, but the topology does not.

11.6 Remark. In 11.5(a), 'convergent' means, converging to some admissible norm $\|\cdot\|$. It can be shown that the set of all such norms $\|\cdot\|=\lim _{n}\|\cdot\|_{n}$ is either the empty set, or the whole space of admissible norms. In the latter case $F_{n}$ is asymptotically orthogonal to $E_{n}$, in the former case it is not. Similarly, under the conditions of 11.5(b), the set of all possible measures $\mu=\lim _{n} \mu_{n}$ is either the empty set, or the whole $\mathcal{M}$. However, we do not need it.

${ }^{33}$ You can probably write a simpler equivalent metric. 
11.7 Theorem. Let $G$ be an FHS space, $E_{n}, F_{n} \subset G$ subspaces ( $n=$ $1,2, \ldots)$ such that $\liminf E_{n}=G, \limsup F_{n}=\{0\}$, and $G=E_{n} \oplus F_{n}$ (in the FHS sense) for all $n$. Then there exist subspaces $C_{n}, D_{n} \subset G$ such that $E_{n}=C_{n} \oplus D_{n}$ (in the FHS sense), $\liminf C_{n}=G$, and $D_{n} \oplus F_{n}$ is asymptotically orthogonal to $C_{n}$.

The proof is postponed to the next section.

If $(X, \mathcal{B}, \mathcal{M})$ is a measure class and sub- $\sigma$-fields $\mathcal{E}, \mathcal{F} \subset \mathcal{B}$ satisfy $\mathcal{B}=$ $\mathcal{E} \otimes \mathcal{F}$, then $(X, \mathcal{B}, \mathcal{M})$ decomposes into the product of two measure classes. These may be thought of as quotient spaces $(X, \mathcal{B}, \mathcal{M}) / \mathcal{E}$ and $(X, \mathcal{B}, \mathcal{M}) / \mathcal{F}$, or as $\left(X, \mathcal{E},\left.\mathcal{M}\right|_{\mathcal{E}}\right)$ and $\left(X, \mathcal{F},\left.\mathcal{M}\right|_{\mathcal{F}}\right)$ where $\left.\mathcal{M}\right|_{\mathcal{E}}$ is the equivalence class of measures on $(X, \mathcal{E})$ containing restrictions $\left.\mu\right|_{\mathcal{E}}$ of measures $\mu \in \mathcal{M}$. Some $\mu \in \mathcal{M}$ are product measures satisfying the equality $\mu=\left(\left.\mu\right|_{\mathcal{E}}\right) \otimes\left(\left.\mu\right|_{\mathcal{F}}\right)$; others satisfy the equivalence $\mu \sim\left(\left.\mu\right|_{\mathcal{E}}\right) \otimes\left(\left.\mu\right|_{\mathcal{F}}\right)$. Also the Hilbert space decomposes into the tensor product, $L_{2}(X, \mathcal{B}, \mathcal{M})=L_{2}\left(X, \mathcal{E},\left.\mathcal{M}\right|_{\mathcal{E}}\right) \otimes L_{2}\left(X, \mathcal{F},\left.\mathcal{M}\right|_{\mathcal{F}}\right)$. Suppressing all but $\sigma$-fields we may write it shorter as $L_{2}(\mathcal{B})=L_{2}(\mathcal{E}) \otimes L_{2}(\mathcal{F})$. An element of $L_{2}(\mathcal{E})$ may be written as $f \sqrt{\left.\mu\right|_{\mathcal{E}}}$ where $f$ is a $\mathcal{E}$-measurable function on $X$, and $\mu \in \mathcal{M}$.

11.8 Lemma. Let $(X, \mathcal{B}, \mathcal{M})$ be a measure class, $\mathcal{E}_{n}, \mathcal{F}_{n} \subset \mathcal{B}$ sub- $\sigma$-fields $(n=1,2, \ldots), \mathcal{B}=\mathcal{E}_{n} \otimes \mathcal{F}_{n}$ for each $n$, lim inf $\mathcal{E}_{n}=\mathcal{B}$, and $\mathcal{F}_{n}$ asymptotically independent of $\mathcal{E}_{n}$. Then there exists a probability measure $\mu \in \mathcal{M}$ such that

$$
\liminf _{n \rightarrow \infty}\left(L_{2}\left(\mathcal{E}_{n}\right) \otimes \sqrt{\left.\mu\right|_{\mathcal{F}_{n}}}\right)=L_{2}(\mathcal{B}) .
$$

That is, for every $\psi \in L_{2}(\mathcal{B})$ there exist $\xi_{n} \in L_{2}\left(\mathcal{E}_{n}\right)$ such that $\| \psi-\xi_{n} \otimes$ $\sqrt{\left.\mu\right|_{\mathcal{F}_{n}}} \| \rightarrow 0$ when $n \rightarrow \infty$.

Proof. We take probability measures $\mu, \mu_{n} \in \mathcal{M}$ such that $\mathcal{E}_{n}, \mathcal{F}_{n}$ are $\mu_{n^{-}}$ independent and $\mu_{n} \rightarrow \mu$. We consider an arbitrary finite set $K \subset \mathbb{R}$, an arbitrary $\mathcal{B}$-measurable function $f: X \rightarrow K$, and the corresponding vector $\psi=f \sqrt{\mu} \in L_{2}(X, \mathcal{B}, \mathcal{M})$. Using the fact that $\liminf \mathcal{E}_{n}=\mathcal{B}$, we construct $\mathcal{E}_{n}$-measurable functions $f_{n}: X \rightarrow K$ such that $f_{n} \rightarrow f$ in measure; then $\left\|\left(f-f_{n}\right) \sqrt{\mu}\right\| \rightarrow 0$. Independence of $\mathcal{E}_{n}, \mathcal{F}_{n}$ w.r.t. $\mu_{n}$ means that $\sqrt{\mu_{n}}=$ $\sqrt{\mu_{n} \mid \mathcal{E}_{n}} \otimes \sqrt{\mu_{n} \mid \mathcal{F}_{n}}$. Taking $\xi_{n}=f_{n} \sqrt{\mu_{n} \mid \mathcal{E}_{n}} \in L_{2}\left(\mathcal{E}_{n}\right)$ we get

$$
\begin{aligned}
& \quad\left\|\psi-\xi_{n} \otimes \sqrt{\left.\mu\right|_{\mathcal{F}_{n}}}\right\|=\left\|f \sqrt{\mu}-f_{n} \sqrt{\left.\mu_{n}\right|_{\mathcal{E}_{n}}} \otimes \sqrt{\left.\mu\right|_{\mathcal{F}_{n}}}\right\| \leq \\
& \leq\left\|f \sqrt{\mu}-f_{n} \sqrt{\mu}\right\|+\left\|f_{n} \sqrt{\mu}-f_{n} \sqrt{\mu_{n}}\right\|+\left\|f_{n} \sqrt{\mu_{n}}-f_{n} \sqrt{\mu_{n} \mid \mathcal{E}_{n}} \otimes \sqrt{\left.\mu\right|_{\mathcal{F}_{n}}}\right\| \leq \\
& \leq\left\|\left(f-f_{n}\right) \sqrt{\mu}\right\|+\left\|f_{n}\right\|_{\infty}\left\|\sqrt{\mu}-\sqrt{\mu_{n}}\right\|+\left\|f_{n} \sqrt{\left.\mu_{n}\right|_{\mathcal{E}_{n}}} \otimes\left(\sqrt{\left.\mu_{n}\right|_{\mathcal{F}_{n}}}-\sqrt{\left.\mu\right|_{\mathcal{F}_{n}}}\right)\right\| \rightarrow 0 ;
\end{aligned}
$$

indeed, $\left\|f_{n}\right\|_{\infty}$ is bounded, $\sqrt{\mu_{n}} \rightarrow \sqrt{\mu},\left\|f_{n} \sqrt{\left.\mu_{n}\right|_{\mathcal{E}_{n}}}\right\| \leq\left\|f_{n}\right\|_{\infty}$, and $\| \sqrt{\left.\mu_{n}\right|_{\mathcal{F}_{n}}}-$ $\sqrt{\left.\mu\right|_{\mathcal{F}_{n}}} \| \leq \sqrt{\left\|\left.\mu_{n}\right|_{\mathcal{F}_{n}}-\left.\mu\right|_{\mathcal{F}_{n}}\right\|} \leq \sqrt{\left\|\mu_{n}-\mu\right\|} \rightarrow 0$. It remains to note that such vectors $\psi$ (for all $f$ and $K$ ) are dense in $L_{2}(X, \mathcal{B}, \mathcal{M})$. 
If $E$ is a subspace of an FHS space $G$, then its standard measure class is (canonically isomorphic to) a quotient space of the standard measure class $\left(X_{G}, \mathcal{B}_{G}, \mathcal{M}_{G}\right)$ of $G$; in other words, $\mathcal{B}_{E}$ may be treated as a sub- $\sigma$-field of $\mathcal{B}_{G}$, generated by $\xi_{x}$ for $x \in E$. Thus,

$$
E \subset G \text { implies } \mathcal{B}_{E} \subset \mathcal{B}_{G} \text {. }
$$

If $E_{n} \subset G$ and $\liminf E_{n}=G$, then $\liminf \mathcal{B}_{E_{n}}=\mathcal{B}_{G}$. Indeed, every $\xi_{x}$ for $x \in G$, being equal to $\lim \xi_{x_{n}}, x_{n} \in E_{n}$, is measurable w.r.t. the $\sigma$-field $\lim \inf \mathcal{B}_{E_{n}}$.

If $E_{n}, F_{n} \subset G, \liminf E_{n}=G$, and $F_{n}$ is asymptotically orthogonal to $E_{n}$, then $\mathcal{B}_{F_{n}}$ is asymptotically independent of $\mathcal{B}_{E_{n}}$. Indeed, we have $\|\cdot\|_{n} \rightarrow\|\cdot\|$ and $E_{n} \perp F_{n}$ w.r.t. $\|\cdot\|_{n}$; thus, Gaussian measures $\gamma, \gamma_{n} \in \mathcal{M}$ corresponding to $\|\cdot\|,\|\cdot\|_{n}$ satisfy $\gamma_{n} \rightarrow \gamma$ and $\mathcal{B}_{E_{n}}, \mathcal{B}_{F_{n}}$ are independent w.r.t. $\gamma_{n}$.

Proof of theorem 11.3, part 1: (b) implies (a). Every trace-class operator $R \in$ $\mathcal{B}\left(e^{G}\right)$ satisfying $\operatorname{tr}(R)=0$ is an (infinite) linear combination of two-dimensional operators $R=\left|\psi_{1}\right\rangle\left\langle\psi_{1}|-| \psi_{2}\right\rangle\left\langle\psi_{2}\right|, \psi_{1}, \psi_{2} \in e^{G},\left\|\psi_{1}\right\|=\left\|\psi_{2}\right\|=1$. It is enough to prove that

$$
\sup _{A \in \mathcal{A}_{n},\|A\| \leq 1}\left|\left\langle A \psi_{1}, \psi_{1}\right\rangle-\left\langle A \psi_{2}, \psi_{2}\right\rangle\right| \rightarrow 0 \quad \text { for } n \rightarrow \infty .
$$

Theorem 11.7 (to be proven later) gives us subspaces $C_{n}, D_{n} \subset G$ such that $E_{n}=C_{n} \oplus D_{n}$ (in the FHS sense), $\liminf C_{n}=G$, and $D_{n} \oplus F_{n}$ is asymptotically orthogonal to $C_{n}$. The corresponding sub- $\sigma$-fields satisfy $\liminf \mathcal{B}_{C_{n}}=\mathcal{B}_{G}$, and $\mathcal{B}_{D_{n} \oplus F_{n}}$ is asymptotically independent of $\mathcal{B}_{C_{n}}$; also, $\mathcal{B}_{C_{n}} \otimes \mathcal{B}_{D_{n} \oplus F_{n}}=\mathcal{B}_{G}$ (since $C_{n} \oplus D_{n} \oplus F_{n}=G$ in the FHS sense). Lemma 11.8 gives us a representation

$$
\psi=\lim _{n \rightarrow \infty}\left(\xi_{n} \otimes \chi_{n}\right)
$$

for an arbitrary unit vector $\psi \in e^{G}=L_{2}\left(\mathcal{B}_{G}\right)$; here $\xi_{n} \in e^{C_{n}}=L_{2}\left(\mathcal{B}_{C_{n}}\right)$, $\left\|\xi_{n}\right\|=1, \chi_{n} \in e^{D_{n} \oplus F_{n}}=L_{2}\left(\mathcal{B}_{D_{n} \oplus F_{n}}\right),\left\|\chi_{n}\right\|=1$, and these $\chi_{n}$ (unlike $\xi_{n}$ ) do not depend on $\psi$.

Let $A \in \mathcal{A}_{n}=\mathbf{1} \otimes \mathcal{B}\left(e^{F_{n}}\right) \subset \mathcal{B}\left(e^{G}\right),\|A\| \leq 1$. We have $e^{G}=e^{C_{n}} \otimes e^{D_{n}} \otimes$ $e^{F_{n}}=e^{C_{n}} \otimes e^{D_{n} \oplus F_{n}}, A \in \mathbf{1} \otimes \mathcal{B}\left(e^{D_{n} \oplus F_{n}}\right) ; A=\mathbf{1} \otimes \tilde{A}, \tilde{A} \in \mathcal{B}\left(e^{D_{n} \oplus F_{n}}\right),\|\tilde{A}\| \leq 1$. Thus, $\left\langle A\left(\xi_{n} \otimes \chi_{n}\right), \xi_{n} \otimes \chi_{n}\right\rangle=\left\langle\tilde{A} \chi_{n}, \chi_{n}\right\rangle$, which does not depend on $\psi$. It remains to note that $\left|\langle A \psi, \psi\rangle-\left\langle A\left(\xi_{n} \otimes \chi_{n}\right), \xi_{n} \otimes \chi_{n}\right\rangle\right| \leq\|A\| \cdot \||\psi\rangle\left\langle\psi|-| \xi_{n} \otimes\right.$ $\left.\chi_{n}\right\rangle\left\langle\xi_{n} \otimes \chi_{n}\right|\|\leq 2\| \psi-\xi_{n} \otimes \chi_{n} \| \rightarrow 0$.

\footnotetext{
${ }^{34}$ As usual, $|\psi\rangle\langle\psi|$ stands for the one-dimensional projection $x \mapsto\langle x, \psi\rangle \psi$.
} 
The first part is proven for the real case, but the second part will be proven for the complex case (that is, complex Hilbert spaces out of real FHS spaces); as was said, the two cases are equivalent.

Let $G=E \oplus F$ be the direct sum of two FHS spaces, $\|\cdot\|$ an admissible norm on $G$ (note that $E, F$ need not be orthogonal w.r.t. $\|\cdot\|$ ), and $\gamma \in \mathcal{M}_{G}$ the Gaussian measure on $X_{G}$ corresponding to $\|\cdot\|$. Recall operators $U_{x}, V_{y}$ $\left(x \in G, y \in G^{\prime}\right)$ on the Hilbert space $e_{\mathbb{C}}^{G}$ and their properties stated in 8.11(c). We treat $E, F$ as subspaces of $G$, and their duals $E^{\prime}, F^{\prime}$ as subspaces of $G^{\prime}$ (according to $G=E \oplus F$, irrespective of $\|\cdot\|$ ). In the algebra $\mathcal{B}\left(e_{\mathbb{C}}^{G}\right)=$ $\mathcal{B}\left(e_{\mathbb{C}}^{E} \otimes e_{\mathbb{C}}^{F}\right)$ we introduce a factor $\mathcal{A}=\mathbf{1} \otimes \mathcal{B}\left(e_{\mathbb{C}}^{F}\right)$. (Note that the vector $\sqrt{\gamma} \in e_{\mathbb{C}}^{E} \otimes e_{\mathbb{C}}^{F}$ need not be a product vector.)

11.9 Lemma. There exists an increasing function $M:[0, \infty) \rightarrow[0, \infty)$ such that $M(r)>0$ for all $r>0$, and for all $G, E, F, \mathcal{A},\|\cdot\|, \gamma$ as above,

$$
\begin{gathered}
\sup _{A \in \mathcal{A},\|A\| \leq 1}\left|\langle A \sqrt{\gamma}, \sqrt{\gamma}\rangle-\left\langle A V_{y} \sqrt{\gamma}, V_{y} \sqrt{\gamma}\right\rangle\right| \geq M\left(\operatorname{dist}\left(y, E^{\prime}\right)\right), \\
\sup _{A \in \mathcal{A},\|A\| \leq 1}\left|\langle A \sqrt{\gamma}, \sqrt{\gamma}\rangle-\left\langle A U_{x} \sqrt{\gamma}, U_{x} \sqrt{\gamma}\right\rangle\right| \geq M(\operatorname{dist}(x, E))
\end{gathered}
$$

for all $x \in G, y \in G^{\prime}$; here the distance $\operatorname{dist}(x, E)=\inf _{z \in E}\|x-z\|$ is taken w.r.t. the norm $\|\cdot\|$, and $\operatorname{dist}\left(y, E^{\prime}\right)$ - w.r.t. the dual norm on $G^{\prime}$.

Proof. The operator $A=U_{x}$ for $x \in F$ belongs to $\mathcal{A}$, and $\|A\| \leq 1$. We have

$$
\begin{gathered}
\langle A \sqrt{\gamma}, \sqrt{\gamma}\rangle=\exp \left(-\frac{1}{2}\|x\|^{2}\right) ; \\
\left\langle A V_{y} \sqrt{\gamma}, V_{y} \sqrt{\gamma}\right\rangle=\left\langle U_{x} V_{y} \sqrt{\gamma}, V_{y} \sqrt{\gamma}\right\rangle=e^{-i\langle x, y\rangle}\left\langle V_{y} U_{x} \sqrt{\gamma}, V_{y} \sqrt{\gamma}\right\rangle= \\
=e^{-i\langle x, y\rangle}\left\langle U_{x} \sqrt{\gamma}, \sqrt{\gamma}\right\rangle=\exp \left(-i\langle x, y\rangle-\frac{1}{2}\|x\|^{2}\right) ; \\
\left|\langle A \sqrt{\gamma}, \sqrt{\gamma}\rangle-\left\langle A V_{y} \sqrt{\gamma}, V_{y} \sqrt{\gamma}\right\rangle\right|=\left|1-e^{-i\langle x, y\rangle}\right| \exp \left(-\frac{1}{2}\|x\|^{2}\right)= \\
=2\left|\sin \frac{\langle x, y\rangle}{2}\right| \exp \left(-\frac{1}{2}\|x\|^{2}\right) .
\end{gathered}
$$

Taking into account that $\operatorname{dist}\left(y, E^{\prime}\right)=\sup _{x \in F,\|x\| \leq 1}|\langle x, y\rangle|$ we get the first inequality for

$$
M(r)=\sup _{u \geq 0} 2 \sin \left(\frac{1}{2} r u\right) \exp \left(-\frac{1}{2} u^{2}\right)=\sup _{\varphi \geq 0}\left(\exp \left(-\frac{\varphi^{2}}{2 r^{2}}\right) \cdot 2\left|\sin \frac{\varphi}{2}\right|\right) .
$$


The proof of the second inequality is quite similar. Only $U_{x}, V_{y}$ are interchanged, and $\exp \left(-\frac{1}{8}\|y\|^{2}\right)$ appears instead of $\exp \left(-\frac{1}{2}\|x\|^{2}\right)$, which leads to $M(2 r)$ instead of $M(r)$.

Proof of theorem 11.3, part 2: (a) implies (b). We choose a Gaussian measure $\gamma \in \mathcal{M}_{G}$ and apply (a) to the operator $R=|\sqrt{\gamma}\rangle\left\langle\sqrt{\gamma}|-| V_{y} \sqrt{\gamma}\right\rangle\left\langle V_{y} \sqrt{\gamma}\right|$ for an arbitrary $y \in G^{\prime}$, and also to $R=|\sqrt{\gamma}\rangle\left\langle\sqrt{\gamma}|-| U_{x} \sqrt{\gamma}\right\rangle\left\langle U_{x} \sqrt{\gamma}\right|$ for an arbitrary $x \in G$ :

$$
\begin{gathered}
\sup _{A \in \mathcal{A},\|A\| \leq 1}\left|\langle A \sqrt{\gamma}, \sqrt{\gamma}\rangle-\left\langle A V_{y} \sqrt{\gamma}, V_{y} \sqrt{\gamma}\right\rangle\right| \rightarrow 0, \\
\sup _{A \in \mathcal{A},\|A\| \leq 1}\left|\langle A \sqrt{\gamma}, \sqrt{\gamma}\rangle-\left\langle A U_{x} \sqrt{\gamma}, U_{x} \sqrt{\gamma}\right\rangle\right| \rightarrow 0
\end{gathered}
$$

for $n \rightarrow 0$. Lemma 11.9 gives

$$
\operatorname{dist}\left(y, E_{n}^{\prime}\right) \rightarrow 0, \quad \operatorname{dist}\left(x, E_{n}\right) \rightarrow 0 .
$$

The latter shows that $x \in \lim \inf E_{n}$, which means that $\lim \inf E_{n}=G$. The former shows that

$$
\sup _{z \in F_{n},\|z\| \leq 1}|\langle y, z\rangle| \rightarrow 0 \quad \text { for } n \rightarrow 0
$$

which means that $\lim \sup F_{n}=\{0\}$.

So, Theorem 11.3 follows from Theorem 11.7 .

\section{Ensuring asymptotic orthogonality}

The sole goal of this section is to prove Theorem 11.7 .

12.1 Lemma. Let $G$ be an FHS space, and $F_{n} \subset G$ subspaces. Then the following conditions are equivalent.

(a) $\lim \sup F_{n}=\{0\}$;

(b) for every finite-dimensional subspaces $E_{1}, E_{2}, \ldots$ such that $\lim \inf E_{n}=$ $G$ there exist integers $k_{1} \leq k_{2} \leq \ldots$ such that $k_{n} \rightarrow \infty$ and $F_{n}$ is asymptotically orthogonal to $E_{k_{n}}$.

Proof. The implication "(b) $\Rightarrow$ (a)" is simpler, and will not be used; I leave it to the reader. Assume (a). We choose an admissible norm on $G$, thus turning $G$ into a Hilbert space. Condition (a) gives

$$
\forall x \in G \quad \sup _{f \in F_{n},\|f\| \leq 1}\langle f, x\rangle \underset{n \rightarrow \infty}{\longrightarrow} 0
$$


that is,

$$
\forall x \in G \quad \angle\left(x, F_{n}\right) \underset{n \rightarrow \infty}{\longrightarrow} \frac{\pi}{2},
$$

where the angle is defined by $\cos \angle\left(x, F_{n}\right)=\sup \left\{\langle x, f\rangle: f \in F_{n},\|f\| \leq 1\right\}$. Therefore

$$
\forall E \quad \angle\left(E, F_{n}\right) \underset{n \rightarrow \infty}{\longrightarrow} \frac{\pi}{2}
$$

where $E$ runs over finite-dimensional subspaces, and $\cos \angle\left(E, F_{n}\right)=\sup \{\langle e, f\rangle$ : $\left.e \in E, f \in F_{n},\|e\| \leq 1,\|f\| \leq 1\right\}$. The following lemma completes the proof, provided that $k_{n}$ tends to $\infty$ slowly enough. Namely, in terms of $\delta(\cdot, \cdot)$ introduced there, it suffices that $\delta\left(\angle\left(E_{k_{n}}, F_{n}\right), \operatorname{dim} E_{k_{n}}\right) \underset{n \rightarrow \infty}{\longrightarrow} 0$.

12.2 Lemma. Let $G$ be an FHS-space, $E, F \subset G$ subspaces, $\operatorname{dim}(E)<\infty$, $E \cap F=\{0\}$. Then for every admissible norm $\|\cdot\|_{1}$ there exists an admissible norm $\|\cdot\|_{2}$ such that $E, F$ are orthogonal w.r.t. $\|\cdot\|_{2}$, and

$$
\operatorname{dist}\left(\|\cdot\|_{1},\|\cdot\|_{2}\right) \leq \delta(\angle(E, F), \operatorname{dim} E)
$$

for some function $\delta:\left[0, \frac{\pi}{2}\right] \times\{0,1,2, \ldots\} \rightarrow(0, \infty)$ such that for every $n$ $\delta(\alpha, n) \rightarrow 0$ for $\alpha \rightarrow \frac{\pi}{2}$. 阿

Proof. We equip $G$ with the norm $\|\cdot\|_{1}$, thus turning $G$ into a Hilbert space, and consider orthogonal projections $Q_{E}, Q_{F}$ onto $E, F$ respectively. Introduce subspaces $E \cap F^{\perp}, E^{\perp} \cap F, E^{\perp} \cap F^{\perp}$ (here $E^{\perp}$ is the orthogonal complement of $E$ ); the subspaces are orthogonal to each other, and invariant under both $Q_{E}$ and $Q_{F}$. Therefore

$$
G=G_{0} \oplus\left(E \cap F^{\perp}\right) \oplus\left(E^{\perp} \cap F\right) \oplus\left(E^{\perp} \cap F^{\perp}\right), \quad \text { (orthogonal sum) }
$$

where $G_{0}$ is another subspace invariant under $Q_{E}, Q_{F}$ (since these operators are Hermitian). Introduce $E_{0}=E \cap G_{0}, F_{0}=F \cap G_{0}$, then $Q_{E} h_{0}=Q_{E_{0}} h_{0}$ for all $h_{0} \in G_{0}$ (since $Q_{E}$ commutes with $Q_{G_{0}}$ ), and $Q_{F} h_{0}=Q_{F_{0}} h_{0}$. We may get rid of $G_{0}^{\perp}$ by letting

$$
\left\|h_{0}+h_{1}\right\|_{2}^{2}=\left\|h_{0}\right\|_{2}^{2}+\left\|h_{1}\right\|_{1}^{2} \text { for all } h_{0} \in G_{0}, h_{1} \in G_{0}^{\perp} .
$$

In other words, we'll construct $\|\cdot\|_{2}$ on $G_{0}$ while preserving both the given norm on $G_{0}^{\perp}$ and the orthogonality of $G_{0}, G_{0}^{\perp}$. Now we forget about $G_{0}^{\perp}$, assuming that $G=G_{0}, E=E_{0}, F=F_{0}$.

So, we have $E \cap F=\{0\}, E \cap F^{\perp}=\{0\}, E^{\perp} \cap F=\{0\}, E^{\perp} \cap F^{\perp}=\{0\}$. The latter implies $\operatorname{dim}\left(F^{\perp}\right) \leq \operatorname{codim}\left(E^{\perp}\right)=\operatorname{dim} E$. Similarly, $\operatorname{dim} F \leq$ $\operatorname{dim} E$. Therefore $G$ is finite-dimensional, $\operatorname{dim} G \leq 2 \operatorname{dim} E$.

\footnotetext{
${ }^{35}$ Of course, $\delta$ does not depend on $G, E, F$.
} 
Both $Q_{E}$ and $Q_{F}$ commute with the Hermitian operator $C=\frac{1}{2}\left(2 Q_{E}-\right.$ $1)\left(2 Q_{F}-1\right)+\frac{1}{2}\left(2 Q_{F}-1\right)\left(2 Q_{E}-1\right)$. The spectrum of $C$ consists of some numbers $\cos 2 \varphi_{k}$ of multiplicity 2 (though, some $\varphi_{k}$ may coincide), and $0<$ $\varphi_{k}<\frac{\pi}{2}$ (the case $\varphi_{k}=0$ is excluded by $E \cap F=\{0\}$; the case $\varphi_{k}=\pi / 2$ is excluded by $\left.E \cap F^{\perp}=\{0\}, E^{\perp} \cap F=\{0\}, E^{\perp} \cap F^{\perp}=\{0\}\right)$. Accordingly, $G$ decomposes into the (orthogonal) direct sum of planes, $G=G_{1} \oplus \cdots \oplus G_{d}$, $\operatorname{dim} G_{k}=2$, invariant under $Q_{E}, Q_{F}$. Subspaces $E_{k}=E \cap G_{k}, F_{k}=F \cap G_{k}$ are two lines on the plane $G_{k}$, and $\angle\left(E_{k}, F_{k}\right)=\varphi_{k} ; k=1, \ldots, d ; d \leq \operatorname{dim} E$. Clearly,

$$
\angle(E, F)=\min \left(\varphi_{1}, \ldots, \varphi_{d}\right) .
$$

We construct $\|\cdot\|_{2}$ on each $G_{k}$ separately, while preserving their orthogonality. Elementary 2-dimensional geometry shows that the corresponding numbers $\lambda_{k}^{\prime}, \lambda_{k}^{\prime \prime}$ (mentioned in (11.4); two numbers for each plane) are, in the optimal case,

$$
\lambda_{k}^{\prime}=\left(\tan \frac{\varphi_{k}}{2}\right)^{-1 / 2}, \quad \lambda_{k}^{\prime \prime}=\left(\tan \frac{\varphi_{k}}{2}\right)^{1 / 2} .
$$

The corresponding right-hand side of (11.4) is

$$
\cos \operatorname{dist}\left(\|\cdot\|_{1},\|\cdot\|_{2}\right)=\prod_{k=1}^{d}\left(\frac{\tan ^{-1 / 4} \frac{\varphi_{k}}{2}+\tan ^{1 / 4} \frac{\varphi_{k}}{2}}{2}\right)^{-1},
$$

which gives the needed result for

$$
\delta(\alpha, n)=\arccos \left(\frac{\tan ^{-1 / 4} \frac{\alpha}{2}+\tan ^{1 / 4} \frac{\alpha}{2}}{2}\right)^{-n}
$$

Proof of theorem 11.7. We choose an admissible norm on $G$, thus turning $G$ into a Hilbert space over $\mathbb{R}$. Let $L \subset G$ be a finite-dimensional subspace, $L \neq\{0\}$. For any given $n$ consider the pair $L, E_{n}$. Its geometry may be described (similarly to the proof of Lemma 12.2) via angles $\varphi_{1}^{(n)}, \ldots, \varphi_{d_{n}}^{(n)} \in$ $\left[0, \frac{\pi}{2}\right), d_{n} \leq \operatorname{dim} L$. This time, zero angles are allowed, since $L \cap E_{n}$ need not be $\{0\}$. It may happen that $d_{n}<\operatorname{dim} L$, since $L \cap E_{n}^{\perp}$ need not be $\{0\}$. However,

$$
\sup _{x \in L, x \neq 0} \angle\left(x, E_{n}\right)=\alpha_{n} \rightarrow 0 \text { for } n \rightarrow \infty,
$$

since liminf $E_{n}=G$; for large $n$ we have $\alpha_{n}<\pi / 2$ which implies $d_{n}=d=$ $\operatorname{dim} L$ and $\max \left(\varphi_{1}^{(n)}, \ldots, \varphi_{d}^{(n)}\right)=\alpha_{n}$. We may send $L$ into $E_{n}$ rotating it by $\varphi_{1}^{(n)}, \ldots, \varphi_{d}^{(n)}$. In other words, there is a rotation $U_{n}: G \rightarrow G$ such that

$$
U_{n}(L) \subset E_{n} \quad \text { and } \quad\left\|U_{n}-1\right\| \leq 2 \sin \frac{\alpha_{n}}{2} \underset{n \rightarrow \infty}{\longrightarrow} 0
$$


We choose subspaces $L_{k} \subset G$ such that $\operatorname{dim} L_{k}=k$ and $\lim \inf L_{k}=G$. Introduce

$$
\alpha_{k, n}=\sup _{x \in L_{k}, x \neq 0} \angle\left(x, E_{n}\right)
$$

then $\alpha_{k, n} \underset{n \rightarrow \infty}{\longrightarrow} 0$ for each $k$. On the other hand, introduce

$$
\beta_{k, n}=\frac{\pi}{2}-\angle\left(L_{k}, F_{n}\right)
$$

Similarly to the proof of Lemma 12.1 we have $\beta_{k, n} \underset{n \rightarrow \infty}{\longrightarrow} 0$ for each $k$; therefore $\mathrm{j}^{37} \delta\left(\frac{\pi}{2}-\beta_{k_{n}, n}, k_{n}\right) \underset{n \rightarrow \infty}{\longrightarrow} 0$ if $k_{n}$ tends to $\infty$ slowly enough. However, we choose $k_{1} \leq k_{2} \leq \cdots, k_{n} \rightarrow \infty$ so as to satisfy a stronger condition:

$$
\delta\left(\frac{\pi}{2}-\alpha_{k_{n}, n}-\beta_{k_{n}, n}, k_{n}\right) \underset{n \rightarrow \infty}{\longrightarrow} 0 \text {. }
$$

We take

$$
C_{n}=U_{n}\left(L_{k_{n}}\right),
$$

where rotations $U_{n}$ satisfy $U_{n}\left(L_{k_{n}}\right) \subset E_{n}$ and $\left\|U_{n}-1\right\| \leq 2 \sin \left(\frac{1}{2} \alpha_{k_{n}, n}\right) \rightarrow 0$. Then $\liminf C_{n}=G$, and

$$
\frac{\pi}{2}-\angle\left(C_{n}, F_{n}\right) \leq \alpha_{k_{n}, n}+\beta_{k_{n}, n}
$$

due to Lemma 12.2, there exist admissible norms $\|\cdot\|_{n} \rightarrow\|\cdot\|$ such that $F_{n}$ is orthogonal to $C_{n}$ w.r.t. $\|\cdot\|_{n}$. Consider the orthogonal complement $M_{n}$ of $C_{n}$ w.r.t. $\|\cdot\|_{n}$; clearly, $M_{n}$ is asymptotically orthogonal to $C_{n}$. We have $F_{n} \subset M_{n}$ and $G=C_{n} \oplus M_{n}$ (in the FHS-sense). On the other hand, $C_{n} \subset E_{n}$ and $G=E_{n} \oplus F_{n}$. Applying Remark 9.1 (for each $n$ separately) to $G_{1}=C_{n}$, $G_{1,2}=E_{n}, G_{2,3}=M_{n}, G_{3}=F_{n}$ we see that the subspace

$$
D_{n}=E_{n} \cap M_{n}
$$

satisfies $C_{n} \oplus D_{n} \oplus F_{n}=G$ and $C_{n} \oplus D_{n}=E_{n}$ (and also $D_{n} \oplus F_{n}=M_{n}$ ). Asymptotical orthogonality of $D_{n} \oplus F_{n}$ to $C_{n}$ follows from their orthogonality w.r.t. $\|\cdot\|_{n}$.

\section{Calculating the invariant}

It is conjectured that there are uncountably many type III $E_{0}$-semigroups which are not cocycle conjugate. (So far we have only one example!)

W. Arveson A99, p. 167]

\footnotetext{
${ }^{36}$ Of course, one may take $L_{1} \subset L_{2} \subset \ldots$

${ }^{37}$ Recall that $\delta(\cdot, \cdot)$ is introduced in Lemma 12.2.
} 
As was said in Sect. 2, we consider sequences $\left(E_{n}\right)$ of elementary sets

$$
E_{n}=\bigcup_{k=0}^{n-1}\left(\frac{1}{n}\left(k+\frac{1-\varepsilon_{n}}{2}\right), \frac{1}{n}\left(k+\frac{1+\varepsilon_{n}}{2}\right)\right)
$$

infinitesimality of $\left(E_{n}\right)$ depends on $\left(\varepsilon_{n}\right)$ and the spectral measure $\nu$ (assumed to satisfy $9.7(\mathrm{~b})$ and (9.14)). Theorem 11.3 states that $\left(E_{n}\right)$ is infinitesimal if and only if

$$
\begin{gathered}
\liminf G_{(0,1) \backslash E_{n}}=G_{(0,1)}, \\
\limsup G_{E_{n}}=\{0\}
\end{gathered}
$$

here $G_{E_{n}}$ is the closure in $L_{2}(\nu)$ of the set of Fourier transforms $\hat{f}$ of functions $f \in L_{2}\left(E_{n}\right)$ (or rather, $f \in L_{2}(\mathbb{R})$ vanishing outside $E_{n}$ ).

13.4 Lemma. If $\varepsilon_{n} \rightarrow 0$ then (13.2) holds.

Proof. For every $f \in L_{2}(0,1)$,

$$
\left\|f-f \cdot \mathbf{1}_{E_{n}}\right\|_{\nu} \leq \mathrm{const} \cdot\left\|f-f \cdot \mathbf{1}_{E_{n}}\right\|_{L_{2}(0,1)} \rightarrow 0 .
$$

13.5 Remark. If $\nu$ satisfies (10.1) then (13.2) holds already for the constant sequence $\varepsilon_{n}=1 / 2$, due to (a slight modification of) Lemma 10.2.

The question is when (13.3) holds. Here is a sufficient condition for arbitrary elementary sets $E_{n} \subset(0,1)$, not just of the form 113.1).

13.6 Lemma. Existence of a function $\beta:(0, \infty) \rightarrow(0, \infty)$ and numbers $\delta_{n} \rightarrow 0$ satisfying conditions (a)-(c) below is sufficient for (13.3):

(a) $\beta$ decreases, but $\lambda \mapsto \lambda^{2} \beta(\lambda)$ increases;

(b) $\nu(d \lambda) \geq \beta(\lambda) d \lambda$;

(c) $\frac{\operatorname{mes}\left(\left(E_{n}\right)_{+\delta_{n}}\right)}{\beta\left(\delta_{n}^{-1}\right)} \rightarrow 0$ for $n \rightarrow \infty$, where $\left(E_{n}\right)_{+\delta_{n}}$ stands for the $\delta_{n^{-}}$ neighborhood of $E_{n}$, and 'mes' for the Lebesgue measure.

Proof. Let $f_{n} \in G_{E_{n}},\left\|f_{n}\right\|_{\nu} \leq 1$. We have to prove that $f_{n} \rightarrow 0$ weakly, that is, $\int \hat{f}_{n}(\lambda) \hat{g}(\lambda) d \lambda \rightarrow 0$ for every $\hat{g}$ such that $\int|\hat{g}(\lambda)|^{2} \frac{d \lambda}{\nu(d \lambda)} d \lambda<\infty$. may restrict ourselves to a dense set of such $\hat{g}$; thus we assume that $\hat{g}$ has a compact support. It follows that $\int|\hat{g}(\lambda)|^{2} d \lambda<\infty$ (since $\beta$ is bounded away

\footnotetext{
${ }^{38}$ For now $\hat{g}$ is not assumed to be a Fourier transform of some $g$, but it will happen soon.
} 
from 0 on the support of $\hat{g}$ ) and $\int|\hat{g}(\lambda)| d \lambda<\infty$. Therefore $\hat{g}$ is the Fourier transform of a function $g \in L_{2}(\mathbb{R}) \cap L_{\infty}(\mathbb{R})$.

For every $\delta \in(0, \infty)$,

$$
\frac{\nu(d \lambda)}{d \lambda} \geq \beta\left(\delta^{-1}\right)\left(\frac{\sin \delta \lambda}{\delta \lambda}\right)^{2} \text { for all } \lambda \in(0, \infty) ;
$$

indeed, for $\lambda \in\left(0, \delta^{-1}\right)$ we have

$$
\left(\frac{\delta \lambda}{\sin \delta \lambda}\right)^{2} \frac{\nu(d \lambda)}{d \lambda} \geq \frac{\nu(d \lambda)}{d \lambda} \geq \beta(\lambda) \geq \beta\left(\delta^{-1}\right)
$$

and for $\lambda \in\left(\delta^{-1}, \infty\right)$ we have

$$
\left(\frac{\delta \lambda}{\sin \delta \lambda}\right)^{2} \frac{\nu(d \lambda)}{d \lambda} \geq(\delta \lambda)^{2} \frac{\nu(d \lambda)}{d \lambda} \geq \delta^{2} \lambda^{2} \beta(\lambda) \geq \delta^{2} \delta^{-2} \beta\left(\delta^{-1}\right) .
$$

We define functions $h_{n}$ by

$$
\hat{h}_{n}(\lambda)=\hat{f}_{n}(\lambda) \frac{\sin \delta_{n} \lambda}{\delta_{n} \lambda}
$$

then $h_{n} \in G_{\left(E_{n}\right)_{+} \delta_{n}}$, since缯

$$
h_{n}(t)=\frac{\text { const }}{2 \delta_{n}} \int_{t-\delta_{n}}^{t+\delta_{n}} f_{n}(s) d s .
$$

We have

$$
\begin{gathered}
\int h_{n}^{2}(t) d t=\int\left|\hat{h}_{n}(\lambda)\right|^{2} d \lambda=\int\left|\hat{f}_{n}(\lambda)\right|^{2}\left(\frac{\sin \delta_{n} \lambda}{\delta_{n} \lambda}\right)^{2} d \lambda \leq \\
\leq \frac{2}{\beta\left(\delta_{n}^{-1}\right)} \int_{0}^{\infty}\left|\hat{f}_{n}(\lambda)\right|^{2} \nu(d \lambda) ; \\
\beta\left(\delta_{n}^{-1}\right)\left\|h_{n}\right\|_{L_{2}}^{2} \leq 2\left\|f_{n}\right\|_{\nu}^{2} \leq 2 ; \quad \sqrt{\beta\left(\delta_{n}^{-1}\right)}\left\|h_{n}\right\|_{L_{2}} \leq \sqrt{2} .
\end{gathered}
$$

However,

$$
\begin{aligned}
\left|\int \hat{h}_{n}(\lambda) \hat{g}(\lambda) d \lambda\right|=\left|\int h_{n}(t) g(t) d t\right| & \\
=\left|\int h_{n}(t) \mathbf{1}_{\left(E_{n}\right)_{+\delta_{n}}}(t) g(t) d t\right| & \leq\left\|h_{n}\right\|\left\|_{L_{2}}\right\| \mathbf{1}_{\left(E_{n}\right)_{+\delta_{n}}} g \|_{L_{2}} \leq \\
\leq & \frac{\sqrt{2}}{\sqrt{\beta\left(\delta_{n}^{-1}\right)}} \sqrt{\operatorname{mes}\left(E_{n}\right)_{+\delta_{n}}}\|g\|_{\infty} \rightarrow 0 .
\end{aligned}
$$

\footnotetext{
${ }^{39} \mathrm{~A}$ constant appears, since we normalize Fourier transform so as to be unitary.
} 
It remains to note that

$$
\begin{gathered}
\left|\int \hat{f}_{n}(\lambda) \hat{g}(\lambda) d \lambda-\int \hat{h}_{n}(\lambda) \hat{g}(\lambda) d \lambda\right| \leq \int\left|\hat{f}_{n}(\lambda)\right|\left(1-\left(\frac{\sin \delta_{n} \lambda}{\delta_{n} \lambda}\right)^{2}\right)|\hat{g}(\lambda)| d \lambda \leq \\
\left(\int\left|\hat{f}_{n}(\lambda)\right|^{2} \nu(d \lambda)\right)^{1 / 2}\left(\int|\hat{g}(\lambda)|^{2} \frac{d \lambda}{\nu(d \lambda)} d \lambda\right)^{1 / 2} \max _{\lambda \in \operatorname{supp} \hat{g}}\left(1-\left(\frac{\sin \delta_{n} \lambda}{\delta_{n} \lambda}\right)^{2}\right) \rightarrow 0 .
\end{gathered}
$$

Now we need a necessary condition for (13.3). Sets $E_{n}$ are again assumed to be of the form (13.1). We introduce functionsty

$$
f_{n}=\frac{1}{\varepsilon_{n}} \mathbf{1}_{E_{n}}, \quad f=\mathbf{1}_{(0,1)} .
$$

If $\left\|f_{n}-f\right\|_{\nu} \rightarrow 0$ then $(13.3)$ is evidently violated. Lemma 10.2 gives us $\left\|f_{n}-f\right\|_{\nu} \rightarrow 0$ for the constant sequence $\varepsilon_{n}=1 / 2$, but now it will be proven for $\varepsilon_{n}$ tending to 0 but not too fast.

Recall that a slowly varying function is a function $\alpha:(0, \infty) \rightarrow(0, \infty)$ such that

$$
\alpha(\lambda)=(1+o(1)) \exp \left(\int_{1}^{\lambda} \frac{\eta\left(\lambda_{1}\right)}{\lambda_{1}} d \lambda_{1}\right) \text { for } \lambda \rightarrow \infty
$$

for some function $\eta(\cdot)$ satisfying $\eta(\lambda) \rightarrow 0$ for $\lambda \rightarrow \infty$; see [Feller, Sect. VIII.9]. Observe that functions (9.12), (9.13) are slowly varying.

13.7 Lemma. Let $\alpha:(0, \infty) \rightarrow(0, \infty)$ be a slowly varying function such that $\nu(d \lambda) \leq \alpha(\lambda) d \lambda$, and $\varepsilon_{n} \rightarrow 0$ satisfy

$$
\frac{1}{\varepsilon_{n}} \alpha\left(\frac{n}{\varepsilon_{n}}\right) \rightarrow 0 \quad \text { for } n \rightarrow \infty .
$$

Then $\left\|f_{n}-f\right\|_{\nu} \rightarrow 0$ for $n \rightarrow \infty$.

Proof. An elementary calculation gives

$$
\left|\hat{f}_{n}(\lambda)\right|=\frac{\text { const }}{\varepsilon_{n}}\left|\frac{\sin \frac{\lambda \varepsilon_{n}}{n} \sin \frac{\lambda}{2}}{\lambda \sin \frac{\lambda}{2 n}}\right| .
$$

\footnotetext{
${ }^{40}$ Here $\mathbf{1}_{E}(t)=1$ for $t \in E$, otherwise 0 .
} 
We have to prove that $\int\left|\hat{f}_{n}(\lambda)-\hat{f}(\lambda)\right|^{2} \nu(d \lambda) \rightarrow 0$. First of all, $\hat{f}_{n}(\lambda) \rightarrow \hat{f}(\lambda)$ uniformly on $\lambda \in(0, M)$ for every $M<\infty$. Therefore it is enough to prove that $\int_{M}^{\infty}\left|\hat{f}_{n}(\lambda)\right|^{2} \nu(d \lambda) \rightarrow 0$ for $M \rightarrow \infty$ uniformly in $n$. We have

$$
\begin{aligned}
\int_{M}^{\infty}\left|\hat{f}_{n}(\lambda)\right|^{2} \alpha(\lambda) d \lambda \leq \frac{\text { const }}{\varepsilon_{n}^{2}} \int_{M}^{\infty} \alpha(\lambda) \frac{\sin ^{2} \frac{\lambda \varepsilon_{n}}{n}}{\lambda^{2}} \frac{\sin ^{2} \frac{\lambda}{2}}{\sin ^{2} \frac{\lambda}{2 n}} d \lambda= \\
=\frac{\text { const }}{\varepsilon_{n}^{2}}\left(\int_{M}^{\pi n} \ldots d \lambda+\sum_{k=1}^{\infty} \int_{(2 k-1) \pi n}^{(2 k+1) \pi n} \ldots d \lambda\right) .
\end{aligned}
$$

The function $\frac{\sin ^{2} \frac{\lambda}{2}}{\sin ^{2} \frac{\lambda}{2 n}}=\left|\sum_{k=0}^{n-1} \exp (i k \lambda / n)\right|^{2}$ has period $2 \pi n$, and its mean value (over the period) is equal to $n$. On the other hand, $\frac{1}{\lambda^{2}} \sin ^{2} \frac{\lambda \varepsilon_{n}}{n} \leq$ $\min \left(\frac{\varepsilon_{n}^{2}}{n^{2}}, \frac{1}{\lambda^{2}}\right)$. Thus, for $n \rightarrow \infty$,

$$
\begin{aligned}
& \sum_{k=1}^{\infty} \int_{(2 k-1) \pi n}^{(2 k+1) \pi n} \alpha(\lambda) \frac{\sin ^{2} \frac{\lambda \varepsilon_{n}}{n}}{\lambda^{2}} \frac{\sin ^{2} \frac{\lambda}{2}}{\sin ^{2} \frac{\lambda}{2 n}} d \lambda \leq \\
& \leq \sum_{k=1}^{\infty}\left(\max _{\lambda \in[(2 k-1) \pi n,(2 k+1) \pi n]} \alpha(\lambda)\right) \min \left(\frac{\varepsilon_{n}^{2}}{n^{2}}, \frac{1}{(2 k-1)^{2} \pi^{2} n^{2}}\right) \cdot 2 \pi n \cdot n \leq \\
& \leq(1+o(1)) n \int_{\pi n}^{\infty} \alpha(\lambda) \min \left(\frac{\varepsilon_{n}^{2}}{n^{2}}, \frac{1}{\lambda^{2}}\right) d \lambda= \\
& \quad=(1+o(1)) n\left(\frac{\varepsilon_{n}^{2}}{n^{2}} \int_{\pi n}^{n / \varepsilon_{n}} \alpha(\lambda) d \lambda+\int_{n / \varepsilon_{n}}^{\infty} \alpha(\lambda) \lambda^{-2} d \lambda\right) .
\end{aligned}
$$

However, $\int_{\pi n}^{n / \varepsilon_{n}} \alpha(\lambda) d \lambda=(1+o(1)) \frac{n}{\varepsilon_{n}} \alpha\left(\frac{n}{\varepsilon_{n}}\right)$; see [Feller, Th. 1 of Sect. VIII.9]. Similarly, $\int_{n / \varepsilon_{n}}^{\infty} \alpha(\lambda) \lambda^{-2} d \lambda=(1+o(1)) \alpha\left(\frac{n}{\varepsilon_{n}}\right) \int_{n / \varepsilon_{n}}^{\infty} \lambda^{-2} d \lambda=(1+o(1)) \frac{\varepsilon_{n}}{n} \alpha\left(\frac{n}{\varepsilon_{n}}\right)$. So,

$$
\frac{1}{\varepsilon_{n}^{2}} \sum_{k=1}^{\infty} \int_{(2 k-1) \pi n}^{(2 k+1) \pi n} \ldots d \lambda \leq(1+o(1)) \frac{n}{\varepsilon_{n}^{2}} \cdot 2 \frac{\varepsilon_{n}}{n} \alpha\left(\frac{n}{\varepsilon_{n}}\right)=(1+o(1)) \frac{2}{\varepsilon_{n}} \alpha\left(\frac{n}{\varepsilon_{n}}\right) \rightarrow 0 .
$$

It remains to prove that

$$
\frac{1}{\varepsilon_{n}^{2}} \int_{M}^{\pi n} \alpha(\lambda) \frac{\sin ^{2} \frac{\lambda \varepsilon_{n}}{n}}{\lambda^{2}} \frac{\sin ^{2} \frac{\lambda}{2}}{\sin ^{2} \frac{\lambda}{2 n}} d \lambda \rightarrow 0 \quad \text { for } M \rightarrow \infty
$$

uniformly in $n(n \geq M / \pi)$. We have $\sin \frac{\lambda}{2 n} \geq \frac{2}{\pi} \frac{\lambda}{2 n}, \sin ^{2} \frac{\lambda}{2} \leq 1, \sin ^{2} \frac{\lambda \varepsilon_{n}}{n} \leq$ 
$\left(\frac{\lambda \varepsilon_{n}}{n}\right)^{2}, \alpha(\lambda) \leq\|\alpha\|_{\infty} ;$ thus

$$
\begin{aligned}
& \frac{1}{\varepsilon_{n}^{2}} \int_{M}^{\pi n} \ldots d \lambda \leq \frac{1}{\varepsilon_{n}^{2}}\|\alpha\|_{\infty}\left(\frac{\pi}{2}\right)^{2} \int_{M}^{\infty} \frac{\left(\frac{\lambda \varepsilon_{n}}{n}\right)^{2}}{\lambda^{2}\left(\frac{\lambda}{2 n}\right)^{2}} d \lambda= \\
& \quad=\frac{1}{\varepsilon_{n}^{2}}\|\alpha\|_{\infty}\left(\frac{\pi}{2}\right)^{2}\left(2 \varepsilon_{n}\right)^{2} \int_{M}^{\infty} \frac{d \lambda}{\lambda^{2}}=\pi^{2}\|\alpha\|_{\infty} \frac{1}{M}
\end{aligned}
$$

Having a sufficient condition (given by Lemma 13.6) and a necessary condition (given by Lemma 13.7), we want to compare them. If $E_{n}$ in Lemma 13.6 is of the form (13.1) then $\operatorname{mes}\left(\left(E_{n}\right)_{+\delta_{n}}\right)=\varepsilon_{n}+2 n \delta_{n}$ (unless it exceeds 1 ), and 13.6(c) becomes

$$
\frac{\varepsilon_{n}+2 n \delta_{n}}{\beta\left(\delta_{n}^{-1}\right)} \rightarrow 0 \quad \text { for some }\left(\delta_{n}\right)_{n=1}^{\infty} .
$$

If we $\operatorname{try} \delta_{n}=\varepsilon_{n} / n$, the condition becomes $\varepsilon_{n} / \beta\left(n / \varepsilon_{n}\right) \rightarrow 0$, that is,

$$
\frac{1}{\varepsilon_{n}} \beta\left(\frac{n}{\varepsilon_{n}}\right) \rightarrow \infty
$$

which implies the necessary condition, $\left(1 / \varepsilon_{n}\right) \alpha\left(n / \varepsilon_{n}\right) \not \rightarrow 0$, provided that $\alpha(\cdot) \geq \beta(\cdot)$.

Moreover, (13.9) is equivalent to (13.8), if $\beta$ decreases, but the function $\lambda \mapsto \lambda \beta(\lambda)$ increases (which is a bit more than 13.6(a)). Indeed, then $(1+$ $\left.\frac{\lambda_{1}}{\lambda_{2}}\right) \frac{\beta\left(\lambda_{1}\right)}{\beta\left(\lambda_{2}\right)} \geq 1$ for all $\lambda_{1}, \lambda_{2}$ (check two cases, $\lambda_{1} \leq \lambda_{2}$ and $\lambda_{1} \geq \lambda_{2}$ ); taking $\lambda_{1}=n / \varepsilon_{n}$ and $\lambda_{2}=1 / \delta_{n}$ we get

$$
\frac{\varepsilon_{n}+n \delta_{n}}{\beta\left(1 / \delta_{n}\right)} \cdot \frac{\beta\left(n / \varepsilon_{n}\right)}{\varepsilon_{n}} \geq 1
$$

thus (13.8) implies (13.9).

Given $n_{1}<n_{2}<\ldots$, we may consider the sequence $\left(E_{n_{k}}\right)_{k=1}^{\infty}$ (where $E_{n}$ are given by (13.1) ) and ask whether it is infinitesimal, or not. Both Lemmas 13.6 and 13.7 (and their proofs) still hold when $n$ is substituted with $n_{k}$, and ' $n \rightarrow \infty$ ' with ' $k \rightarrow \infty$ '.

13.10 Lemma. Assume that $\nu_{1}, \nu_{2}$ are two measures, each satisfying 9.7(b) and (9.14); $\alpha, \beta:(0, \infty) \rightarrow(0, \infty) ; \alpha$ is a slowly varying function; $\beta$ decreases, but $\lambda \mapsto \lambda^{2} \beta(\lambda)$ increases; $\nu_{1}(d \lambda) \leq \alpha(\lambda) d \lambda ; \nu_{2}(d \lambda) \geq \beta(\lambda) d \lambda$; and $\lim \sup _{\lambda \rightarrow \infty}(\beta(\lambda) / \alpha(\lambda))=\infty$. Then there exists a sequence of elementary sets that satisfies (13.2), (13.3) w.r.t. $\nu_{2}$ but violates (13.3) w.r.t. $\nu_{1}$. 
Proof. We take $\lambda_{k} \uparrow \infty$ such that $\beta\left(\lambda_{k}\right) / \alpha\left(\lambda_{k}\right) \rightarrow \infty$. We note that $\lambda \alpha(\lambda) \rightarrow$ $\infty$; in particular, $\lambda_{k} \alpha\left(\lambda_{k}\right) \rightarrow \infty$. However, $\lambda_{k} \beta\left(\lambda_{k}\right) \gg \lambda_{k} \alpha\left(\lambda_{k}\right)$ (that is, $\left.\left(\lambda_{k} \beta\left(\lambda_{k}\right)\right) /\left(\lambda_{k} \alpha\left(\lambda_{k}\right)\right) \rightarrow \infty\right)$, and we can choose integers $n_{k} \uparrow \infty$ such that

$$
\lambda_{k} \alpha\left(\lambda_{k}\right) \ll n_{k} \ll \lambda_{k} \beta\left(\lambda_{k}\right) .
$$

Taking $\varepsilon_{k}=n_{k} / \lambda_{k}$ we have $\varepsilon_{k} \rightarrow 0$ and

$$
\frac{1}{\varepsilon_{k}} \alpha\left(\frac{n_{k}}{\varepsilon_{k}}\right) \rightarrow 0, \quad \frac{1}{\varepsilon_{k}} \beta\left(\frac{n_{k}}{\varepsilon_{k}}\right) \rightarrow \infty .
$$

The latter gives

$$
\frac{\varepsilon_{k}+2 n_{k} \delta_{k}}{\beta\left(\delta_{k}^{-1}\right)} \rightarrow 0 \quad \text { when } k \rightarrow \infty
$$

for some $\delta_{k}$ (namely, for $\delta_{k}=\varepsilon_{k} / n_{k}$, recall (13.8), (13.9)). Defining (recall (13.1))

$$
E_{k}=\bigcup_{l=0}^{n_{k}-1}\left(\frac{1}{n_{k}}\left(l+\frac{1-\varepsilon_{k}}{2}\right), \frac{1}{n_{k}}\left(l+\frac{1+\varepsilon_{k}}{2}\right)\right),
$$

we have mes $E_{k}=\varepsilon_{k}$ and mes $\left(\left(E_{k}\right)_{+\delta_{k}}\right)=\varepsilon_{k}+2 n_{k} \delta_{k}$. Lemma 13.6 (generalized to subsequences) states that $\left(E_{k}\right)_{k=1}^{\infty}$ satisfies (13.3) w.r.t. $\nu_{2}$. On the other hand, Lemma 13.7 (generalized to subsequences) shows that $\left(E_{k}\right)_{k=1}^{\infty}$ does not satisfy (13.3) w.r.t. $\nu_{1}$. It remains to note that (13.2) is ensured (w.r.t. $\nu_{2}$ ) by Lemma 13.4 .

The following result holds for both cases, real and complex.

13.11 Theorem. There is a continuum of mutually non-isomorphic product systems of type $I I I$.

Proof. For each $\alpha \in(0, \infty)$ we choose a positive $\sigma$-finite measure $\nu_{\alpha}$ on $[0, \infty)$ such that the function $\nu_{\alpha}(d \lambda) / d \lambda$ is strictly positive, continuously differentiable, and for $\lambda$ large enough, it is equal to $\ln ^{-\alpha} \lambda$ (recall (9.12)). Lemma 9.9 gives us the corresponding continuous sum of FHS spaces. As was said in Sect. 9, the continuous sum is homogeneous; Corollary 9.6 converts it into an HCP HS. By Lemma 10.3, it is of type III. The corresponding (by Theorem 1.9) product system is also of type III (see [A], Remark 3.3.3]). Functions $\lambda \mapsto \ln ^{-\alpha} \lambda$ are slowly varying, so, Lemma 13.10 and Theorem 11.3 show that these HCPs HS are mutually non-isomorphic. By Lemma 1.10, the corresponding product systems are mutually non-isomorphic. 


\section{References}

[Ac76] L. Accardi, On square roots of measures, In: Proc. Internat. School of Physics "Enrico Fermi", Course LX, North-Holland, 1976, pp. 167-189.

[AW66] H. Araki and E. J. Woods, Complete Boolean algebras of type I factors, Publications of the Research Institute for Mathematical Sciences, Kyoto Univ., Series A, 2 (1966), 157-242.

[A+] W. Arveson, Noncommutative Dynamics and E-semigroups, Manuscripts in Mathematics, Springer (to appear).

[A] W. Arveson, Four lectures on noncommutative dynamics, this volume.

[A99] W. Arveson, Semigroups of endomorphisms of $\mathcal{B}(H)$. Chapter 22 in: Lectures on Operator Theory (B.V.R. Bhat et al., eds.) Fields Institute Monographs. Amer. Math. Soc. 1999.

[A97] W. Arveson, Path spaces, continuous tensor products, and $E_{0^{-}}$ semigroups, Operator Algebras and Applications (A. Katavolos, ed.), Series C: Math. and Phys. Sci., vol. 495, Kluwer Academic Publishers, 1997, pp. 1-112. (Also arXiv:funct-an/9411006.)

[A96] W. Arveson, E $E_{0}$-semigroups in quantum field theory, Proc. Sympos. Pure Math. 59 (1996), 1-26.

[A89] W. Arveson, Continuous analogues of Fock space, Memoirs Amer. Math. Soc. 80 (1989), no. 3.

[Fe71] J. Feldman, Decomposable processes and continuous products of probability spaces, Journal of Functional Analysis 8 (1971), 1-51.

[Feller] W. Feller, An Introduction to Probability Theory and its Applications, Vol. II, second edition, Wiley, NY 1971.

[Ja] S. Janson, Gaussian Hilbert Spaces, Cambridge Univ. Press, 1997.

[OR] O.A. Oleŭnik, E.V. Radkevič, Second Order Equations with Nonnegative Characteristic Form, Amer. Math. Soc. and Plenum Press, 1973 (transl. from Russian).

[RY] D. Revuz, M. Yor, Continuous Martingales and Brownian Motion (second edition), Springer-Verlag, 1994.

[Ts+] B. Tsirelson, Scaling limit, noise, stability. In: Saint-Flour Summer School for Probability 2002; Lect. Notes in Math. (to appear). 
[Ts02] B. Tsirelson, Spectral densities describing off-white noises, Ann. Inst. H. Poincare, ser. B, 2002 (to appear).

[TV98] B.S. Tsirelson, A.M. Vershik, Examples of nonlinear continuous tensor products of measure spaces and non-Fock factorizations, Reviews in Mathematical Physics 10:1 (1998), 81-145.

\section{Index}

admissible norm, 42

asymptotically independent, 54

asymptotically orthogonal, 54

Boolean algebra of elementary sets, 12

$\mathcal{C}$, the space of closed sets, 15,24

complex versus real, 3, 17, 18, 42, 53, 57, 67

complexification

of real CP HS, 17

of real Hilbert space, 17

continuous sum of FHS spaces, 46

convergence

for admissible norms, 53

for Gaussian measures, 53

$\mathrm{CP} \mathrm{HS}=$ continuous product of Hilbert spaces, 4

CP HS over $[0,1], 12$

$\mathrm{CP} \mathrm{MC}=$ continuous product of measure classes, 20, 22

CP PS = continuous product of probability spaces, 23

$D$, countable set of discontinuity points, 15

decomposable

equivalence class of measures on $\mathcal{C}, 24$ probability measure on $\mathcal{C}, 26$

vector, 13

$E_{0}$-semigroup, 4

Effros $\sigma$-field, 24

elementary set, 12

embedded pair of CPs HS, 13

spectral measure on $\mathcal{C}, 16$

equivalence class of measures, 19

exponential of FHS space, 44

FHS equivalence operator, 42

equivalent norms, 42

sense, 46

space, 42

Fourier transform, normalized to be unitary, 48

$\Gamma$, action on exponential space, 44

Gaussian measure

on generalized functions, 49

over FHS space, 44

Hausdorff metric, 15

HCP HS = homogeneous continuous product of Hilbert spaces, 5

HCP $\mathrm{MC}=$ homogeneous continuous product of measure classes, 22

$\left(H / H^{\prime}\right)_{E}$, subspace, 14

Hilbert space: always separable, 3

history, 3

out of $\mathrm{HCP} \mathrm{HS}, 7$

homogeneous

continuous sum of FHS spaces, 47

CP HS, 5

CP MC, 22

independent

sub- $\sigma$-fields on a measure class, 21

sub- $\sigma$-fields on a probability space, 23

infinitesimal sequence of elementary sets,

13

and complexification, 17

isomorphism

and complexification, 17

local, of global HCPs HS, 10

of CPs HS, 5

over [0,1], 12

of CPs MC, 20, 22

of FHS spaces, 42

of measure classes, 19 
of probability spaces, 23

of product systems and local HCPs HS, 9

$L_{2}(X, \mathcal{B}, \mathcal{M})$, Hilbert space out of a measure class, 19

liminf for subspaces, 53

limsup for subspaces, 53

local CP HS, 9

measurable linear functional, 49

measure class, 19

measure space: always Lebesgue-Rokhlin, 19

negligible set, 19

noise

off-white, 49

white, 47

$\nu$, spectral measure on $[0, \infty), 48$

orthogonal FHS subspaces, 46

orthonormal basis of FHS space, 42

pair of embedded CPs HS, 13

perfect set, 27

portion of the random set, 35

probability space, 23

product system, 4

out of HCP HS, 7, 8

out of HCP MC, 22

out of HCP PS, 24

out of FHS spaces, 47

projection-valued measure, extension to, 15,16

$Q_{E}$, projection, 14

quasiorthogonal FHS subspaces, 46

quasiorthonormal basis of FHS space, 42 real versus complex, $3,17,18,42,53,57$, 67

regular additive function, 14

elementary set, 15

slowly varying, 64

smearing condition, 28, 29

spectral density (or measure) on $[0, \infty)$ of off-white noise, 49

spectral measure on $\mathcal{C}$, projection-valued and complexification, 18

of an embedded pair, 16

standard decomposable vector, 25

sub- $\sigma$-field, always completed, 21

time-reversed

CP HS, 36

CP MC, 36

HCP HS, 36

$\mathrm{HCP} \mathrm{MC}, 36$

topological sense, 46

type of CP HS, 13

$I I_{0}, 13$

$I I I, 13$

$U_{x}$, action on exponential space, 44

Vietoris topology, 15

$V_{y}$, action on exponential space, 44

$\xi_{x}$, linear random variable, 41,51

1, identity operator on a Hilbert space, 3

$\mathbf{1}$, indicator function of a set, 64

$\int f(t) \xi(t) d t$, linear random variable, 51

$\|\cdot\|_{\nu}$, shift-invariant norm, 48

$|\psi|^{2}$, measure, 23

BORIS TSIRELSON

School of Mathematics

Tel Aviv University

Tel Aviv 69978, Israel

tsirel@tau.ac.il

www.tau.ac.il/ tsirel/ 